\title{
High resolution differentiation of infectious agents at the level of antibody and nucleic acid by using peptide microarray and nanopore sequencing
}

\author{
Dissertation \\ to attain the doctoral degree (Dr. sc. agr.) \\ of the \\ Faculty of Agricultural Sciences Georg-August-Universität Göttingen
}

Submitted by

Sören Hansen

born on the 19/07/1989 in Niebüll 
1. Referee: Dr. Ahmed Abd El Wahed

2. Referee: Prof. Dr. Jens Tetens

3. Referee: Prof. Dr. Jürgen Hummel

Date of oral examination: 03.07.2019 


\section{List of Content}

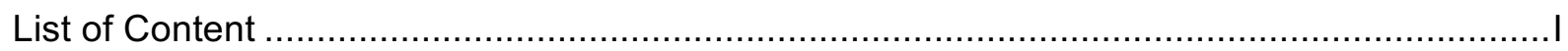

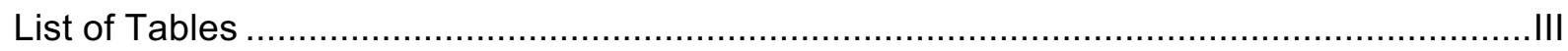

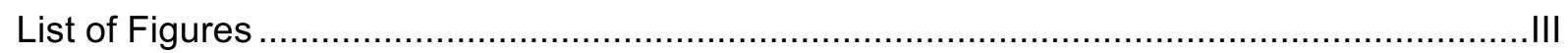

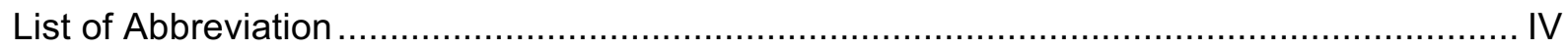

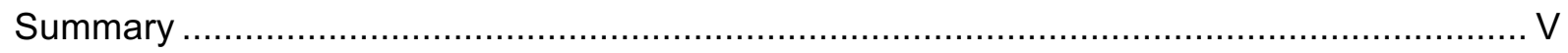

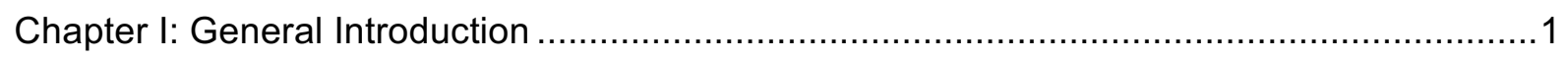

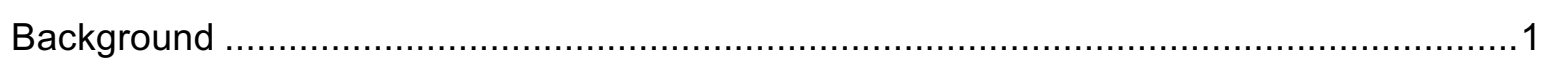

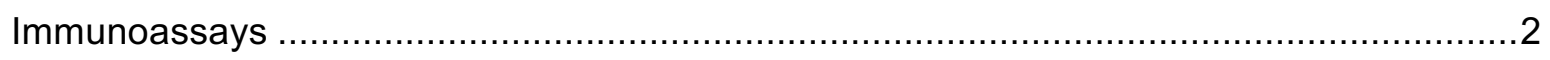

Lateral flow immunoassays for the detection of antigens or antibodies .........................

Methods for the identification of pathogens at the genomic level......................................4

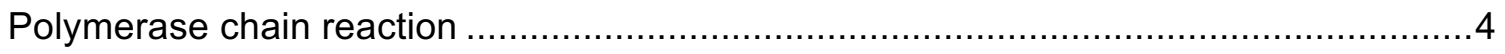

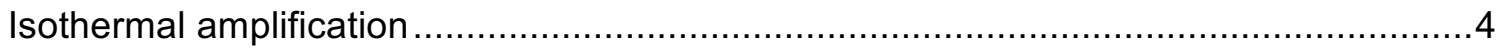

Equipment free nucleic acid amplification .......................................................

Metagenomic diagnostics as a tool for outbreak identification ................................. 8

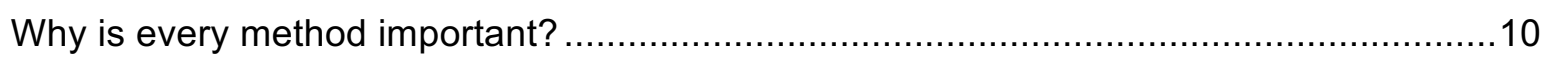

Solutions for mobile laboratories at point of care and point of need.............................. 10

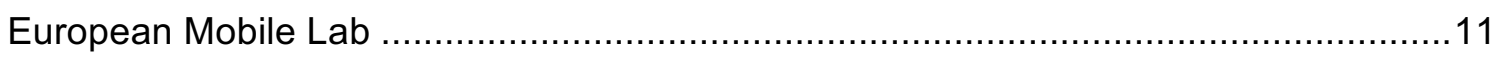

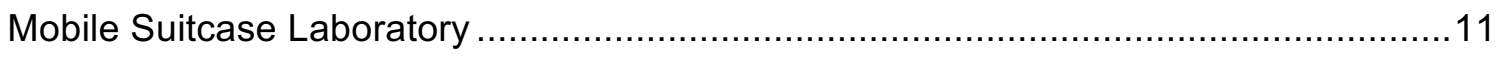

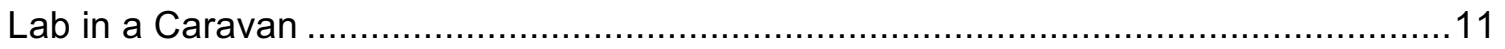

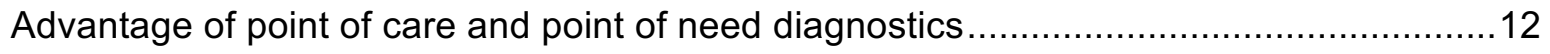

Barriers for the success of point of care and point of need tests.................................. 12

The need for capacity building at the link between human and animal health ..................13

The potential of point of care and point of need diagnostics in natural disasters ..............14

Why the importance of point of care and point of need testing increases ........................ 15

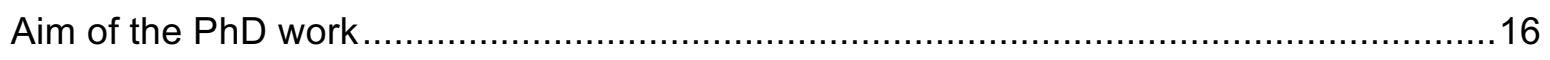

Chapter II: Development of Rapid Extraction Method of Mycobacterium avium subspecies paratuberculosis DNA from Bovine Stool Samples

Chapter III: Combination random isothermal amplification and nanopore sequencing for rapid identification of the causative agent of an outbreak

Chapter IV: Serotyping of foot-and-mouth disease virus using oxford nanopore sequencing33

Chapter V: Diagnosing Zika virus infection against a background of other flaviviruses:

Studies in high resolution serological analysis .... .38

Chapter VI: General Discussion 
Why Mycobacterium avium subspecies paratuberculosis, Zika virus and Foot and Mouth disease virus were selected as model pathogens in this $\mathrm{PhD}$ work....

The extraction of bacterial DNA in the field

Sequencing protocols for the rapid identification of the causative agent at outbreak situations .53

Differentiation of Zika virus to other flaviviruses using peptide microarrays .54

Conclusion.... 56

Chapter VII: References .57

Chapter VIII: Appendix 65

Supplementary Material........ Development of Rapid Extraction Method of Mycobacterium avium subspecies paratuberculosis DNA from Bovine Stool Samples .65 Supplementary Material. Serotyping of foot-and-mouth disease virus using oxford nanopore sequencing .68 Supplementary Material........Diagnosing Zika virus infection against a background of other flaviviruses: Studies in high resolution serological analysis .89

Author's contribution to the publications enclosed in this $\mathrm{PhD}$ thesis .99

List of Publications. 100

List of Presentations 101

Curriculum Vitae 103

Acknowledgements 104

Declarations 105 


\section{List of Tables}

Table 1: ASSURED criteria for Point of Care- and Point of Need test .................................. 1

Table 2: Different isothermal amplification techniques .....................................................

Table 3: Features of Loop-mediated Isothermal Amplification (LAMP) and Recombinase

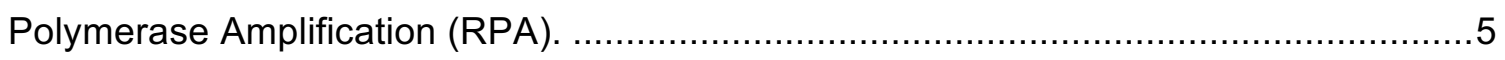

Table 4: Possibility of antigen/antibody detection in different types of infection....................10

Table 5: Important pathogens related with epidemic outbreaks after natural disasters as defined by Brock et al [95] in comparison to pathogens ......................................... 14

Table 6: Incubation times of selected important zoonotic and animal diseases...................15

\section{List of Figures}

Figure 1: Structure and type of a Lateral Flow Immunoassay..........................................

Figure 2: The Loop-mediated isothermal Amplification (LAMP) .................................... 6

Figure 3: The Recombinase Polymerase Amplification ................................................ 7

Figure 4: Detection of RPA amplicon in a lateral flow format. ....................................... 8

Figure 5: A: MinION sequencing device; B: Principle of nanopore sequencing. ...................

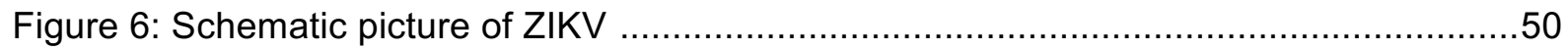

Figure 7: Phylogenetic relationship of selected flaviviruses ..........................................52 


\section{List of Abbreviation}

\begin{tabular}{|c|c|}
\hline ATR & antibody target region \\
\hline BSL & biosafety level \\
\hline CDC & United States Centers for Disease Control and Prevention \\
\hline cDNA & complementary DNA \\
\hline $\mathrm{cm}$ & centimetre \\
\hline DNA & deoxyribonucleic acid \\
\hline ELISA & enzyme-linked immunosorbent assay \\
\hline et al. & et alii \\
\hline FMDV & Foot and mouth disease virus \\
\hline g & gram \\
\hline HIV & human immunodeficiency viruses \\
\hline $\mathrm{kg}$ & kilogram \\
\hline LAMP & Loop-mediated isothermal Amplification assay \\
\hline LFIA & lateral flow immunoassay \\
\hline MAP & Mycobacterium avium subspecies paratuberculosis \\
\hline $\mathrm{mg}$ & milligram \\
\hline $\mathrm{ml}$ & millilitre \\
\hline NALFA & Nucleic Acid Lateral Flow assay \\
\hline NALFIA & Nucleic Acid Lateral Flow Immunoassay \\
\hline NS & non-structural protein \\
\hline OIE & World Organization for Animal Health \\
\hline ONT & Oxford Nanopore Technologies \\
\hline PCR & Polymerase Chain Reaction \\
\hline Ph.D & Doctor of Philosophy \\
\hline POC & Point of Care \\
\hline POCT & Point of Care test \\
\hline PON & Point of Need \\
\hline PONT & Point of Need test \\
\hline RNA & ribonucleic acid \\
\hline RPA & recombinase polymerase amplification \\
\hline RT-PCR & reverse-transcription polymerase chain reaction \\
\hline ss & single-stranded \\
\hline THF & tetrahydrofuran \\
\hline WHO & World Health Organization \\
\hline ZEBRA & Zika in Brazil Real Time Analysis \\
\hline$\mu l$ & microlitre \\
\hline
\end{tabular}




\section{Summary}

In recent years, Point of Care (POCT) or Point of Need tests (PONT) have been established in order to provide accurate and rapid diagnostics directly at field level or at site of outbreaks. These assays can help decision makers to take the right action without delay. Typically, POCT and PONT rely on the genomic identification of pathogens or tracking its immunological fingerprint. Recognition of a pathogen's genome in the field using molecular assays highly depends on the efficiency of nucleic acid extraction. Moreover, the sensitivity and specificity of molecular assays rely on previous knowledge of DNA or RNA sequences.

Classical immunological based tests i. e. whole antigen based ELISA lack specificity due to high antigen homology within the respective genus of pathogen as seen, for instance, in flaviviruses.

To address these drawbacks, this $\mathrm{PhD}$ work applied three different approaches: a rapid nucleic acid extraction method, metagenomic diagnostics and peptide microarray.

During the course of this work a protocol for the rapid extraction of nucleic acids of Gram-positive bacteria was developed employing magnetic bead based reverse purification technology. The extraction was carried out within 20 minutes. In combination with the recombinase polymerase amplification assay, a $90.9 \%$ clinical sensitivity and $100 \%$ clinical specificity were reached.

A protocol for the rapid and field applicable identification of RNA viruses to determine the causative agent in an outbreak by diagnostic metagenomics was implemented. Therefore, multiple displacement isothermal amplification and nanopore sequencing were combined to test a mock sample containing Zika virus (ZIKV). The whole procedure was conducted in less than seven hours including sample preparation. Presence of ZIKV sequences within the produced reads was determined by offline BLAST search. The procedure was further developed for rapid serotyping of foot and mouth disease virus (FMDV) relying on nanopore sequencing and offline BLAST search. The offline BLAST search was highly successful in categorizing seven FMDV serotypes by using the $\mathrm{P} 1$ region (specificity: $98.3 \%$ ) instead of whole genome (24.8\%), P2 (23.6\%) or P3 (21.4\%) regions.

A peptide microarray was used to identify a bundle of Zika Virus (ZIKV) specific antibody targets for the differentiation of other flaviviruses. Briefly, a total of 1643 overlapping oligopeptides were synthesized and printed onto glass slides. The oligopeptides cover the whole polyproteins of ZIKV originated from Africa, Brazil, USA 
and French Polynesia. The ZIKV scanning microarray chips were applied to examine three human serum pools from Zika outbreaks in Senegal and Cape Verde, in Brazil, and from overseas travellers returning to the EU as well as a pool of well described sera from patients that had suffered from dengue, yellow fever, tick-borne encephalitis or West Nile virus infections. Altogether, sixty-eight antibody target regions were identified, most of them previously unknown, from which thirteen could be classified as potentially ZIKV specific.

While the fast and sensitive extraction of DNA in the field was shown to enable genomic assays at PON, the two sequencing protocols have the potential to provide field applicable metagenomic diagnostics at point of need as workflows were performed in a mobile suitcase laboratory. The results obtained by the peptide microarray can be seen as a founding set of analytical tools for serological discrimination of ZIKV from other flaviviruses.

In conclusion, this PhD study paves the way for reliable POCT and PONT and represents an example of research at One Health level, in which the health of human, animal and environment matter. 


\section{Chapter I: General Introduction}

\section{Background}

Many different microorganisms such as bacteria, viruses, fungi or parasites are existing in the environment. Most of them are important to maintain the ecosystem on earth, however, some of them are pathogenic and cause infectious diseases in both human and animals. Diseases that are transmitted from animal to humans are called zoonotic [1]. Infectious diseases are one of the major reasons for death especially in the low and middle-income regions of the world. In addition, there are high economic losses due to diseased persons or animals [2, 3]. The World Organization for Animal Health (OIE) estimates the average economic losses in animals are around 20 percent [4]. A profound surveillance system is one of the main factors to prevent the spread of infectious diseases [5] which can only be archived with highly advanced diagnostics. The current diagnostic approaches rely on centralised reference laboratories with high throughput, due to the need of complex and expensive devices. Point-of-Care testing (POCT) describes the identification of pathogens near the patient with a fast turnaround time and the potential to immediately change in the health management [6]. While the term POCT is used for human patients and samples, Point-of-Need testing (PONT) has a broader meaning including also on-site testing of environment, animals, and food samples, although this term is not clearly defined yet. The World Health Organisation (WHO) formulated the characteristics of POCT and PONT as affordable, sensitive, specific, user friendly, robust and rapid, equipment free, and deliverable to those who need them (ASSURED criteria, Table 1) [7].

Table 1: ASSURED criteria for Point of Care- and Point of Need test as formulate by the WHO [7].

\begin{tabular}{c|l} 
A & Affordable \\
\hline$S$ & Sensitive \\
\hline$S$ & Specific \\
\hline U & User-friendly \\
\hline R & Robust and rapid \\
\hline E & Equipment-free \\
\hline D & Deliverable to those who need them
\end{tabular}


Examples for rapid diagnostics in the field are the POCTs for malaria and human immunodeficiency viruses (HIV) as well as the PONTs for Bluetongue virus [8], Schmallenberg virus [9], Bovine diarrhoea virus [9], Foot and Mouth disease virus (FMDV) [10], and the Lumpy Skin Disease Virus [11]. As a consequence, disease control as well as change in treatment and care was achieved [12,13] particularly, in regions where stable electricity supply, highly trained personal or specialized devices are not available [12]. Most POCT and PONT rely on immuno-techniques to detect antigens or antibodies, but methods for the identification of the pathogen at molecular level are on the rise [14]. Details about these methods are mentioned below.

\section{Immunoassays}

Immunoassays are based on binding of an antibody and an antigen to each other. For detection, the antigen is immobilized on a solid phase and bound to a specific primary antibody which is recognized by a secondary labelled antibody. This is named indirect immunoassay [15]. In a competitive assay, the target molecule competes with labelled antigens or antibodies in binding to the immobilized molecules. Another method is the so-called sandwich assay. Here, the target antigen is fixed between two of the same immobilization and identification molecules. [16].

Labelling of the secondary antibodies is accomplished for instance by enzymes (in case of enzyme-linked immunosorbent assay, ELISA), gold nanoparticles or silver nanoparticles lateral flow immunoassays (LFIA, [17]). ELISA has long and complex protocols or requires highly sophisticated laboratory equipment, while the most employed technique for POC and PON applications is the LFIA.

\section{Lateral flow immunoassays for the detection of antigens or antibodies}

The first LFIA were brought into the market in the early 1980s, since then, many tests have been developed [18]. LFIA is able to detect pathogen specific antigens and/or antibodies. Lateral flow assays are among the simplest equipment-free methods as they are performed in a small disposable cartridge (Figure 1). LFIA do not require pipetting, washing steps and can be performed by untrained personal. In addition, no cold chain is required [19]. The results can be obtained in 10-30 minutes. 


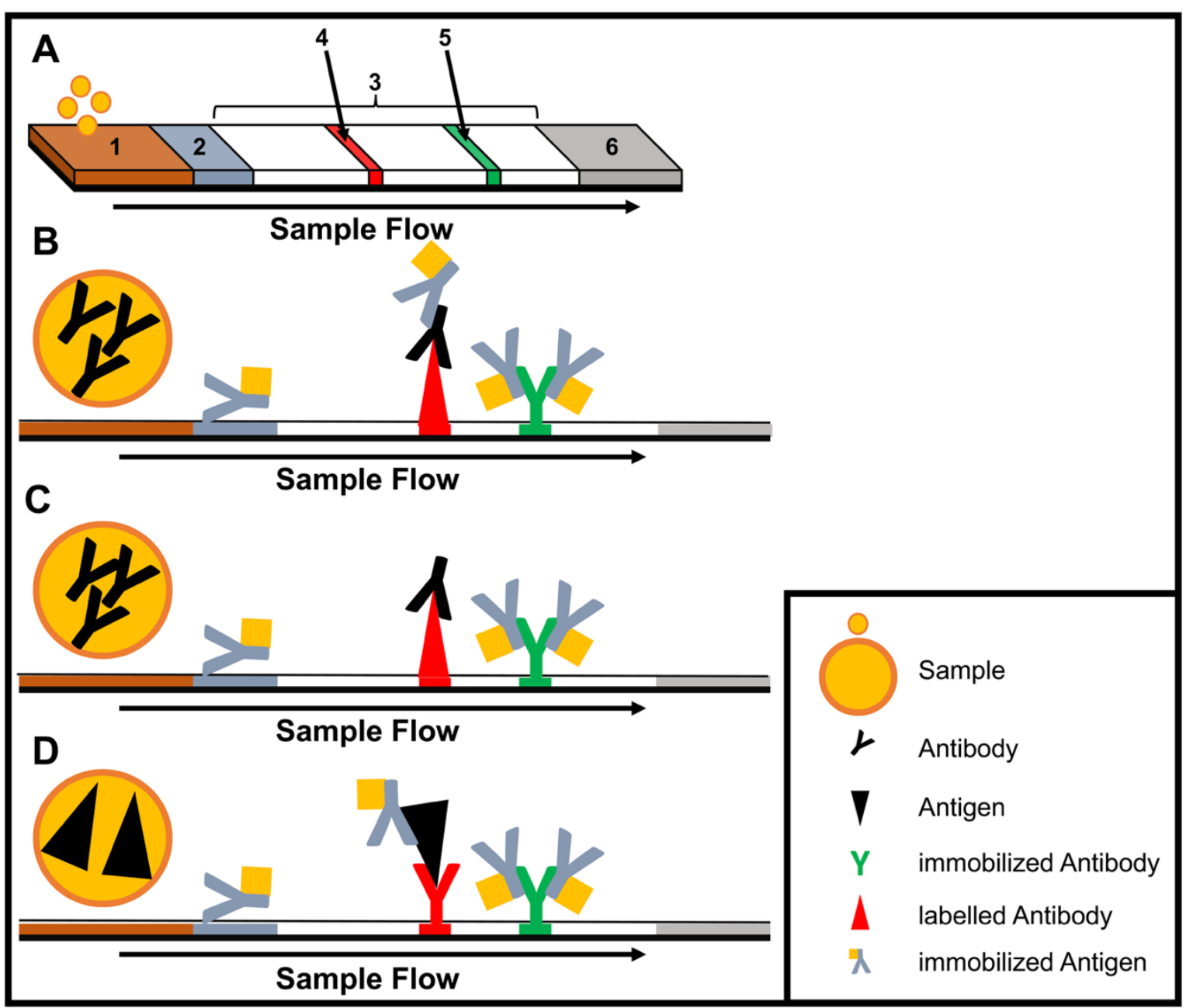

Figure 1: Structure and type of a Lateral Flow Immunoassay. (Panel A) schematic design: 1, sample pad; 2, conjugate pad; 3, membrane; 4, test line; 5, control line; 6, adsorbent pad. (Panel B) indirect lateral flow immunoassay. (Panel C) competitive lateral flow immunoassay (Panel D) sandwich lateral flow immunoassay.

The principle of a LFIA is as follows: the sample is brought onto the sample pad and flows in the opposite direction by adsorption. While the sample passes the conjugate pad, a labelled target specific antibody binds to the target molecule in the sample (antigen or antibody). Afterwards, the labelled antibody-target complex is immobilized to the membrane by a specific capture molecule (antibody or antigen) adhered to the membrane at the test line. The unbound labelled antibodies are captured at a control line by immobilized antibodies. In case of a positive sample, the accumulation of the labelled antibodies leads to a colouration of both test lines. In case of a negative sample only the control line is coloured. The adsorbent pad takes up the excess liquid $[20,21]$. In case of pathogens or targets, which are not suitable for the indirect or sandwich designed LFIA, a competitive layout can be applied. This results in only one coloured test line in case of a positive test [20].

Recently, multiplex LFIA have been developed detecting multiple targets in a single test $[22,23]$. Paper-based micro fluid devices ( $\mu$ PAD) are yet another development of 
the lateral flow technique. Here, the sample is guided by hydrophobic channels [24]. This design enables more sophisticated applications like the combination of multiple serological assays in a single device [25-27].

Sensitivity and specificity of LFIA highly depend on labelling and binding affinity of the tested biomolecules [17, 28]. A high concentration of molecules is necessary to achieve positive results. Therefore, LFIA will produce a negative result, when testing samples containing only few target molecules. Moreover, in some occasions sample preparation is necessary since the test design only allows liquid non-viscous samples [29]. Quantification of targets is not possible using the LFIA.

\section{Methods for the identification of pathogens at the genomic level}

Nucleic acid amplification methods have the advantage of being highly sensitive as opposed to immunological assays due to the amplification step. DNA is amplified using with cycling methods such as Polymerase Chain reaction (PCR) or isothermal amplification.

\section{$\underline{\text { Polymerase chain reaction }}$}

The Polymerase Chain Reaction (PCR) as the first molecular assay was developed by Kary B. Mullis in 1983 [30]. This method utilizes two oligonucleotides (primers) each complementary to one of the target DNA strands spanning the amplified region. The primers act as trigger for the synthesis of the complementary strands by a polymerase. Amplification of the target sequence is promoted by different heat steps. In the denaturation step $\left(95^{\circ} \mathrm{C}\right)$, the double strand of the target DNA is separated. In the annealing step $\left(50-75^{\circ} \mathrm{C}\right)$, the primers hybridize to the target region in the single strand DNA. Final extension takes place using the polymerase at $72-78^{\circ} \mathrm{C}$. Together, the three steps form a cycle. A single PCR run consists out of 30-40 cycles [31]. Real-time detection of amplification is mostly achieved with a $5^{\prime}$ fluorophore and $3^{\prime}$ quencher labelled probe complementary to the target sequence [32].

\section{$\underline{\text { Isothermal amplification }}$}

Isothermal amplification assays have the advantage of employing a constant reaction temperature for the amplification. This offers more utility in the field due to the use of portable heat sources and shorter run time in comparison to the cycle driven PCR [33, 
34]. Moreover, most isothermal methods are known to be resistant to inhibitors existing in complex samples like blood [35]. Several different methods for isothermal amplification have been developed in the past years (Table 2). From these methods, the two most evolving techniques are the Loop-mediated Isothermal Amplification (LAMP, Figure 2) and the Recombinase Polymerase Amplification (RPA, Figure 3). The features of both methods are shown in Table 3.

Table 2: Different isothermal amplification techniques

\begin{tabular}{|c|cccc|}
\hline Method & $\begin{array}{c}\text { Reaction } \\
\text { temperature }\left({ }^{\circ} \mathbf{C}\right)\end{array}$ & $\begin{array}{c}\text { Time to } \\
\text { result (min) }\end{array}$ & $\begin{array}{c}\text { No. of } \\
\text { primers }\end{array}$ & Probe \\
\hline $\begin{array}{c}\text { Helicase-Dependent } \\
\text { Amplification (HDA) } \\
\text { Rolling Circle Amplification } \\
\text { (RCA) }\end{array}$ & 37 & 60 & 2 & - \\
$\begin{array}{c}\text { Recombinase Polymerase } \\
\text { Amplification (RPA) }\end{array}$ & 37 & 90 & 1,2 or $>2$ & $+/-$ \\
$\begin{array}{c}\text { Nucleic Acid Sequence } \\
\text { Based Amplification } \\
\text { (NASBA) }\end{array}$ & $49-42$ & $3-10$ & 2 & + \\
$\begin{array}{c}\text { Nicking Enzyme } \\
\text { Amplification Reaction } \\
\text { (NEAR) }\end{array}$ & 60 & $90-120$ & 2 & + \\
$\begin{array}{c}\text { Loop-mediated isothermal } \\
\text { Amplification (LAMP) }\end{array}$ & $60-65$ & $2-5$ & 2 & $+/-$ \\
\hline
\end{tabular}

+ use of probe for real-time detection possible $\quad$ +/- use of probe possible, but usually not applied - use of probe not possible

Table 3: Features of Loop-mediated Isothermal Amplification (LAMP) and Recombinase Polymerase Amplification (RPA).

\begin{tabular}{|l|c|c|}
\hline \multicolumn{1}{|c|}{ Feature } & LAMP & RPA \\
\hline isothermal & $\checkmark$ & $\checkmark$ \\
\hline visual read-out & $\checkmark$ & $\checkmark$ \\
\hline portable heat source & $\checkmark$ & $\checkmark$ \\
\hline easy to implement in field applications & & $\checkmark$ \\
\hline fast result & & $\checkmark$ \\
\hline pair of primers & & $\checkmark$ \\
\hline simple assay design & & $\checkmark$ \\
\hline highly resistant to inhibitors & & $\checkmark$ \\
\hline long storage of reagents at room temperature & & \\
\hline
\end{tabular}




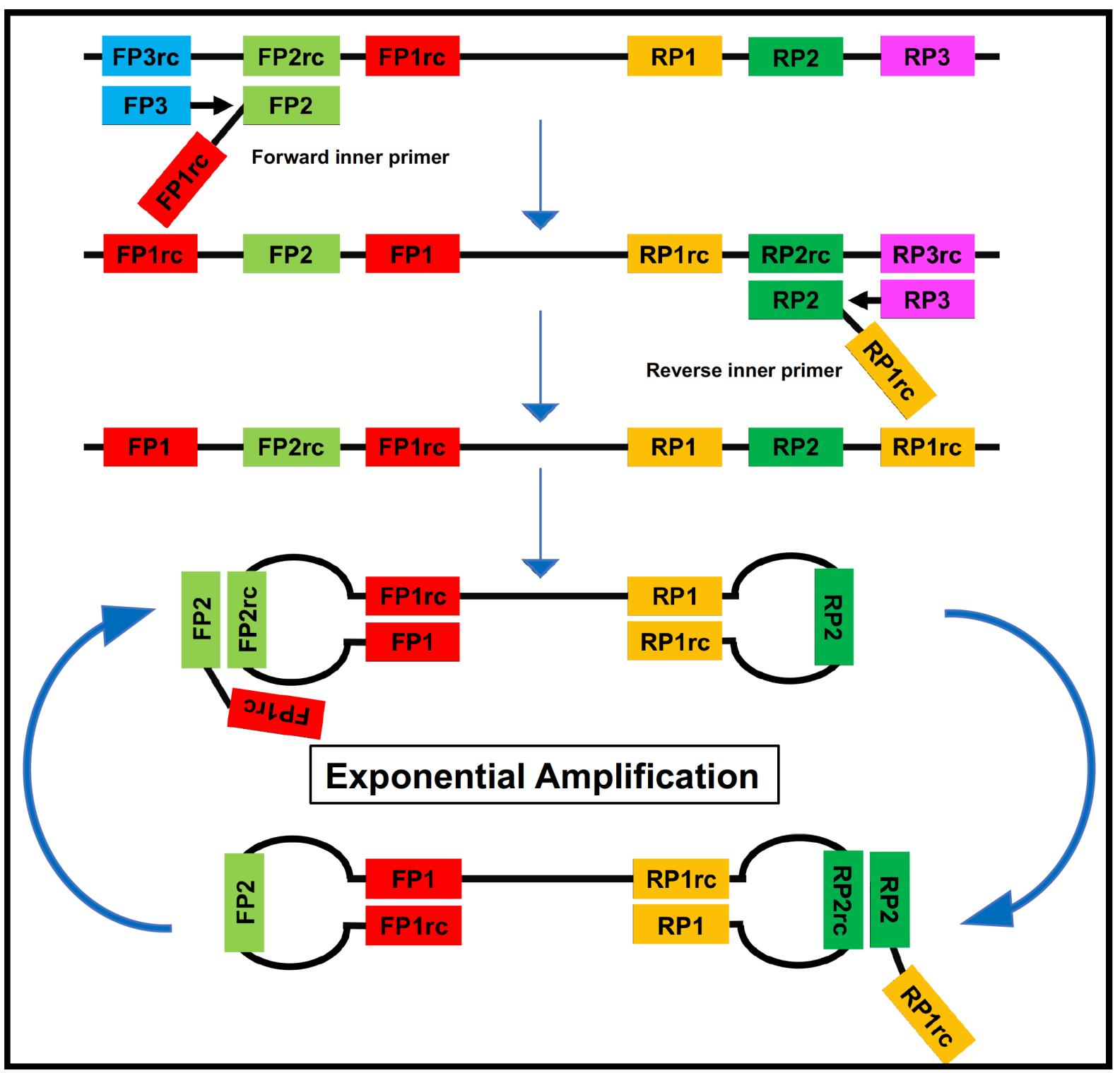

Figure 2: The Loop-mediated isothermal Amplification (LAMP) is based on a so called auto-cycling strand displacement DNA synthesis using a strand displacing DNA polymerase and two pairs of target specific primers. The inner primers contain two sequences each corresponding to the sense and anti-sense ( $\mathrm{rc}$ ) strand of the target DNA, respectively, linked with a TTTT-spacer. The inner primers initiate a complementary synthesis of the target DNA while the outer primers (FP1 and RP3) initiate a strand-displacement synthesis resulting in the release singlestranded DNA linked by inner primers. The single-stranded DNA forms stem-loops by self-annealing of the corresponding sequences and acts as template for exponential amplification [36]. 


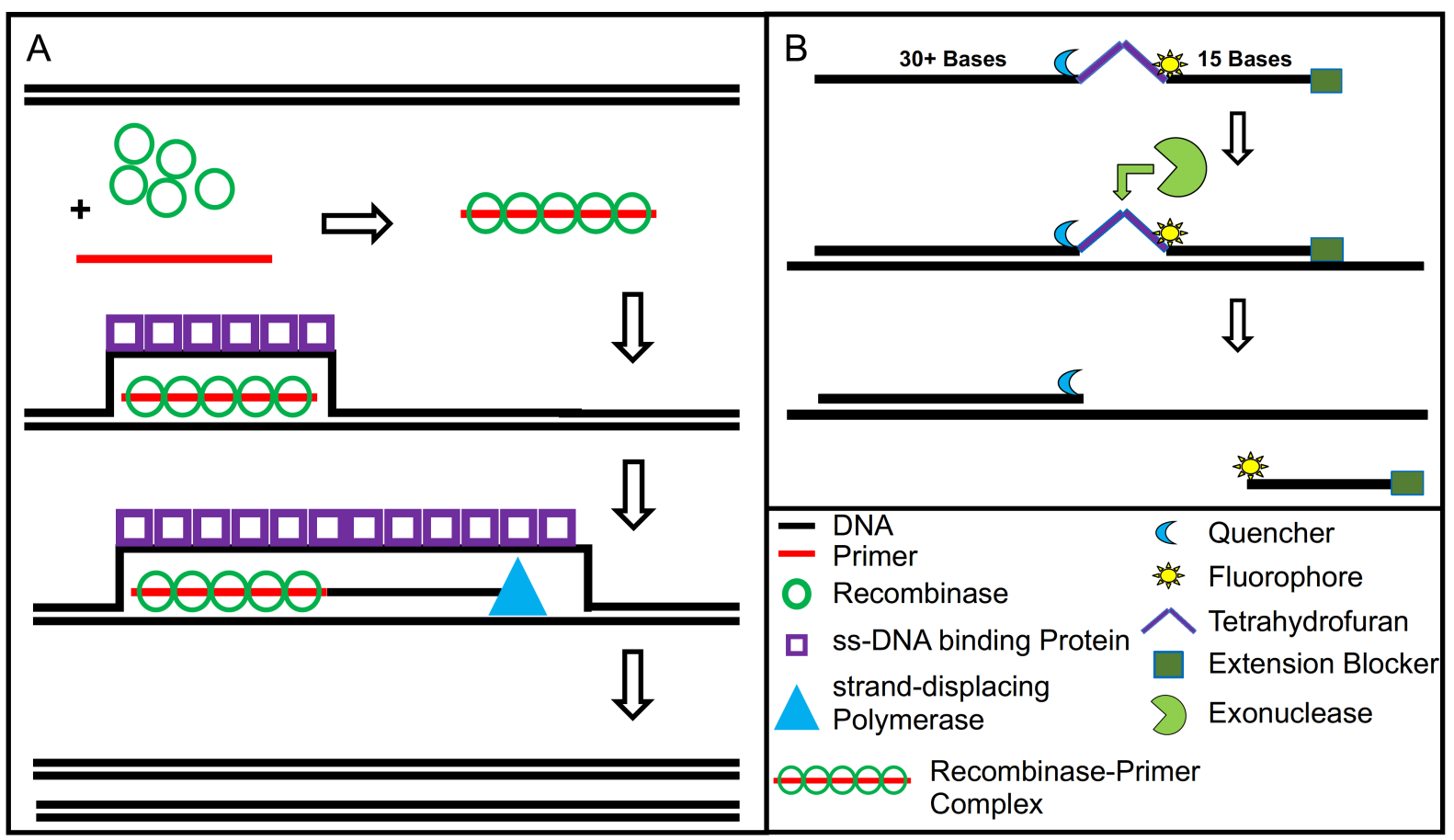

Figure 3: The Recombinase Polymerase Amplification (A) employs a recombinase enzyme, a single-stranded binding protein, a strand-displacing (ss-DNA) polymerase, and a pair of target specific primers. The primers form together with the recombinase enzyme the Recombinase-Primer complex which allows them to invade the doublestranded helix of the target DNA at the respective recognition site. The strand-displacing polymerase elongates the primers while the ss-DNA binding protein stabilized the displaced strand in order to prevent self-annealing and ejection of the primers by branch migration. Real-time detection of amplification (B) is achieved by a sequence specific probe consisting out of a tetrahydrofuran abasic-site mimic (THF) flanked by fluorophore and quencher labelled nucleotides as well as an extension blocker at the $3^{\prime}$-end. As the probe pairs with the complimentary sequence, a double-strand specific exonuclease slices the THF and the fluorophore is dissociated resulting in a signal [37].

\section{Equipment free nucleic acid amplification}

The implementation of equipment free nucleic acid amplification at POC and PON is extremely challenging [38]. Even LAMP assays [38-40] are still in need of a heating source. Nevertheless, to overcome the required power supply for LAMP reaction, LaBarre et al. developed a portable heating cartridge based on exothermal-reaction and engineered phase-change material. Heat is generated by the reaction of calcium oxide and water. To keep the temperature in the optimal range, a fat-based compound with a specific heat capacity and melting point $\left(65^{\circ} \mathrm{C}\right)$ is applied. Beside LAMP, this cartridge is also suitable for other isothermal reaction like RPA or the Nicking Enzyme Amplification Reaction (NEAR [41, 42]).

While in LAMP assays nucleic amplification results in a turbid reaction mix, the equipment free detection of amplification remains challenging in other isothermal techniques. However, since LAMP relies on multiple primers, an unspecific amplification can happen, probably due to cis and trans priming among the single primers. Therefore, specific amplicon detection is also necessary [43]. 
To provide a simple and inexpensive read-out of the different amplification methods, nucleic acid lateral flow (immuno-) assays (NALFA/NALFIA) were applied [44]. These assays are mainly based on the capture of tagged amplicons or detection of amplicons in a sandwich-format [29]. The use of lateral flow technique liberates from the need of sophisticated devices (e.g. fluorescence reader). In some studies, isothermal amplifications were shown to operate on paper-based devices. While paper-based RCA [45] works on room temperature without equipment, RPA was also conducted equipment-free using the body heat for incubation combined with a lateral flow-based detection of the tagged amplicons. The principle of RPA lateral flow detection is shown in Figure 4, [46-48]).

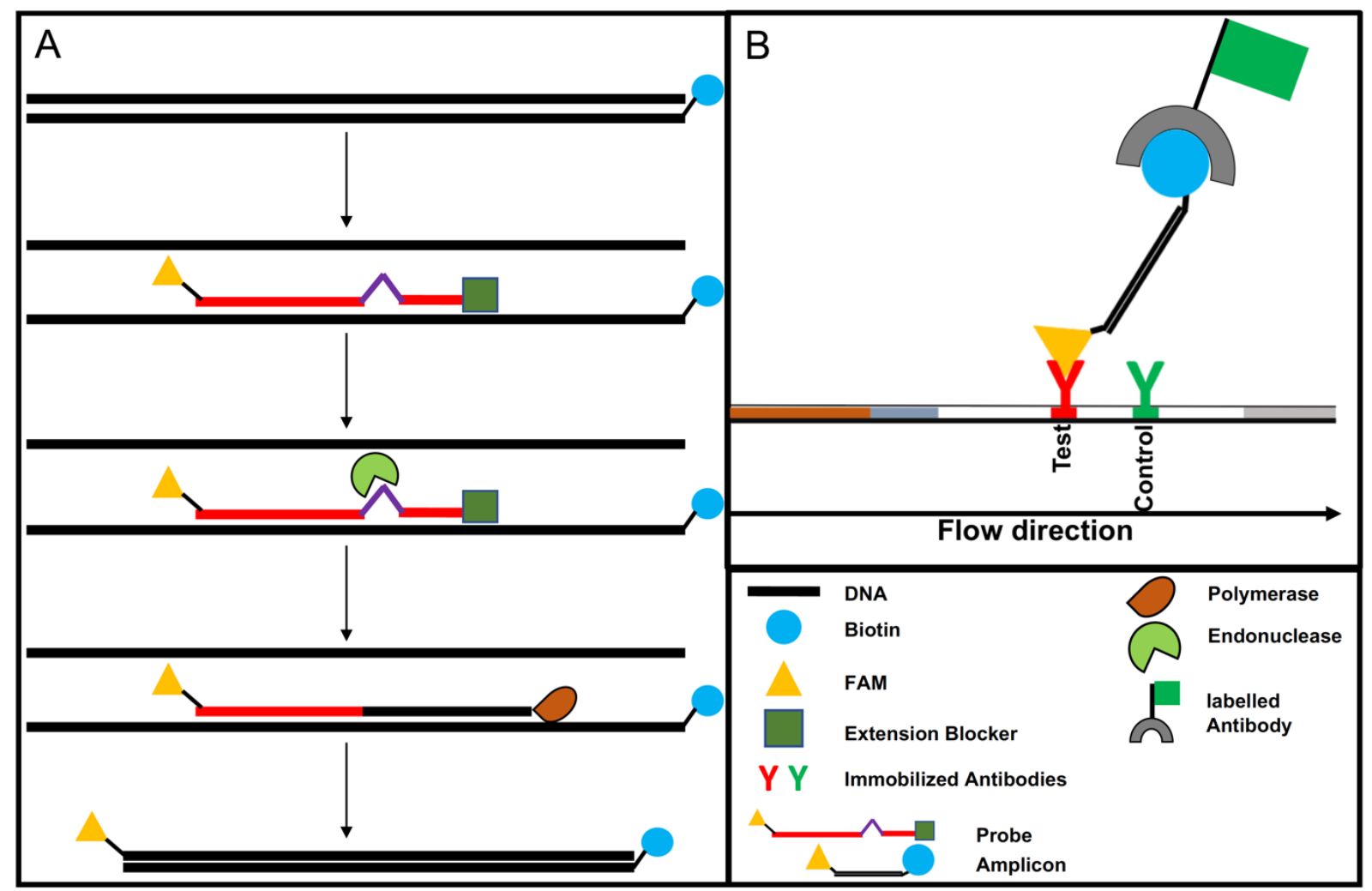

Figure 4: Detection of RPA amplicon in a lateral flow format. The use of a 5 end Biotin labelled reverse primer in the RPA reaction leads to an antigenic tagged reverse strand. When the 5'FAM-labbeled probe binds to the complimentary strand a double-strand specific endonuclease slices the THF. The extension blocker is released and the remaining oligonucleotide can act as primer resulting in a FAM and Biotin labelled amplicon (A), which can be detected in a lateral flow sandwich format $(B,[49])$.

\section{Metagenomic diagnostics as a tool for outbreak identification}

Metagenomic diagnostics is the identification of pathogens by metagenomic information obtained from nucleic acid sequencing [50]. This approach is based on the sequencing and identification of either all the nucleic acids present in the sample (shotgun metagenomics) or a particular group of previously generated amplicons (targeted-amplicon sequencing) [51]. 
The molecular detection through PCR or isothermal amplification of a pathogen requires a known target nucleic acid sequence. Thus, in an outbreak of unknown cause, selection of the right molecular assay upon the clinical signs is challenging and a novel pathogen or variants of known infectious agent may remain undetected. Metagenomic diagnostics overcomes this problem by sequencing and identifying all of the nucleic acids within the sample and thereby preparing for simpler diagnostic testing. Also, information about epidemiology and transmission route can be achieved [52]. However, the performance of this method strongly relies on the used genome database and depth of sequence analysis as well as on the amount of generated reads [53]. The major drawback of meta genomic diagnostics are the high costs per sequencing sample and the relatively long turnover times [52].

Sequencing methods require heavy and complex devices. However, the introduction of nanopore sequencing to the market by Oxford Nanopore Technologies (ONT, Oxford, UK) allows third-generation sequencing at point of need due to small portable sequencing devices (Figure 5, [54]). The great utility of nanopore sequencing at point of need was proven by identification of pathogens direct from clinical and tick samples $[55,56]$ as well as during the surveillance of Ebola, Lassa, Yellow Fever, and Zika outbreaks in West-Africa and South-America [57-60].

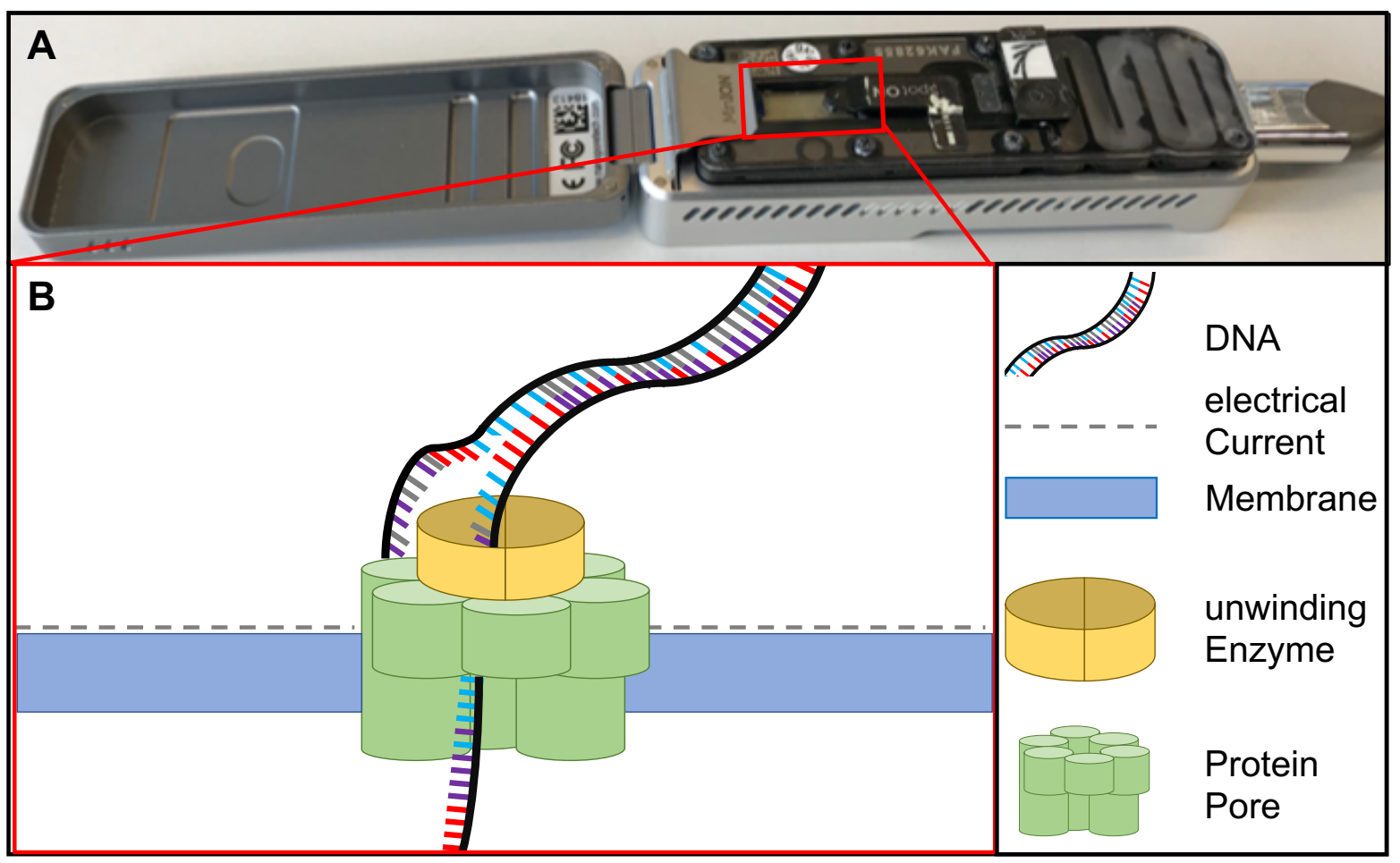

Figure 5: A: MinION sequencing device (ONT, Oxford, UK); B: Principle of nanopore sequencing. A protein pore is embedded in a membrane set under a certain electrical current. As the DNA molecule passes through the pore each nucleotide is recognized by the individual change caused in the voltage. 


\section{Why is every method important?}

Immunoassays are an inexpensive, robust, and simple way to use for the detection of antibodies. Since different types of antibodies can be detectable several days or up to years in the blood [61], the immunoassays represent an easy way for surveillance of previous and ongoing chronic infection. However, the identification of a disease based solely on antibody detection may lead to a false assumption about the diseased status of the individual. During persistent infections a detection of antigen and antibody is normally possible over a long period of time, a latent infection (as a special case of persistent infections) is characterized by a very low antigen concentrations but high antibody level (for definitions see Table 4, [62]). In the latter case, nucleic acid amplification assays are needed as they can detect a low number of genomes with high specificity, which is especially helpful as for some chronic infections, the concentration of pathogens, antigens and antibodies varies over the time [63]. Thus, the combination of immunological and genomic methods is necessary. On the other hand, as the immune response towards a disease starts a few days post infection and reaches the highest point around two weeks post infection [64], the detection of an acute infection is not possible solely based on antibodies. When the pathogen exists with a low concentration in the sample or is detectable for a short period of time (like swine influenza virus [65]), nucleic acid amplification assays are a good tool for diagnosis of such diseases.

Table 4: Possibility of Antigen/Antibody detection in different types of infection [62].

\begin{tabular}{|c|c|c|}
\hline Type of infection & Detection of antigens & Detection of antibodies \\
\hline acute & + & - \\
\hline persistent & + & + \\
\hline latent & - & $+/-$ \\
\hline chronic & $+/-$ & + \\
\hline
\end{tabular}

+ detection possible, - detection not possible, +/- detection temporarily possible

\section{Solutions for mobile laboratories at point of care and point of need}

The need of on-site diagnostic facilities was demonstrated during the West African Ebola outbreak as health systems of the affected countries were overloaded or not existing. Mobile laboratories were developed to enable diagnosis during outbreak 
situations. Their great advantage is the capacity of performing several diagnostic tests just as well as a central laboratory while being in the field at the site of an outbreak.

\section{European Mobile Lab}

To overcome the problem of long distances to the central diagnostic laboratories during the haemorrhagic fever outbreak in West-Africa, the European Mobile Lab Project established a moving laboratory unit in Nigeria, consisting of 27 boxes, each of them approximately $20 \mathrm{~kg}$ to $30 \mathrm{~kg}$ in weight. These boxes contain more than 400 equipment items needed to set up a fully functional BSL3 or BSL4 diagnostic laboratory in a tent or in a local house. Minimum requirements are at least 28 square meters of space and a car for constant energy supply [66].

\section{Mobile Suitcase Laboratory}

Originally developed for the field applicable detection of avian influenza, the mobile suitcase laboratory was implemented in Guinea during the time of the West-African Ebola outbreak. This PON diagnostics solution consists of two trolley cases, a solar panel, a power pack, and an optional glove box. One of the trolley cases is employed for nucleic acid extraction as all instruments needed are fixated in the suitcase. The second trolley case contains all instruments for the detection of the extracted nucleic acid via RPA. The devices are placed on foam covered by a PVC top layer to be protected from shocks and to facilitate disinfection. The glove box can be used for sample disinfection and nucleic acid extraction in case of BSL3 and BSL4 pathogens. All materials, kits, and reagents needed for nucleic acid extraction and isothermal amplification are stored in the suitcases. Due to the compact design, the mobile suitcase laboratory is easy to operate, also in challenging surroundings [67-69].

\section{$\underline{\text { Lab in a Caravan }}$}

Another example for a mobile lab solution is the lab in a caravan. Based on the experiences made during the Ebola outbreak in West-Africain 2016, the Zika in Brazil Real Time Analysis (ZEBRA) project implemented two labs in caravan. These complete BSL 3 laboratories were set up in a trailer and used to collect and sequence samples of ZIKV along parts of the Brazilian east coast [70]. 
The Praesens Foundation developed a mobile laboratory implemented in a small truck [71]. The mobile laboratory contains a real-time thermocycler and a BSL3 glove box for sample preparation. The laboratory is air conditioned and can be hermetically sealed by the inside lower air pressure. For communication, Wi-Fi, mobile, and satellite connection are implemented. The lab has two batteries for power supply lasting more than 72 hours [72].

\section{Advantage of point of care and point of need diagnostics}

The big advantage of POC and PON diagnostics is the identification of pathogens directly at the site of the diseased person or animal with small turnaround times. So, the persons responsible for treatment can react more quickly according to the result [73]. The ease of international travel and trade are the reasons for global spread of many infectious diseases, the use of POCT and PONT at airports, harbours, and quarantine stations can prevent the entry of diseases into a country. Furthermore, the sample volume needed for the tests is often smaller than in conventional laboratory tests [74]. In addition, the use of POCT and PONT can lead to more adherence and optimisation of the treatment regimen at the clinical side. Economically, it reduces cost for applying unspecific treatment, laboratory equipment, and staff [75]. Routine use of POCT and PONT can support the building of broad and comprehensive diagnostic infrastructure, especially in low resource settings, as the tests are mostly equipment free and only small investments in local facilities are necessary [76].

\section{Barriers for the success of point of care and point of need tests}

The barriers of success of POCT and PONT often depend on several factors: political, regulatory, cultural, economic, or communication and perception [77, 78]. Rasmussen et al. found in Guinea a significant negative correlation between the willingness to be tested for pathogens and the level of education as well as the stability of the political system [79]. In addition, Engel et al. showed that barriers differ between cultures. In India, people must go to the diagnostic laboratories by themselves, provide samples or receive results there and share them with the person responsible for treatment afterwards. This may lead to mistakes in communication of the results since the main responsibility is with the patient him or herself, as private and untrained person. In contrast, the diagnostics in South Africa are highly centralized. Most mistakes occur due to bad communication between involved facilities or persons (misspelled names 
or lost results), sample transportation problems, breakdown of computer systems, and others. All these factors lead to long turn-around times in the diagnostics [80]. Normally, prices of tests decrease by time once the tests have entered a broad market and in accordance the economic concerns of the users. Nevertheless, other doubts like implementation of the test into routine testing, the ease of use, supply, and storage stability of the test kits especially in rural areas remain big challenges [81, 82]. Additionally, decision maker fear sometimes that the extended use and reliance on POCT and PONT among other things may undermine their clinical skills, increase unnecessary testing and lead to misleading results [83].

However, some diseases are restricted to small or poor areas in the world. Thus, in vitro diagnostic companies have no economic interest to develop or commercialize a diagnostic test for these neglected diseases [84].

\section{The need for capacity building at the link between human and animal health}

Seventy-five percent of human infectious diseases are zoonotic from animal origin [85]. Therefore, diagnostic tools for detecting these infectious agents from both human and animals are highly necessary. One of the causes leading to the emergence of pathogens in many African countries is bush meat, since a large proportion of the population depends on it as nutrition source. Not only the consumption but also the processing of the meat can lead to an infection [86]. Also, settlement in forests and deforestation are driving factors in the spread of infectious diseases as habitats of pathogens' vectors and reservoirs shrink and start to overlap with areas where domesticated animals and people live [87]. The danger of being infected by a zoonotic disease is not limited to low resource settings. In general, every person who lives or works in close contact to animals is at risk [88]. This is particularly true for people living on a farm or together with pets but also for people in contact with wild or zoo animals [89].

Unfortunately, there is a lack of diagnostic capacity in many regions of the world [9092]. For instance, in 2013, only twelve countries in sub-Saharan Africa have laboratories accredited to international standards. Ninety-one percent of the 380 laboratories were located in South-Africa [93]. These numbers show the urgent need of diagnostic capacity in sub-Saharan Africa. 


\section{The potential of Point of Care and point of need diagnostics in natural disasters}

When natural disasters happen, they are often followed by infectious disease outbreaks. Normally an outbreak starts during the post-impact phase within the first four weeks after the disaster. Depending on the type of the catastrophe, the resulting disease differs. For instance, floods are leading more often to mosquito borne infections, while earthquakes are correlated with diseases occurring due to contaminated food or water sources [94]. The WHO defines a relatively small number of diseases. Interviews with experts involved in previous disaster situations revealed that there are several important pathogens, which are listed in Table 5 [95].

Table 5: Important pathogens related with epidemic outbreaks after natural disasters as defined by Brock et al. [95] in comparison to pathogens listed by the WHO [96].

\begin{tabular}{|c|c|c|}
\hline & Brock et al., & $\begin{array}{c}\text { World Health } \\
\text { Organzation }\end{array}$ \\
\hline 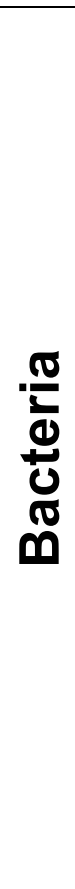 & $\begin{array}{l}\text { methicillin-resistant Staphylococcus aureus } \\
\text { E. coli } \\
\text { Pseudomonas aeruginosa } \\
\text { methicillin-sensetive Staphylococcus aureus } \\
\text { Enterobacter } \\
\text { Klebsiella } \\
\text { Enterococcus faecalis } \\
\text { Coagulase-negative Staphylococcus } \\
\text { Streptococcus pyogenes } \\
\text { Enterococcus faecium } \\
\text { Serratia marcescens } \\
\text { Streptococcus agalactiae } \\
\text { Streptococcus viridans } \\
\text { Acinetobacter baumanii } \\
\text { Stenotrophomonas maltophilia }\end{array}$ & $\begin{array}{l}\text { Vibrio cholerae } \\
\text { E. coli } \\
\text { Clostridium tetani }\end{array}$ \\
\hline \multirow[b]{2}{*}{ 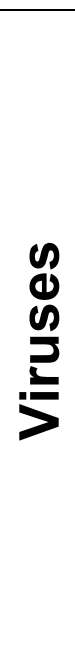 } & $\begin{array}{l}\text { Human Immunodeficiency virus } \\
\text { Hepatitis B virus } \\
\text { Hepatitis C virus } \\
\text { West Nile virus }\end{array}$ & $\begin{array}{l}\text { Hepatitis A } \\
\text { Hepatitis E } \\
\text { Measles virus } \\
\text { Dengue fever virus }\end{array}$ \\
\hline & $\begin{array}{l}\text { Human T-lymphotropic virus } \\
\text { Cytomegalovirus } \\
\text { West-Nile virus } \\
\text { Dengue fever virus } \\
\text { Epstein-Bar virus } \\
\text { Parvovirus B19 } \\
\text { Chikungunya virus }\end{array}$ & $\begin{array}{l}\quad \text { Other pathogens } \\
\text { Plasmodia species } \\
\text { Leptospira species } \\
\text { acute respiratory infections }\end{array}$ \\
\hline
\end{tabular}


Natural disasters like earthquakes, floods or hurricanes can lead to destruction or overloading of the local infrastructure. To face the threat of disease outbreak after a disaster, diagnostic tests must be handheld or portable, have fast turn-around times, good performance, be capable of multiple pathogen testing, and infectious material must be disposable easily [95].

POCT and PONT, like LFIA or portable nucleic acid amplification systems, have the ability to fulfil these requirements and can overcome the pitfalls of demolished diagnostic infrastructures. LFIA are small and can be sent fast to the disaster affected regions. The low-cost test can be used not only to test diseased individuals but also for prevention by testing water and food sources. Tests based on nucleic acid amplification can easily be adjusted to several pathogens. This eases the implementation of functional diagnostics in a region with destroyed infrastructure [97].

\section{Why the importance of point of care and point of need testing increases}

While globalisation and international travel progresses, the risk of emerging diseases is increasing [98]. Due to nowadays global flight connections, persons, goods, or traded animals can reach almost every location in the world within one or two days. The incubation times of several important zoonotic and animal diseases with emerging potential defined by OIE $[99,100]$ are longer than the flight time (Table 7$)$.

Table 6: Incubation times of selected important zoonotic and animal diseases.

\begin{tabular}{|l|c|}
\multicolumn{1}{|c|}{ Pathogen } & $\begin{array}{c}\text { Incubation time } \\
\text { (days) }\end{array}$ \\
\hline African Swine Fever virus & $5-21$ \\
\hline Suid herpesvirus 1(Aujeszky's disease) & $2-10$ \\
\hline Classical swine fever virus & $2-14$ \\
\hline Foot and Mouth Disease virus & $2-14$ \\
\hline Influenza viruses & $1-4$ \\
\hline Lumpy Skin Disease virus & $4-28$ \\
\hline Ebola virus & $2-21$ \\
\hline Marburg virus & $2-21$ \\
\hline Middle East respiratory syndrome virus & $2-14$ \\
\hline Rift valley fever virus & $2-6$ \\
\hline Severe acute respiratory syndrome virus & $2-7$ \\
\hline Hand, foot, and mouth disease viruses (Enterovirus) & $3-6$ \\
\hline
\end{tabular}


Around 75 percent of all new emerging diseases are zoonotic [85], and many diseases cause a similar clinical picture, for instance diarrhoea (20 different pathogens, [101]). Thus, tests detecting multiple pathogens across species borders are needed. Following the One Health concept, todays diagnostics need to cover a holistic approach and acknowledge the connections between human, animal, and environmental health [102].

\section{Aim of the PhD work}

This $\mathrm{PhD}$ work addresses the need of the fast identification of the responsible agents causing similar clinical symptoms in an outbreak at nucleic acid and antibody level. Firstly, a method for the rapid and field applicable nucleic acid extraction from complex samples was developed based on magnetic beads and reverse purification technology. The Mycobacterium avium subspecies paratuberculosis (MAP) was used as model pathogen (Chapter II). ZIKV was employed to establish a field applicable protocol for the identification of RNA viruses by diagnostic metagenomics. Further, this protocol was adjusted to the rapid serotyping of Foot and Mouth disease virus (FMDV) relying on nanopore sequencing and offline BLAST search (Chapter III and IV).

Finally, a peptide microarray was used to determine a bundle of ZIKV specific antibody target regions (ATRs) in order to unearth serological markers for the better differentiation of ZIKV in the background of other flaviviruses (Chapter $V$ ) with the aim of establishing a simple diagnostic method. 


\section{Chapter II:}

Development of Rapid Extraction Method of Mycobacterium avium subspecies paratuberculosis DNA from Bovine Stool Samples

Sören Hansen ${ }^{1}$, Marco Roller², Lamia Alslim ${ }^{1}$, Susanne Böhlken-Fascher ${ }^{1}$, Kim

Fechner $^{1}$, Claus-Peter Czerny ${ }^{1}$, Ahmed Abd El Wahed $^{1 *}$

1) Division of Microbiology and Animal Hygiene, Department of Animal Sciences, Faculty of Agricultural Sciences, University of Goettingen, Goettingen, Germany

2) Zoological and Botanical Garden Wilhelma, Stuttgart, Germany

${ }^{*}$ Corresponding Author 
Article

\title{
Development of Rapid Extraction Method of Mycobacterium avium Subspecies paratuberculosis DNA from Bovine Stool Samples
}

\author{
Sören Hansen ${ }^{1}$, Marco Roller ${ }^{2}$, Lamia M. A. Alslim ${ }^{1}$, Susanne Böhlken-Fascher ${ }^{1}$, Kim Fechner ${ }^{1}$, \\ Claus-Peter Czerny ${ }^{1}$ and Ahmed Abd El Wahed ${ }^{1, *}$ \\ 1 Division of Microbiology and Animal Hygiene, Department of Animal Sciences, Faculty of Agricultural \\ Sciences, University of Goettingen, D-37077 Goettingen, Germany; soeren.hansen@uni-goettingen.de (S.H.); \\ lamia.alslim@hotmail.com (L.M.A.A.); susanne.boehlken-fascher@agr.uni-goettingen.de (S.B.-F.); \\ kfechne@gwdg.de (K.F.); cczerny@gwdg.de (C.-P.C.) \\ 2 Zoological and Botanical Garden Wilhelma, D-70376 Stuttgart, Germany; marco_roller@web.de \\ * Correspondence: abdelwahed@gwdg.de
}

Received: 29 January 2019; Accepted: 27 March 2019; Published: 29 March 2019

\begin{abstract}
The rapid identification of Mycobacterium avium subspecies paratuberculosis (MAP) infected animals within the herd is essential for preventing the spread of the disease as well as avoiding human exposure. Although culture is seen as the gold standard, there are various molecular assays available i.e., polymerase chain reaction (PCR) or isothermal amplification technique (recombinase polymerase amplification (RPA)) for the detection of MAP. The accuracy of the molecular assays is highly dependent on the DNA extraction method. In order to establish a rapid point of need system for the detection of MAP DNA from stool samples, we developed a rapid DNA extraction protocol (MAP DNA SpeedXtract) specified for use in combination with the RPA. The whole procedure from "sample in" to "result out" was conducted in a mobile suitcase laboratory. The DNA extraction is based on reverse purification by magnetic beads, which reduces the required technical demand. The MAP DNA SpeedXtract was performed within $25 \mathrm{~min}$ and only three pipetting steps were needed. The amplification and detection time were $20 \mathrm{~min}$ in RPA. The sensitivity and specificity of the developed protocol in comparison with the lab-based silica membrane column extraction and real-time PCR were $90.9 \%(n=22)$ and 100\% $(n=23)$, respectively. In conclusion, we established a rapid and reliable protocol for the extraction and detection of MAP DNA. All reagents are cold chain independent. The entire setup is ideal for point of need identification of MAP infected cases.
\end{abstract}

Keywords: Mycobacterium avium subsp. paratuberculosis; rapid extraction; mobile suitcase laboratory; SpeedXtract; point of need extraction

\section{Introduction}

The Mycobacterium avium subspecies paratuberculosis (MAP) as the causing agent for Johne's Disease (Paratuberculosis) in ruminants is a Gram-positive, aerobic, non-motile, non-spore-forming and acid fast bacterium [1]. Clinical signs of John's disease, such as weight loss, reduction in milk production and progressing diarrhoea, have an enormous negative impact on the dairy industry [2]. Transmission occurs mainly through the faecal-oral route [3]. The identification of subclinical shedders is highly necessary to prevent silent spreading of the pathogen within the herd. Faecal culture is the gold standard for the diagnosis of MAP [4], however it requires at least 12-16 weeks before the sample can be considered as negative. Alternatively, highly sensitive and specific molecular assays such as Polymerase Chain Reaction (PCR) [5] or the recently published Recombinase Polymerase Amplification assay for the detection of MAP (MAP RPA) [6] are available. However, the clinical 
performance of these assays depends strongly on the quality of the extracted DNA [7]. Isolation of MAP DNA from faecal samples is especially challenging due to the presence of other complex compounds in the bovine faeces, which can inhibit the amplification process. Moreover, the cell walls of MAP, containing high numbers of lipophilic molecules and polysaccharides, are not easy to destroy [8-10]. In standard laboratory protocols, physical disruption is applied after adding chaotropic salts and proteinases to the sample. A lab tube containing silica gel membrane columns is used to obtain a highly purified DNA after employing several washing and centrifugation steps [7,11,12]. These procedures are often time consuming, complex and must be conducted at a well-equipped laboratory. In order to provide a diagnostic tool for paratuberculosis at point of need, here we described a rapid extraction protocol (MAP DNA SpeedXtract) based on magnetic bead. The destruction of the MAP cell wall in the SpeedXtract depends on the combination of physical disruption and heat in the presence of a lysis buffer. The magnetic beads capture the cell debris and most contaminants and then leave the nucleic acid free in the supernatant [13-15]. Therefore, the SpeedXtract was named a reverse purification method.

\section{Materials and Methods}

\subsection{Sample Origin}

The study included 45 bovine stool samples which were collected during routine veterinary examination in Division of Microbiology and Animal Hygiene, Goettingen. All samples were taken under consideration of the German codex "Gute Veterinärmedizinische Praxis".

\subsection{Development of MAP SpeedXtract Protocol}

In order to establish a rapid point of need nucleic acid extraction method, 11 different pre-treatment steps (Table 1) were combined with a basic SpeedXtract procedure. All methods were evaluated using bovine faecal sample containing intact MAP particles.

The basic SpeedXtract (QIAgen, Hilden, Germany) was performed as follows: $500 \mu \mathrm{L}$ of lysis buffer (Buffer SL) and $60 \mu \mathrm{L}$ of magnetic beads were added to the faecal samples. The mix was vortexed for $10 \mathrm{~s}$ and incubated at $95^{\circ} \mathrm{C}$. Every two minutes, the tube was taken out from the heat block and vortexed. Following $15 \mathrm{~min}$ of incubation time, the tube was placed on a magnetic rack. After two minutes, $10 \mu \mathrm{L}$ of the supernatant was diluted in $40 \mu \mathrm{L}$ nuclease free water. 


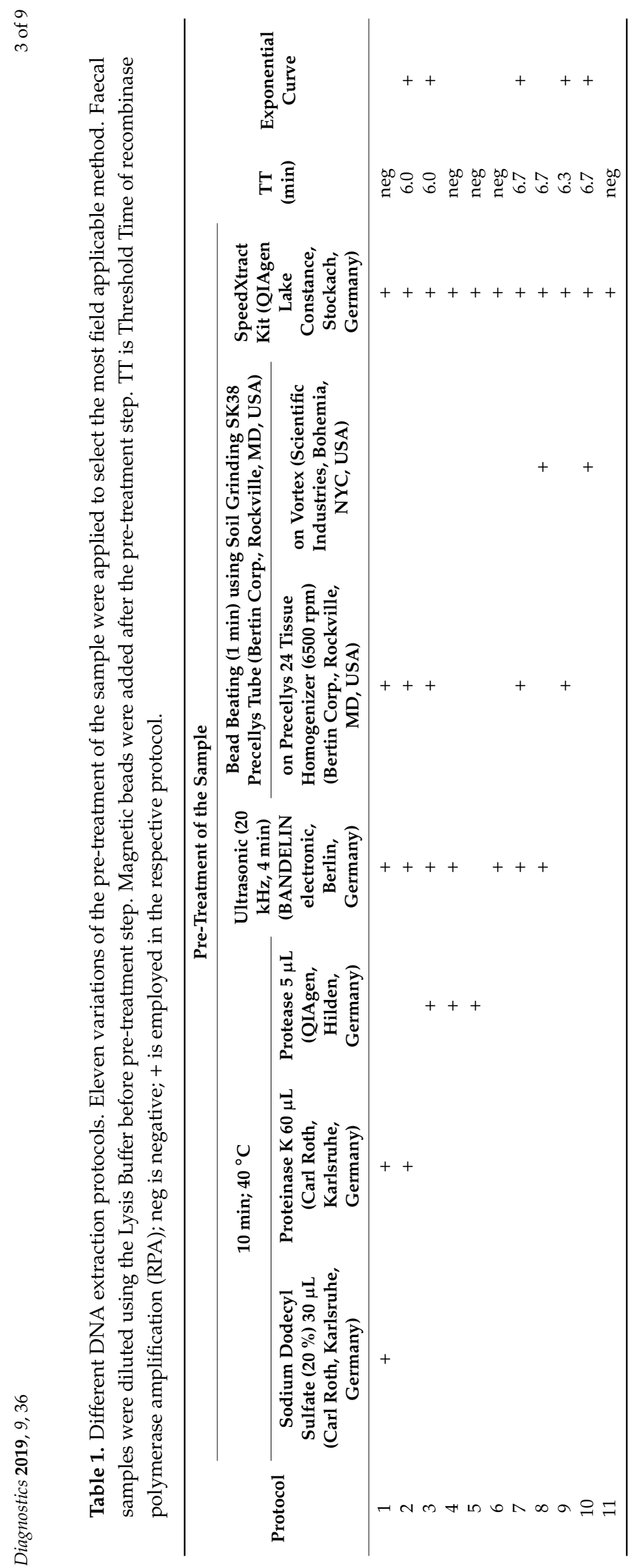




\subsection{RPA Assay}

The RPA assay was conducted as published previously [6]. Briefly, $5 \mu \mathrm{L}$ of the diluted supernatant, $29.5 \mu \mathrm{L}$ Rehydration Buffer, $6.7 \mu \mathrm{L}$ molecular biology water, $2.1 \mu \mathrm{L}$ of $10 \mu \mathrm{M}$ of both Forward Primer (5'-CGTGGACGCCGGTAAGGCCGACCATTACTGCATGG-3') and Reverse Primer (5'-CGCCGCAATCAACTCCAGCAGCGCGGCCTC-3'), $0.6 \mu \mathrm{L}$ of the $10 \mu \mathrm{M}$ of the exo probe (5'-ACGCCGGTAAGGCCGACCATTACTGCATGGT BHQ1-dt, Tetrahydrofuran and Fam-dT TAACGACGACGCGCA-PH-3') and $2.5 \mu \mathrm{L}$ of $14 \mathrm{mM}$ Mg acetate were added to a freeze-dried reaction pellet ordered from TwistDx (TwistDx Ltd., Cambridge, UK). The tube was incubated at $42{ }^{\circ} \mathrm{C}$ for 15 min. The fluorescence signals were recorded every $30 \mathrm{~s}$. The RPA threshold time was calculated using the first derivative value obtained by the Studio Software (Qiagen Lake Constance, Stockach, Germany).

\subsection{Clinical Sensitivity and Specificity}

The clinical performance of the selected MAP DNA SpeedXtract protocol in combination with the MAP RPA was validated using $100 \mathrm{mg}$ of each of the 45 clinical samples. From the same samples, DNA was extracted using the standard laboratory protocol (QIAamp DNA Blood Mini Kit, (QIAgen GmbH, Hilden, Germany)) and was screened with a well-established IS900 real-time PCR (FP: 5'-TACCGCGGCGAAGGCAAGAC-3'; RP: 5'-CGGAACGTCGGCTGGTCAGG-3' , probe: 5'-FAM-ATGACATCGCAGTCGAGCTG-BHQ-1-3'), as previously described [12].

\section{Results}

Eleven different pre-treatment steps in combination with a basic SpeedXtract procedure were tested to establish a rapid point of need nucleic acid extraction method. The performance of the extraction protocols was compared with the standard laboratory extraction method using a MAP-positive faecal sample. The results are summarized in Table 1 and Supplementary Figure S1. Protocol \#10 was selected as minimal pre-treatment steps and equipment were required (Figure 1), in addition to the production of a comparable result to the standard laboratory procedure (Figure 2). The whole procedure conducted in protocol \#10 is illustrated in Figure 1 and Supplementary File S1.
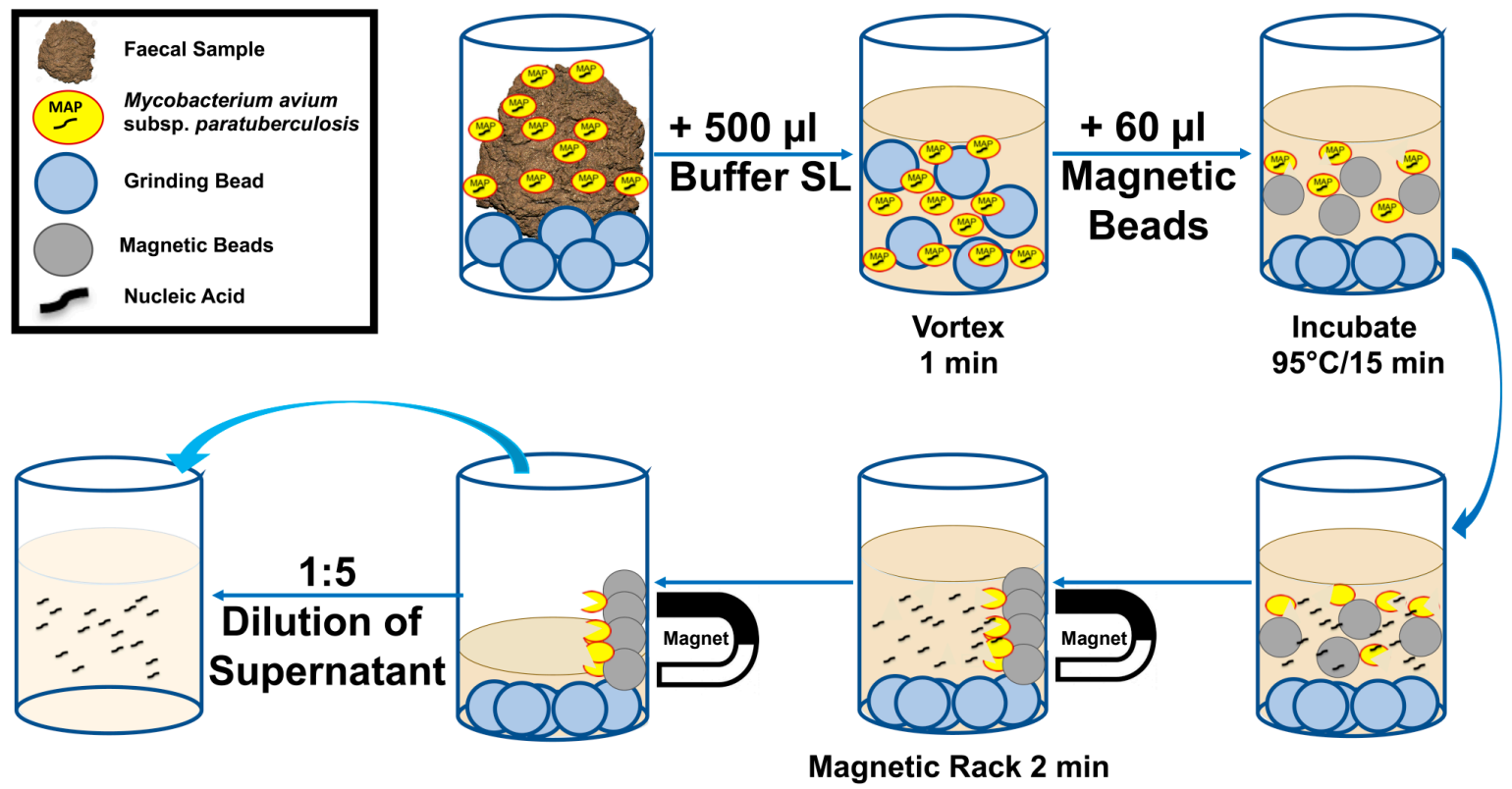

Figure 1. Workflow of the rapid point of need Mycobacterium avium subspecies paratuberculosis (MAP) extraction protocol. The procedure combines bead beating together with the basic SpeedXtract method. It represents protocol number 10 in Table 1. 


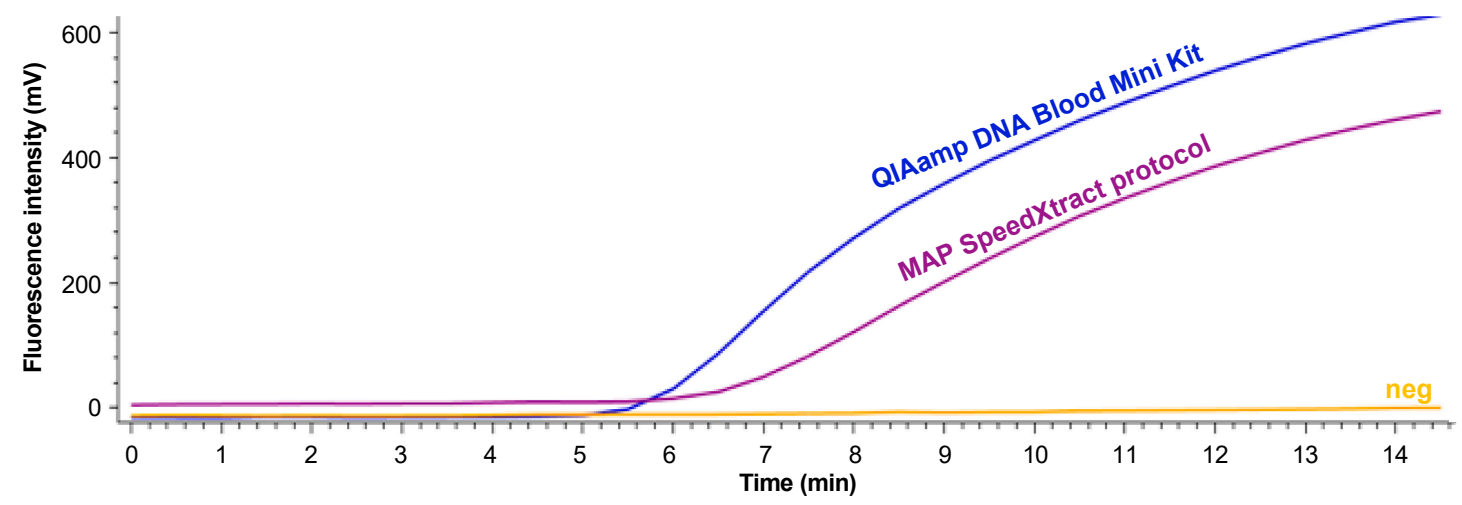

Figure 2. RPA results of DNA extracted either by using the QIAamp DNA Mini Blood Kit (blue) and the MAP SpeedXtract protocol (purple). Neg is negative.

Each of the 45 faecal samples were mixed well and divided into two parts (100 mg each). DNA was extracted from the first portion with the QIAamp DNA Blood Mini Kit and MAP DNA was detected with real-time PCR, while for the other portion, SpeedXtract and MAP RPA were applied. Comparing the results of both protocols revealed that 23/45 tested samples were negative by both methods. Twenty-two samples tested positive in the real-time PCR, while 20 were positive in the MAP RPA assay. No correlation between the threshold time of the RPA and cycle threshold of the real-time PCR was found (Figure 3).

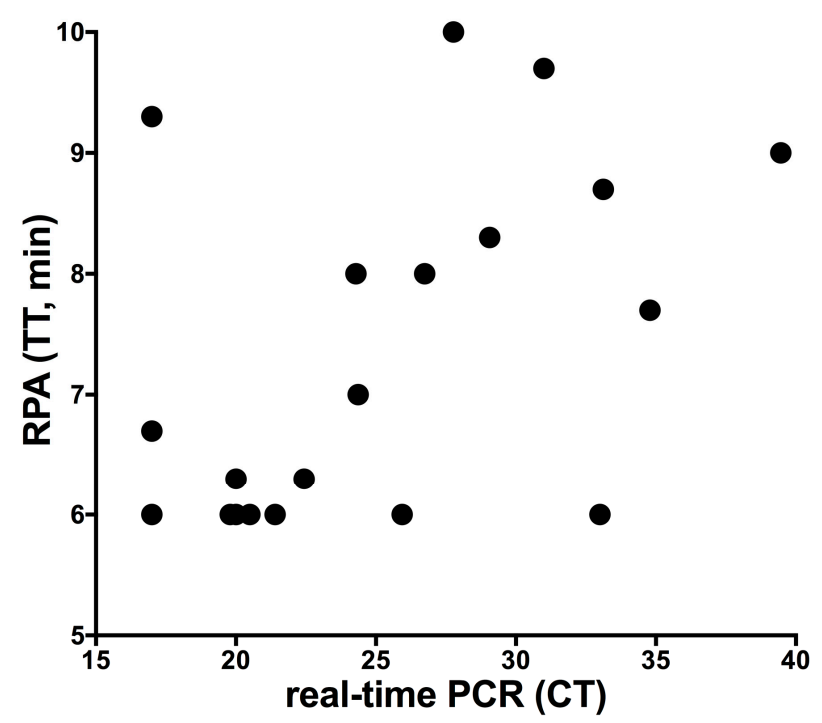

Figure 3. Results of clinical samples screened with both the MAP DNA SpeedXtract/MAP RPA protocol and the QIAamp DNA Blood Mini Kit/real-time Polymerase Chain Reaction (PCR) protocol. No correlation was found as the RPA assay was very fast, even with samples of high CT value. TT is threshold time; CT is cycle threshold.

\section{Discussion}

In this study, we developed a fast and easy to handle MAP DNA extraction and detection method, based on magnetic bead reverse purification and RPA, respectively. The complete procedure was optimized for use in the mobile suitcase laboratory [15]. The protocol reached the same clinical specificity and $90.9 \%$ sensitivity in comparison to the standard laboratory methods.

Many protocols for the extraction of MAP DNA have been developed in the past years (Table 2). All tested methods have showed outstanding clinical sensitivities, however long preparation time and several pipetting steps were necessary. This increases the risk of contamination, especially while 
working with bovine faecal samples at point of need. Leite et al. applied a rapid MAP extraction procedure [16], nevertheless, a high-speed centrifuge is needed and most centrifuges fail to work under field conditions [17]. Using the SpeedXtract removes the need of a high-speed centrifuge. In addition, the time from sample receiving to result including MAP RPA assay and handling is $45 \mathrm{~min}$ and only three pipetting steps are needed. The reverse purification technique, i.e., only inhibitors binding to the magnetic beads, can increase the yield of DNA since no multiple washing and elution steps are required. An additional benefit is that all reagents of the SpeedXtract Nucleic Acid Kit as well as the RPA are stable long term at room temperature, i.e., cold chain independent.

Mondal et al. and Gunaratna et al. applied the basic SpeedXtract Nucleic Acid Kit for the isolation of the Leishmania donovania DNA from a blood sample and skin biopsies, respectively $[13,15]$. Using the SpeedXtract virus kits, Weidmann et al. and Schlottau et al. isolated the Ebola and Rabies viral RNA from blood/swab samples and brain tissue, respectively $[18,19]$. Here is the first report on deploying the SpeedXtract for bacterial DNA isolation.

MAP colonies from middlebrook 7H11 agar plates have a high content of free DNA [20]. Therefore, spiking negative samples with a certain number of bacteria in order to determine the potency of the SpeedXtract was not useful. Thus, we relied on field samples to determine the clinical feasibility of the developed protocol.

The supernatant of the SpeedXtract did inhibit the real-time PCR as its colour stayed dark brown. In other words, the DNA extracted by SpeedXtract is not suitable for any applications including real-time PCR, however this is not the case with the RPA as the RPA is more resistant to an inhibitor than the real-time PCR [6].

The most difficult aspect in the DNA extraction is the lipophilic compounds of the MAP cell wall and clusters which resist acid or alkaline lysis buffers [21]. Bead beating is shown to increase the quality and quantity of yielded DNA [22]. The beads disrupt the cell wall and clusters by causing turbulences and mechanical shearing [23]. Therefore, a bead beating step was implemented in the protocol. As shown in Table 1, implementing treatment with ultrasonic or proteinase or protease gave no additional benefit to the performance in the MAP RPA (Supplementary Figure S1). 


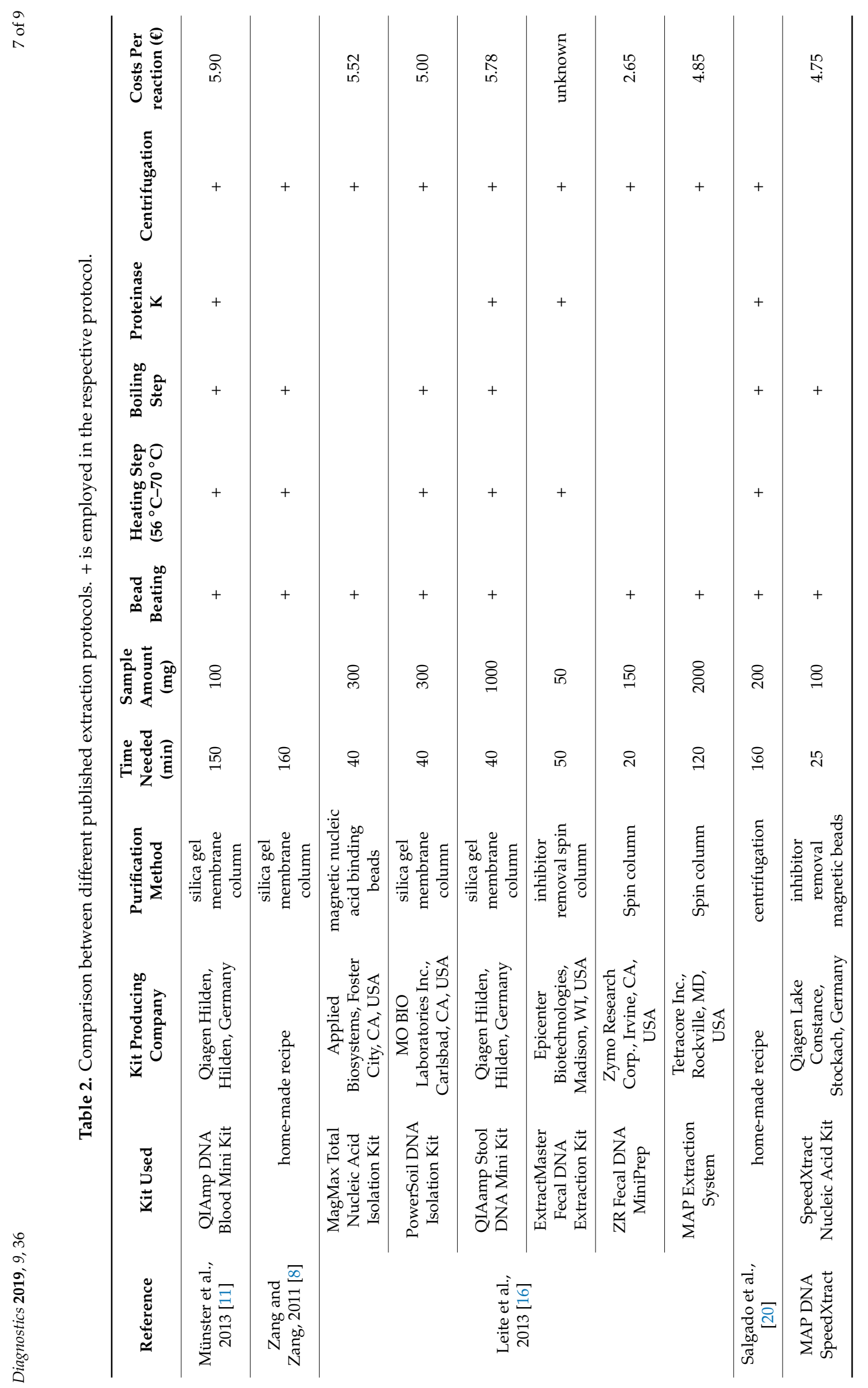




\section{Conclusions}

In conclusion, we developed a rapid and sensitive protocol for the extraction of MAP DNA. The uncomplicated setup as well as the minimal technical demand of the SpeedXtract and RPA methods enables implementation in a mobile suitcase laboratory [15]. This eases the detection of MAP shedders within the herd directly at the point of need.

Supplementary Materials: The following are available online at http://www.mdpi.com/2075-4418/9/2/36/s1, File S1: MAP SpeedXtract Protocol \#10; Figure S1: (A) RPA results of DNA extracted either by the QIAamp DNA Mini Blood Kit (blue) or the MAP SpeedXtract protocol with use of Proteinase K (purple) and without (pink). (B) Performance of the DNA extraction protocols \#7 to \#10.

Author Contributions: Conceptualization, S.H., A.A.E.W., and C.-P.C.; methodology, S.H., K.F., C.-P.C., and A.A.E.W.; software, S.H.; M.R., S.B.-F., and L.M.A.A.; validation, S.H., M.R., S.B.-F., and L.M.A.A.; formal analysis, S.H., M.R., S.B.-F., and L.M.A.A.; investigation, S.H., K.F., and A.A.E.W.; resources, A.A.E.W. and C.-P.C.; data curation, S.H., M.R., and A.A.E.W.; writing-original draft preparation, S.H. and A.A.E.W.; writing-review and editing, S.H., M.R., L.M.A.A., S.B.-F., K.F., C.-P.C., and A.A.E.W.; visualization, S.H., M.R., and A.A.E.W.; supervision, A.A.E.W. and C.-P.C.

Funding: This research received no external funding.

Acknowledgments: Authors thank Shereen Petersen Functional Imaging Unit, German Primate Center, Germany for English proofreading. We acknowledge the support of the Open Access Publication Funds of the University of Goettingen.

Conflicts of Interest: The authors declare no conflict of interest.

\section{References}

1. Manning, E.J.; Collins, M.T. Mycobacterium avium subsp. paratuberculosis: Pathogen, pathogenesis and diagnosis. Rev. Sci. Tech. 2001, 20, 133-150. [CrossRef] [PubMed]

2. Tiwari, A.; VanLeeuwen, J.A.; Dohoo, I.R.; Keefe, G.P.; Weersink, A. Estimate of the direct production losses in Canadian dairy herds with subclinical Mycobacterium avium subspecies paratuberculosis infection. Can. Vet. J. 2008, 49, 569-576. [PubMed]

3. Corbett, C.S.; De Buck, J.; Orsel, K.; Barkema, H.W. Fecal shedding and tissue infections demonstrate transmission of Mycobacterium avium subsp. paratuberculosis in group-housed dairy calves. Vet. Res. 2017, 48, 27. [CrossRef] [PubMed]

4. Albuquerque, P.P.; Santos, A.S.; Souza Neto, O.L.; Kim, P.C.; Cavalcanti, E.F.; Oliveira, J.M.; Mota, R.A.; Junior, J.W. Detection of Mycobacterium avium subsp. paratuberculosis in bovine milk from the state of Pernambuco, Brazil. Braz. J. Microbiol. 2017, 48, 113-117. [CrossRef]

5. Mobius, P.; Hotzel, H.; Rassbach, A.; Kohler, H. Comparison of 13 single-round and nested PCR assays targeting IS900, ISMav2, f57 and locus 255 for detection of Mycobacterium avium subsp. paratuberculosis. Vet. Microbiol. 2008, 126, 324-333. [CrossRef] [PubMed]

6. Hansen, S.; Schafer, J.; Fechner, K.; Czerny, C.P.; Abd El Wahed, A. Development of a Recombinase Polymerase Amplification Assay for Rapid Detection of the Mycobacterium avium subsp. paratuberculosis. PLoS ONE 2016, 11, e0168733. [CrossRef] [PubMed]

7. Mancini, N.; Carletti, S.; Ghidoli, N.; Cichero, P.; Burioni, R.; Clementi, M. The era of molecular and other non-culture-based methods in diagnosis of sepsis. Clin. Microbiol. Rev. 2010, 23, 235-251. [CrossRef] [PubMed]

8. Zhang, M.Z.; Zhang, S. An efficient DNA extraction method for polymerase chain reaction-based detection of Mycobacterium avium subspecies paratuberculosis in bovine fecal samples. J. Vet. Diagn. Invest. 2011, 23, 41-48. [CrossRef]

9. McNeil, M.R.; Brennan, P.J. Structure, function and biogenesis of the cell envelope of mycobacteria in relation to bacterial physiology, pathogenesis and drug resistance; some thoughts and possibilities arising from recent structural information. Res. Microbiol. 1991, 142, 451-463. [CrossRef]

10. Amaro, A.; Duarte, E.; Amado, A.; Ferronha, H.; Botelho, A. Comparison of three DNA extraction methods for Mycobacterium bovis, Mycobacterium tuberculosis and Mycobacterium avium subsp. avium. Lett. Appl. Microbiol. 2008, 47, 8-11. [CrossRef] [PubMed] 
11. Munster, P.; Volkel, I.; Wemheuer, W.; Schwarz, D.; Doring, S.; Czerny, C.P. A longitudinal study to characterize the distribution patterns of Mycobacterium avium ssp. paratuberculosis in semen, blood and faeces of a naturally infected bull by IS 900 semi-nested and quantitative real-time PCR. Transbound. Emerg. Dis. 2013, 60, 175-187. [CrossRef] [PubMed]

12. Fechner, K.; Schafer, J.; Wiegel, C.; Ludwig, J.; Munster, P.; Sharifi, A.R.; Wemheuer, W.; Czerny, C.P. Distribution of Mycobacterium avium subsp. paratuberculosis in a Subclinical Naturally Infected German Fleckvieh Bull. Transbound. Emerg. Dis. 2015, 64, 916-928. [CrossRef] [PubMed]

13. Gunaratna, G.; Manamperi, A.; Bohlken-Fascher, S.; Wickremasinge, R.; Gunawardena, K.; Yapa, B.; Pathirana, N.; Pathirana, H.; de Silva, N.; Sooriyaarachchi, M.; et al. Evaluation of rapid extraction and isothermal amplification techniques for the detection of Leishmania donovani DNA from skin lesions of suspected cases at the point of need in Sri Lanka. Parasit Vectors 2018, 11, 665. [CrossRef] [PubMed]

14. Faye, O.; Faye, O.; Soropogui, B.; Patel, P.; El Wahed, A.A.; Loucoubar, C.; Fall, G.; Kiory, D.; Magassouba, N.; Keita, S.; et al. Development and deployment of a rapid recombinase polymerase amplification Ebola virus detection assay in Guinea in 2015. Euro. Surveill 2015, 20. [CrossRef] [PubMed]

15. Mondal, D.; Ghosh, P.; Khan, M.A.; Hossain, F.; Bohlken-Fascher, S.; Matlashewski, G.; Kroeger, A.; Olliaro, P.; Abd El Wahed, A. Mobile suitcase laboratory for rapid detection of Leishmania donovani using recombinase polymerase amplification assay. Parasit Vectors 2016, 9, 281. [CrossRef] [PubMed]

16. Leite, F.L.; Stokes, K.D.; Robbe-Austerman, S.; Stabel, J.R. Comparison of fecal DNA extraction kits for the detection of Mycobacterium avium subsp. paratuberculosis by polymerase chain reaction. J. Vet. Diagn. Invest. 2013, 25, 27-34. [CrossRef] [PubMed]

17. Abd El Wahed, A.; Patel, P.; Faye, O.; Thaloengsok, S.; Heidenreich, D.; Matangkasombut, P.; Manopwisedjaroen, K.; Sakuntabhai, A.; Sall, A.A.; Hufert, F.T.; et al. Recombinase Polymerase Amplification Assay for Rapid Diagnostics of Dengue Infection. PLoS ONE 2015, 10, e129682. [CrossRef]

18. Weidmann, M.; Faye, O.; Faye, O.; Abd El Wahed, A.; Patel, P.; Batejat, C.; Manugerra, J.C.; Adjami, A.; Niedrig, M.; Hufert, F.T.; et al. Development of Mobile Laboratory for Viral Hemorrhagic Fever Detection in Africa. J. Infect. Dis. 2018, 218, 1622-1630. [CrossRef] [PubMed]

19. Schlottau, K.; Freuling, C.M.; Muller, T.; Beer, M.; Hoffmann, B. Development of molecular confirmation tools for swift and easy rabies diagnostics. Virol. J. 2017, 14, 184. [CrossRef]

20. Salgado, M.; Verdugo, C.; Heuer, C.; Castillo, P.; Zamorano, P. A novel low-cost method for Mycobacterium avium subsp. paratuberculosis DNA extraction from an automated broth culture system for real-time PCR analysis. J. Vet. Sci. 2014, 15, 233-239. [CrossRef] [PubMed]

21. Gomez, V.; Guzman, A.; Mejia, G.; Caceres, D.; Robledo, J.; Rouzaud, F. Evaluation of Simple and Cost-Effective DNA Preparation and Subsequent PCR Amplification for Clinically Relevant Mycobacteria. Br. J. Med. Med. Res. 2015, 8, 147-156. [CrossRef]

22. Tell, L.A.; Foley, J.; Needham, M.L.; Walker, R.L. Comparison of four rapid DNA extraction techniques for conventional polymerase chain reaction testing of three Mycobacterium spp. that affect birds. Avian. Dis. 2003, 47, 1486-1490. [CrossRef] [PubMed]

23. QIAgen; (Hilden; Germany). RNeasy®Mini Handbook. Available online: https://www.arabidopsis.org/ download_files/Protocols/RNeasy.pdf (accessed on 29 March 2019).

(C) 2019 by the authors. Licensee MDPI, Basel, Switzerland. This article is an open access article distributed under the terms and conditions of the Creative Commons Attribution (CC BY) license (http://creativecommons.org/licenses/by/4.0/). 


\section{Chapter III:}

\section{Combination random isothermal amplification and nanopore sequencing for rapid identification of the causative agent of an \\ outbreak}

Sören Hansen ${ }^{1}$, Oumar Faye ${ }^{2}$, Sabri S. Sanabani ${ }^{3}$, Martin Faye ${ }^{2}$, Susanne BöhlkenFascher $^{1}$, Ousmane Faye ${ }^{2}$, Amadou A. Sall ${ }^{2}$, Michaël Bekaert ${ }^{4}$, Manfred Weidmann ${ }^{4}$, Claus-Peter Czerny ${ }^{1}$, Ahmed Abd El Wahed ${ }^{1 *}$

\footnotetext{
1) Division of Microbiology and Animal Hygiene, University of Goettingen, Burckhardtweg 2, 37077 Goettingen, Germany

2) Institut Pasteur de Dakar, 43 Avenue Pasteur, BP 220, Dakar, Senegal

3) Department of Pathology, School of Medicine, R. Teodoro Sampaio, 115, University of São Paulo, São Paulo, Brazil

4)Institute of Aquaculture, University of Stirling, Stirling, FK9 4LA Stirling, Scotland, UK

*Corresponding Author
} 


\title{
Combination random isothermal amplification and nanopore sequencing for rapid identification of the causative agent of an outbreak
}

\author{
Ahmed Abd El Wahed ${ }^{\mathrm{a}, *}$ \\ a Division of Microbiology and Animal Hygiene, University of Goettingen, Burckhardtweg 2, 37077 Goettingen, Germany \\ ${ }^{\mathrm{b}}$ Institut Pasteur de Dakar, 43 Avenue Pasteur, BP 220, Dakar, Senegal \\ ${ }^{\mathrm{c}}$ Department of Pathology, School of Medicine, R. Teodoro Sampaio, 115, University of São Paulo, São Paulo, Brazil \\ ${ }^{\mathrm{d}}$ Institute of Aquaculture, University of Stirling, FK9 4LA Stirling, Scotland, UK
}

Sören Hansen ${ }^{\mathrm{a}}$, Oumar Faye ${ }^{\mathrm{b}}$, Sabri S. Sanabani ${ }^{\mathrm{c}}$, Martin Faye ${ }^{\mathrm{b}}$, Susanne Böhlken-Fascher ${ }^{\mathrm{a}}$, Ousmane Faye $^{\mathrm{b}}$, Amadou A. Sall ${ }^{\mathrm{b}}$, Michaël Bekaert ${ }^{\mathrm{d}}$, Manfred Weidmann ${ }^{\mathrm{d}}$, Claus-Peter Czerny ${ }^{\mathrm{a}}$,

\section{A R T I C L E I N F O}

\section{Keywords:}

Nanopore sequencing

Random isothermal amplification

Point of need diagnostics

Zika virus

\begin{abstract}
A B S T R A C T
Background: Outbreaks of fever of unknown origin start with nonspecific symptoms and case definition is only slowly developed and adapted, therefore, identifying the causative agent is crucial to ensure suitable treatment and control measures. As an alternative method for Polymerase Chain Reaction in molecular diagnostics diagnostic, metagenomics can be applied to identify the pathogen responsible for the outbreak through sequencing all nucleic acids present in a sample extract. Sequencing data obtained can identify new or variants of known agents.

Objectives: To develop a rapid and field applicable protocol to allow the identification of the causative agent of an outbreak.

Study design: We explored a sequencing protocol relying on multiple displacement isothermal amplification and nanopore sequencing in order to allow the identification of the causative agent in a sample. To develop the procedure, a mock sample consisting of supernatant from Zika virus tissue culture was used.

Results: The procedure took under seven hours including sample preparation and data analysis using an offline BLAST search. In total, 63,678 sequence files covering around 10,000 bases were extracted. BLAST search revealed the presence of Zika virus.

Conclusion: In conclusion, the protocol has potential for point of need sequencing to identify RNA viruses. The whole procedure was operated in a suitcase laboratory. However, the procedure is cooling chain dependent and the cost per sequencing run is still high.
\end{abstract}

\section{Background}

Identifying the causative agent implicated in an outbreak is crucial for selecting the suitable treatment and/or control measures [1]. For example, around 25 pathogens can cause influenza like symptoms in the acute phase [2] and up to 20 pathogens have to be considered for diarrhoea [3].

For direct detection of pathogens, polymerase chain reaction (PCR) is a widely used and well-established test for molecular diagnostics. Since specificity of PCR oligonucleotides depends on known sequences of specific target genes, false negative PCR result might be obtained due to a mismatching sequence of a novel variant of a known pathogen or because of a new emerging infectious agent. An alternative promising technology is diagnostic metagenomics, which can be applied to identify the pathogen responsible for the outbreak through sequencing of all nucleic acids in a sample allowing generic detection not limited by specific oligonucleotide design. Additionally, diagnostic metagenomics data sets on detected infectious agents can be used for phylogenetic and molecular epidemiological analysis to provide insights into strain and origin of the agent. This information can be crucial for organization and distribution of resources during the outbreak control $[4,5]$.

There are many high-throughput sequencing technologies available such as sequencing by synthesis, using HiSeq and MiSeq platform (Illumina, USA). These devices have a high data output, an error rate below $2 \%$ and the possibility to sequence several samples in parallel [6]. Nevertheless, there is a high logistic demand through weight, size

\footnotetext{
* Corresponding author.

E-mail addresses: abdelwahed@gwdg.de, abdelwahed@me.com (A. Abd El Wahed).
} 


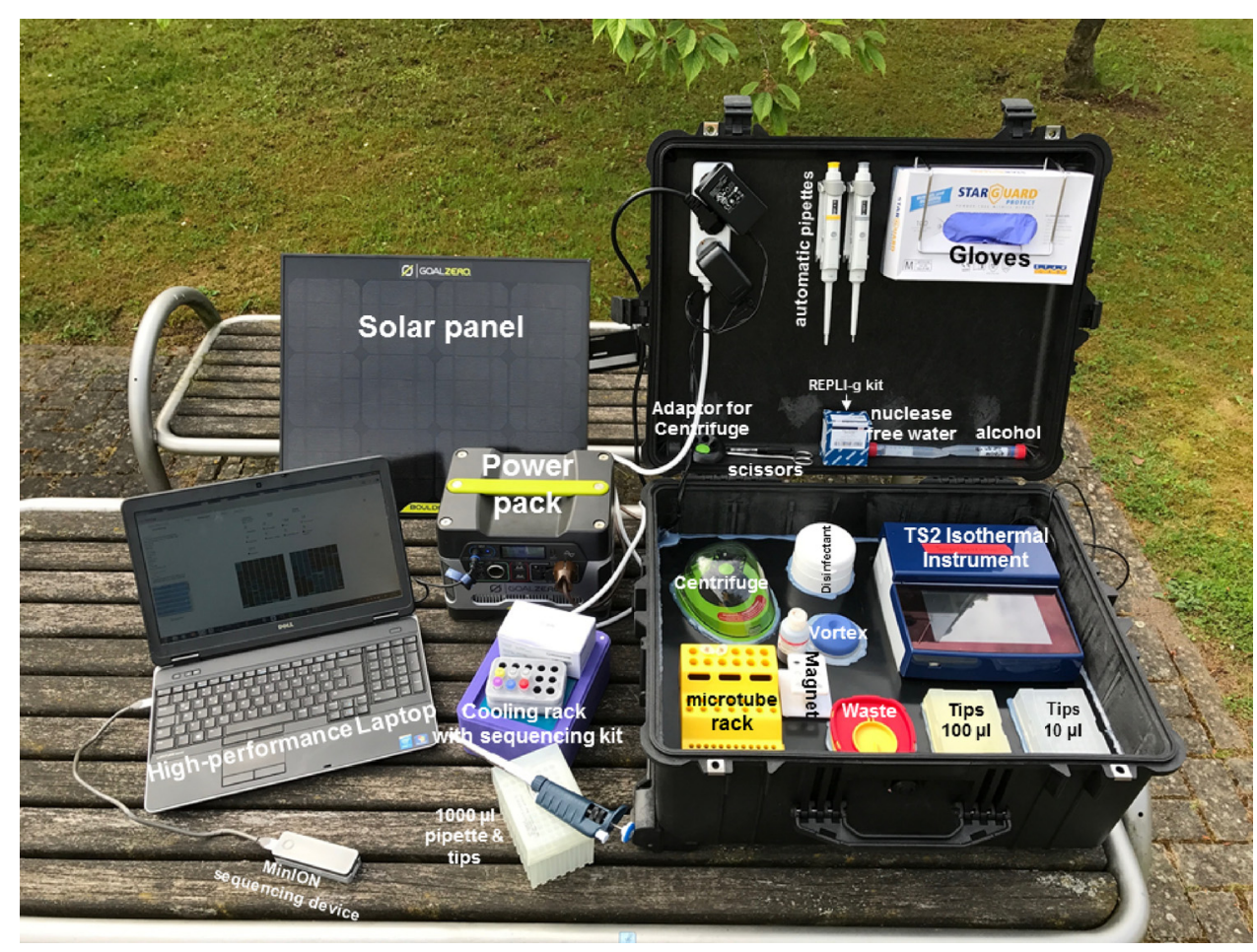

Fig. 1. The content of the mobile suitcase laboratory for sequencing.

and costs of the equipment as well as expensive service contracts. Furthermore, cumbersome and long sample and library preparation protocols are necessary in order to generate results [6,7]. In contrast, nanopore sequencing technology (Oxford Nanopore Technology, UK) uses a pore-protein embedded in a membrane to identify individual nucleotides by the unique change in electrical conductivity as a DNA molecule passes through the nanopore protein. Recently, Oxford Nanopore Technology developed a pocket sized $(10.5+3.5+2.5 \mathrm{~cm})$ sequencing device (MinION), which has the potential to be applied in the field or rural areas. A flow cell containing the required nanopores is inserted into the MinION in order to conduct the sequencing run. The MinION device operates at a constant sequencing temperature $\left(34^{\circ} \mathrm{C}\right)$ and translates the measured changes in current to a real-time nucleotide sequence via USB connection to a laptop [7-10].

\section{Objective}

Here we describe the establishment of a protocol for rapid identification of RNA viruses combining random isothermal amplification and nanopore sequencing using Zika virus (ZIKV) as model virus. The protocol was performed in a mobile suitcase laboratory (Fig. 1) in order to allow implementation in outbreak situation [11] at low resource settings.

\section{Study design}

\subsection{Sample origin}

The Zika virus strain HD78788 was isolated in 1991 in Senegal during surveillance. Viral stocks were prepared by inoculating viral strains into Aedes pseudoscutellaris clone 61 (AP61) monolayer cells. Viral infection was confirmed by an indirect immunofluorescence assay using specific hyper-immune mouse ascitic fluid as described previously [12].

\subsection{Sample preparation}

Zika virus (ZIKV) RNA was extracted from cell culture supernatant using the QIAamp Viral RNA Mini Kit (QIAGEN Hilden, Germany) following the manufacturer's instructions. The RNA quantity was measured by NanoDrop ND-1000 spectrometer (Thermo Scientific, Waltham, MA, USA). For elimination of genomic DNA and reverse transcription, the QuantiTect Reverse Transcription Kit (QIAGEN Hilden, Germany) was employed using a prolonged incubation time $(25 \mathrm{~min})$ for the reverse transcription step. Second strand cDNA Synthesis was performed with the NEBNext mRNA Second Strand Synthesis Module (New England Biolabs, Ipswich, MA, USA). The double-stranded cDNA (ds-cDNA) was purified with the 1.8X Agencourt AMPure XP Beads Kit (Beckman Coulter, Brea, CA, USA), eluted in $55 \mu \mathrm{L}$ nuclease-free water and quantified (NanoDrop ND-1000). To fragment and increase the amount of DNA, random amplification was done using the REPLI-g UltraFast Mini Kit (QIAGEN Hilden, Germany). Briefly, $1 \mu \mathrm{L}$ of ds-cDNA, containing at least $10 \mathrm{ng}$, was incubated with $1 \mu \mathrm{L}$ denaturation buffer at room temperature for three min. The reaction was terminated by addition of $2 \mu \mathrm{L}$ neutralization buffer. The denatured ds-cDNA was mixed with $16 \mu \mathrm{L}$ of the master mix containing $15 \mu \mathrm{L}$ REPLI-g UltraFalst reaction buffer and $1 \mu \mathrm{L}$ REPLI-g UltraFast DNA polymerase and incubated at $30{ }^{\circ} \mathrm{C}$ for $90 \mathrm{~min}$. The reaction mix was heated to $65^{\circ} \mathrm{C}$ for $3 \mathrm{~min}$ to inactivate the reaction enzymes. Then, the DNA was purified with the 1.8X Agencourt AMPure XP Beads Kit, eluted in $30 \mu \mathrm{L}$ nuclease free water and quantified (NanoDrop ND1000).

\subsection{Library preparation and sequencing}

For library preparation, the protocol for amplicon sequencing, SQKNSK007, was used as recommended by Oxford Nanopore Technology. Briefly, $45 \mu \mathrm{L}$ containing at least $1 \mu \mathrm{g}$ ds-cDNA were used for end-repairing and dA-tailing using the NEBNext Ultra II end-repair / dAtailing module. The end-repaired DNA was purified with the $1.8 \mathrm{X}$ Agencourt AMPure XP Beads Kit and eluted in $31 \mu \mathrm{L}$ nuclease free water. DNA recovery aim was at least $700 \mathrm{ng} / \mu \mathrm{L}$. Adapter ligation and 
tethering was carried out with the NEB Blunt/TA Ligase Master Mix. The DNA was purified using the Dynabeads ${ }^{\circledR}$ MyOne $^{\mathrm{TM}}$ Streptavidin C1 Kit (Thermo Fisher Scientific, Waltham, MA, USA) and dissolved in $25 \mu \mathrm{L}$ of elution buffer (Oxford Nanopore Technology, Oxford, UK). Six microliters of the adapted and tethered DNA was mixed with $31.5 \mu \mathrm{L}$ nuclease free water and $37.5 \mu \mathrm{L}$ of running buffer FM1 (Oxford Nanopore Technology, Oxford, UK) and then loaded into the R9 flow cell in the MinION device.

\subsection{Data processing}

The MinION device generates raw data in HDF5 format. The files were transformed to FASTQ format with poretools toolkit v0.6.0 [13]. Duplicate reads were deleted and the remaining sequences were used for a local BLAST search using Geneious v9.1.6 (Biomatters Ltd., Auckland, New Zealand). The custom BLAST database was built from all 7246 virus genome assemblies available on the NCBI databases (accessed the 2017-05-01). Contigs were aligned to ZIKV genome (accession KF383115.1) strain via "Map to Reference" option in GENEIOUS. A standard i5 Laptop using Windows 7 was used for all steps.

\section{Results}

The described procedure took less than seven hours ( $\sim 400 \mathrm{~min})$ as shown in Table 1 . In total, 63,678 sequences were extracted and transformed to FASTQ format. After running a local BLAST against a viral genome database (e-value threshold: $10^{-10}$ ), ZIKV sequences were identified in approximately $4 \%$ of the reads. The complete original ZIKV sequence (GenBank accession number: KF383115.1) was recovered with 2454 reads with an average read length of 685 . The maximum coverage rate was 585 reads and the minimum was 36 reads (Std. Dev.: 122.6). Pairwise identity in BLAST analysis was $67.4 \%$.

Additionally, a total of 411 correct ZIKV reads were found in the HDF5 failed call files. When included in the assembly they matched correctly to the respective ZIKV sequence (Fig. 2).

A total of 2043 pass and 411 fail reads were aligned to the ZIKV sequence (GenBank accession number: KF383115). The pass reads showed a better coverage rate (Max.: 190, Min.: 21, Std. Dev.: 40, Fig. 2 panel A), while the fail reads has a better sequence identity to the reference strain (Fig. 2 panel B).

\section{Discussion}

Identifying the causative agent of an outbreak using sequencing instead of other molecular techniques like PCR could have a high impact on selecting and implementing the right patient management and control measures.

The most widespread sequencing device is the MiSeq, as Illumina`s smallest device, which has nevertheless a size of $68.6+52.3+56.5 \mathrm{~cm}$ and a weight of approximately $57 \mathrm{~kg}$. Moreover, it has a higher data output (up to 15 Giga bases) in comparison to the MinION (10 Giga bases). Nevertheless, read length by MiSeq is limited to around $300 \mathrm{bp}$ and a maximum of $22-25$ million reads can be produced in a run time between $4 \mathrm{~h}$ and $56 \mathrm{~h}[14,15]$. In contrast, the MinION has through its nanopore technology no limit in read length and number. Moreover, reads are generated in $20-40 \mathrm{~min}$ and data are easily accessible on laptop.

We have found that $1 / 5$ of the correct ZIKV reads were classified as failed. MinKNOW platform categorises the reads into pass and fail reads by neuronal network computing assessing definite conductivity readout events at the pore exit for 5-6 mers. This complicated sequence definition needs quality scoring to decide on the statistical trustworthiness of the sequencing result. Fails are defined through the following approach. Initially base calling (1D base calling) of template and complement reads is performed separately. If the resulting sequence length ratio is between $0.5-2.0$, all sequences are stacked together for $2 \mathrm{D}$ base calling. If resultant $2 \mathrm{D}$ sequences are assessed with a mean Q-score $\leq 9$ they are sorted into a fail sequence file [16].

Short Illumina device reads have a $0.1 \%$ non-random error rate, which means an error at one site can still dominate the base calling process. The MinION reads have a $10 \%$ error rate but sites are distributed at random throughout the sequence which is compensated for by base calling and which therefore do not dominate at one site reducing the overall error rate in comparison to Illumina reads [17]. Our results suggest that the analysis algorithm and the Q-score need to be optimised for viral RNA sequences. At this current development stage, therefore, a recommended assembly approach would be first to use all pass reads to identify the infectious agent. To improve the result, the fail reads can be included in a $2^{\text {nd }}$ step.

The MinION was successfully used in the Ebola virus outbreak in Guinea [9] and during the Zika virus outbreak in Brazil [18]. In both cases, specific PCR fragment sequencing strategies were used. RT-PCR assays were applied to reverse transcribe RNA and create multiple fragments to increase the sequencing efficacy $[9,19]$. This strategy limits sequencing output to targeted agents, which is ideal for molecular epidemiological analysis. The use of PCR leads to logistic issues due to heavy devices and requirement of a cold chain for the reagents. In Brazil, this was solved by transporting the whole laboratory in a caravan. The generic sequencing approach described here is intended for diagnostic identification of unknown infectious agents. It uses only random isothermal steps throughout the procedure and PCR cycling is not required, which avoids the use of a thermal cycler.

We have already shown that isothermal amplification can be easily implemented in a mobile suitcase laboratory $[11,20,21]$ and we successfully adapted this concept for the workflow needed for library preparation for the MinION sequencing procedure (Fig. 1). The suitcase, contains all materials and reagents needed for sequencing in one box of $56.0+45.5+26.5 \mathrm{~cm}$ in size and less than $23 \mathrm{~kg}$ in weight.

All steps of data collection and analysis were performed offline using MinKNOW and Geneious. This is a major improvement since during the Ebola outbreak base calling for MinION datasets was only possible through cloud computing which needed internet capacity often not available locally [9]. The simple structure and clear layout of these analysis programmes makes it easy for users without bioinformatic background to obtain basic information about origin and phylogeny of the sequenced target. Therefore, a bioinformatician is not necessarily

Table 1

Sequencing Workflow.

\begin{tabular}{|c|c|c|}
\hline Diagnostic procedure & diagnostic tools (kit, device or software) & required time (min) \\
\hline RNA extraction & QIAamp Viral RNA Mini Kit & 30 \\
\hline DNA digestion and reverse transcription & QuantiTect Reverse Transcription Kit & 35 \\
\hline second strand cDNA synthesis & NEBNext mRNA Second Strand Synthesis Module & 90 \\
\hline random isothermal amplification & REPLI-g UltraFast Mini Kit & 120 \\
\hline library preparation & Nanopore sequencing kits: SQK-NSK007 & 70 \\
\hline Sequencing & MinION device, R9 flow cell and MinKNOW & 20 \\
\hline data analysis and BLAST search & PORETOOLS and Geneious 9.1.6 & 35 \\
\hline Total & & 400 \\
\hline
\end{tabular}




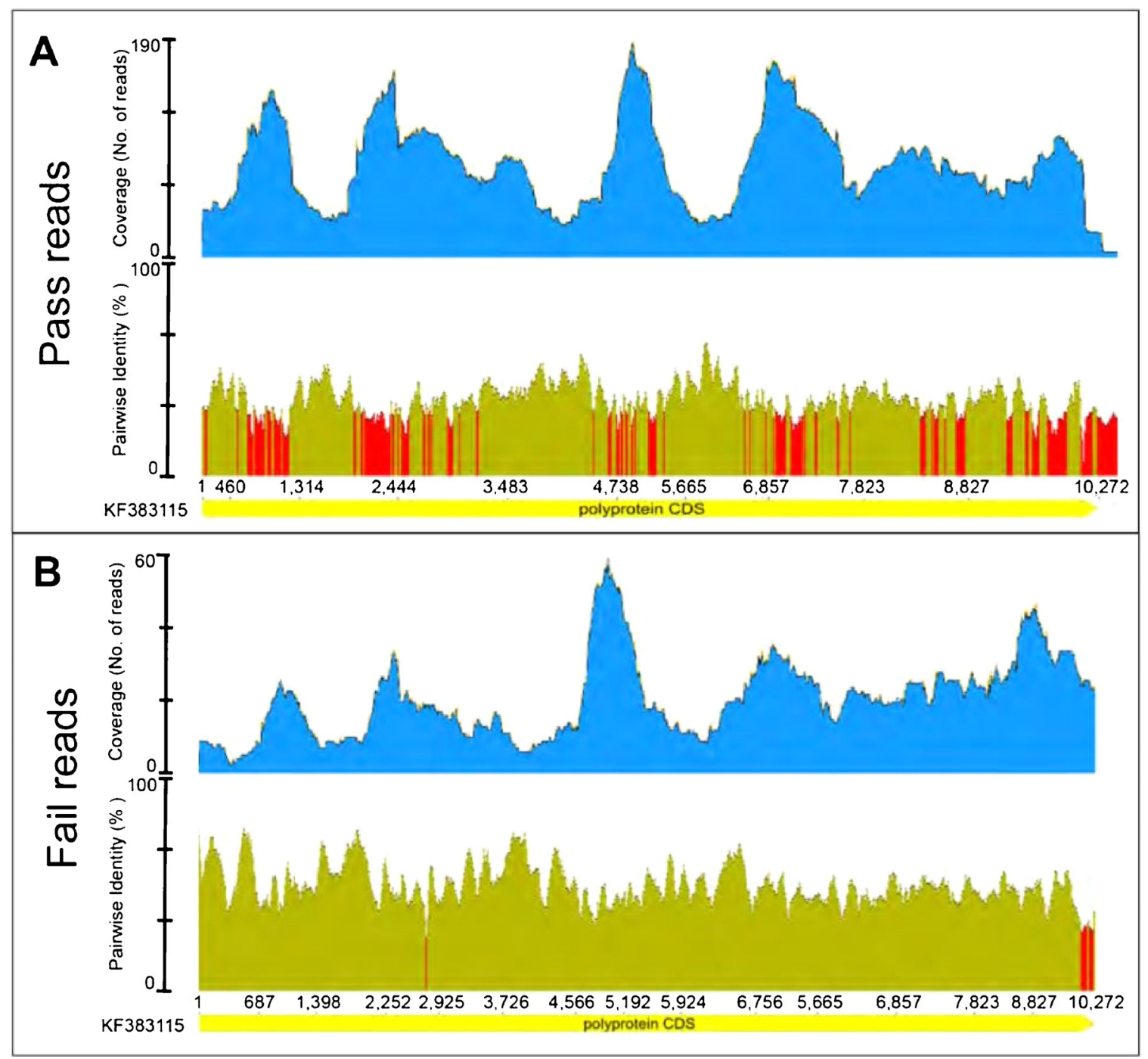

Fig. 2. Coverage rate (blue) and sequence identity (red and khaki) of pass (panel A) and fail (panel B) MinION sequence reads. Red is sequence identity below $30 \%$, while khaki is up to $100 \%$. The graph was created by GENEIOUS (For interpretation of the references to colour in this figure legend, the reader is referred to the web version of this article).

required for analysis of the datasets obtained in the field.

Currently, the following challenges have to be solved. In our hands, the sequencing reagents can be kept at $25^{\circ} \mathrm{C}$ for one day without any changes in their efficacy (confirmed by Oxford Nanopore Technologies, UK). However, for long-term storage a $-20^{\circ} \mathrm{C}$ freezer is still required. Moreover, the price per sequencing run is very high (around $\$ 1500$ ), as one flow cell costs between $\$ 500$ and $\$ 900$ depending on the amount of ordered flow cells. In addition, the shelf life of the flow cells is around 8 weeks at $4{ }^{\circ} \mathrm{C}$. One of the biggest drawbacks is that the manufacturer is progressively changing the reagents and flow cells so that it is difficult to match biochemistry to flow cells.

The goal of this study was to establish a protocol for pathogen identification during an outbreak field investigation. In principle, this seems possible in a suitcase laboratory setup. The next steps will be to identify cold chain independent reagents.

\section{Funding}

The project was partially funded by the Start-up Funding Program of the University of Goettingen, Germany (ID: 3,917,557). The funder has no rule in study or the decision to submit the manuscript.

\section{Data availability statement}

All data produced during this study was included in the manuscript. Original sequencing file are available upon request to the corresponding author.

\section{Competing interests}

None declared.

\section{Ethical approval}

Not required since no human or animal samples were used.

\section{Acknowledgment}

The project was partially funded by the Start-up Funding Program of the University of Goettingen, Germany (ID: 3917557).

\section{References}

[1] P.X. Ni, X. Ding, Y.X. Zhang, X. Yao, R.X. Sun, P. Wang, et al., Rapid detection and identification of infectious pathogens based on high-throughput sequencing, Chin. 
Med. J. (Engl.) 128 (2015) 877-883.

[2] H. Kelly, C. Birch, The causes and diagnosis of influenza-like illness, Aust. Fam. Phys. 33 (2004) 305-309.

[3] C.F. Lanata, C.L. Fischer-Walker, A.C. Olascoaga, C.X. Torres, M.J. Aryee, R.E. Black, et al., Global causes of diarrheal disease mortality in children $<5$ years of age: a systematic review, PLoS One 8 (2013) e72788.

[4] E.R. Robinson, T.M. Walker, M.J. Pallen, Genomics and outbreak investigation: from sequence to consequence, Genome Med. 5 (2013) 36.

[5] N.J. Croucher, X. Didelot, The application of genomics to tracing bacterial pathogen transmission, Curr. Opin. Microbiol. 23 (2015) 62-67.

[6] L. Liu, Y. Li, S. Li, N. Hu, Y. He, R. Pong, et al., Comparison of next-generation sequencing systems, J. Biomed. Biotechnol. 2012 (2012) 251364.

[7] T. Hoenen, A. Groseth, K. Rosenke, R.J. Fischer, A. Hoenen, S.D. Judson, et al, Nanopore sequencing as a rapidly deployable Ebola outbreak tool, Emerg. Infect. Dis. 22 (2016) 331-334.

[8] G.M. Cherf, K.R. Lieberman, H. Rashid, C.E. Lam, K. Karplus, M. Akeson, Automated forward and reverse ratcheting of DNA in a nanopore at 5-A precision, Nat. Biotechnol. 30 (2012) 344-348.

[9] J. Quick, N.J. Loman, S. Duraffour, J.T. Simpson, E. Severi, L. Cowley, et al., Realtime, portable genome sequencing for Ebola surveillance, Nature 530 (2016) 228-232.

[10] T. Laver, J. Harrison, P.A. O'Neill, K. Moore, A. Farbos, K. Paszkiewicz, et al., Assessing the performance of the Oxford nanopore technologies MinION, Biomol. Detect. Quantif. 3 (2015) 1-8.

[11] A. Abd El Wahed, M. Weidmann, F.T. Hufert, Diagnostics-in-a-suitcase: development of a portable and rapid assay for the detection of the emerging avian influenza
A (H7N9) virus, J. Clin. Virol. 69 (2015) 16-21.

[12] J.P. Digoutte, M.A. Calvo-Wilson, M. Mondo, M. Traore-Lamizana, F. Adam, Continuous cell lines and immune ascitic fluid pools in arbovirus detection, Res. Virol. 143 (1992) 417-422.

[13] N.J. Loman, A.R. Quinlan, Poretools: a toolkit for analyzing nanopore sequence data, Bioinformatics 30 (2014) 3399-3401.

[14] J.M. Urban, Bliss Jacob, Charles E. Lawrence, Susan A. Gerbi, Sequencing UltraLong DNA Molecules with the Oxford Nanopore MinION. BioRxiv, (2015).

[15] S. Junemann, F.J. Sedlazeck, K. Prior, A. Albersmeier, U. John, J. Kalinowski, et al., Updating benchtop sequencing performance comparison, Nat. Biotechnol. 31 (2013) 294-296.

[16] H. Lu, F. Giordano, Z. Ning, Oxford nanopore MinION sequencing and genome assembly, Genom. Proteom. Bioinf. 14 (2016) 265-279.

[17] S. Goodwin, J.D. McPherson, W.R. McCombie, Coming of age: ten years of nextgeneration sequencing technologies, Nat. Rev. Genet. 17 (2016) 333-351.

[18] J. Quick, N.D. Grubaugh, S.T. Pullan, I.M. Claro, A.D. Smith, K. Gangavarapu, et al., Multiplex PCR method for MinION and Illumina sequencing of Zika and other virus genomes directly from clinical samples, Nat. Protoc. 12 (2017) 1261-1276.

[19] N.R. Faria, E.C. Sabino, M.R. Nunes, L.C. Alcantara, N.J. Loman, O.G. Pybus, Mobile real-time surveillance of Zika virus in Brazil, Genome Med. 8 (2016) 97.

[20] O. Faye, O. Faye, B. Soropogui, P. Patel, A.A. El Wahed, C. Loucoubar, et al., Development and deployment of a rapid recombinase polymerase amplification Ebola virus detection assay in Guinea in 2015, Euro Surveill. 20 (2015).

[21] D. Mondal, P. Ghosh, M.A. Khan, F. Hossain, S. Bohlken-Fascher, G. Matlashewski, et al., Mobile suitcase laboratory for rapid detection of Leishmania donovani using recombinase polymerase amplification assay, Parasit. Vectors 9 (2016) 281. 


\section{Chapter IV:}

\section{Serotyping of foot-and-mouth disease virus using oxford nanopore sequencing}

Sören Hansen ${ }^{1 *}$, Veronika Dill ${ }^{2}$, Mohamed A. Shalaby ${ }^{3}$, Michael Eschbaumer ${ }^{2}$, Susanne Böhlken-Fascher ${ }^{1}$, Bernd Hoffmann ${ }^{2}$, Claus-Peter Czerny ${ }^{1}$, Ahmed Abd El Wahed $^{1}$

1) Division of Microbiology and Animal Hygiene, University of Goettingen, Burckhardtweg 2, D-37077 Goettingen

2) Institute of Diagnostic Virology, Friedrich-Loeffler-Institut, Federal Research Institute for Animal Health, Suedufer 10 , D-17493 Greifswald-Insel Riems, Germany.

3) Department of Virology, Faculty of Veterinary Medicine, Cairo University, 12211, Giza, Egypt

*Corresponding Author 
Protocols

\title{
Serotyping of foot-and-mouth disease virus using oxford nanopore sequencing
}

\author{
Sören Hansen ${ }^{\mathrm{a}, *}$, Veronika Dill ${ }^{\mathrm{b}}$, Mohamed A. Shalabyc, Michael Eschbaumer ${ }^{\mathrm{b}}$, \\ Susanne Böhlken-Fascher ${ }^{\mathrm{a}}$, Bernd Hoffmann ${ }^{\mathrm{b}}$, Claus-Peter Czerny ${ }^{\mathrm{a}}$, Ahmed Abd El Wahed ${ }^{\mathrm{a}}$ \\ a Division of Microbiology and Animal Hygiene, University of Goettingen, Burckhardtweg 2, D-37077 Goettingen, Germany \\ ${ }^{\mathrm{b}}$ Institute of Diagnostic Virology, Friedrich-Loeffler-Institut, Federal Research Institute for Animal Health, Suedufer 10, D-17493 Greifswald-Insel Riems, Germany \\ ${ }^{\mathrm{c}}$ Department of Virology, Faculty of Veterinary Medicine, Cairo University, 12211, Giza, Egypt
}

\section{A R T I C L E IN F O}

\section{Keywords:}

Foot and mouth disease virus

MinION

Nanopore sequencing

Oxford nanopore

Serotyping

\begin{abstract}
A B S T R A C T
Foot-and-mouth disease virus (FMDV), belonging to the family of Picornaviridae, infects mostly cloven-hoofed animals and leads to huge economic losses. Since there is no cross-protection between the seven serotypes of FMDV, effective vaccination relies on the knowledge of the serotype causing the outbreak. The most common methods of serotyping are antigen ELISAs and amplification-based sequencing. Serotype-specific PCR methods exist but have limitations due to emerging mutants within serotypes. Sequencing is a promising technology, but currently suffers from cumbersome procedures and long turnaround times. In this study, we have established a novel sequencing protocol relying on nanopore sequencing and offline BLAST search. The procedure was completed in $5 \mathrm{~h}$ including RNA extraction, reverse transcription, second-strand synthesis, barcoding, sequencing and data analysis, which did not require a bioinformatician. In total, 12,193 sequence files were obtained. The offline BLAST search to the P1 region revealed the most successful categorization of the seven FMDV serotypes (specificity: $98.3 \%$ ) over whole genome (24.8\%), P2 (23.6\%) and P3 (21.4\%). In conclusion, our protocol enables rapid and reliable FMDV serotyping. The whole procedure can be conducted with a mobile suitcase laboratory, which is easy to use at the point of need in endemic countries.
\end{abstract}

\section{Introduction}

Foot-and-mouth disease virus (FMDV) is a highly infectious positive single-stranded RNA virus belonging to the family of Picornaviridae. The virus affects mainly cloven-hoofed animals causing huge economic losses including high mortality in young stock, reduced herd fertility and productivity, trade restrictions as well as high cost for control programs. FMDV has seven serotypes (SAT1-3, Asia-1, A, O and C). Vaccines are available, but there is no cross-protection between the serotypes and tremendous variability within them. Thus, identifying the serotype and strain is crucial to select an appropriate vaccine to confine outbreaks (Knight-Jones and Rushton, 2013; Tekleghiorghis et al., 2014). Antigen ELISA with serotype-specific polyclonal sera is the most common method of serotyping FMDV (Roeder and Le Blanc Smith, 1987). However, the ELISA has limited sensitivity and is prone to crossreactions between serotypes. Type-specific RT-PCRs targeting the VP1encoding genome region are also applied for serotyping, both as conventional and real-time PCR is highly sensitive and specific, but most typing assays are tailored to a pool of viruses circulating in a defined region and may not be able to identify emerging strains or new viruses of unknown origin that are introduced into free areas (BachanekBankowska et al., 2016). Another promising way of serotyping is sequencing of the FMDV genome, which also gives more insight into the virus evolution and allows tracing the origin and spread of an outbreak. However, many current VP1 sequencing protocols are lengthy and cumbersome procedures including two amplification steps, in addition to the need of expensive equipment and reagents (Dill et al., 2017; Knowles et al., 2016; Longjam et al., 2011; Soltan et al., 2017).

In this study, we established for the first time a rapid sequencing protocol for FMDV serotyping using a nanopore sequencing method operated in a mobile suitcase laboratory.

\section{Material and methods}

\subsection{Viruses}

The FMDV isolates $A_{22}$ IRQ 24/64, $\mathrm{O}_{1}$ Manisa TUR/69, $\mathrm{C}_{1}$ Noville SWI/65, Asia-1 Shamir ISR/89, SAT1 ZIM 25/89, SAT2 ZIM 11/91 and

\footnotetext{
* Corresponding author.

E-mail address: soeren.hansen@uni-goettingen.de (S. Hansen).
} 
SAT3 ZIM 4/81 were selected from archival stocks at the FriedrichLoeffler-Institut (FLI), Germany.

\subsection{Sample preparation and RNA extraction}

Fresh cultures of all isolates were grown separately in monolayers of BHK-21 cells (RIE 164, Collection of Cell Lines in Veterinary Medicine, FLI). Infected cells were incubated in a closed system at $37^{\circ} \mathrm{C}$ until full cytopathic effect was visible. After freezing and thawing, the supernatant of the seven samples was clarified of cell debris by centrifugation for $10 \mathrm{~min}$ at $3200 \times g$ at $4{ }^{\circ} \mathrm{C}$. Virus was pelleted through a $30 \%$ (wt/ vol) sucrose cushion in $40 \mathrm{mM}$ sodium phosphate buffer ( $\mathrm{pH} 7.6)$ with $100 \mathrm{mM}$ sodium chloride (buffer P as in (Gullberg et al., 2013)) by centrifugation at $125,755 \times \mathrm{g}$ in a SW32Ti rotor (Beckman Coulter, Optima $170 \mathrm{LE}-70$ ) for $2 \mathrm{~h} 50 \mathrm{~min}$ at $7^{\circ} \mathrm{C}$. Pellets were resuspended in $140 \mu \mathrm{L}$ buffer P. FMDV RNA of the individual serotypes was extracted using the RNeasy ${ }^{\circledast}$ Mini Kit (Qiagen, Hilden, Germany) according to the manufacturer's instructions. RNA content was determined with a spectrophotometer (NanoDrop 2000c, Thermo Fisher Scientific) by repeated measurements of absorption at $260 \mathrm{~nm}$.

Under field conditions, where no centrifuge is available, the RNA extraction can be performed directly from vesicular material with the Dynabeads SILANE Viral Nucleic Acid kit (Invitrogen, Darmstadt, Germany) as described previously (Abd El Wahed et al., 2013).

\subsection{Library preparation}

The library preparation was carried out in the mobile suitcase laboratory using a direct cDNA native barcoding protocol (Fig. 1; the full procedure is shown in additional file 1). Firstly, reverse transcription and strand switching was performed with 250 ng of RNA using the In vitro SuperScript IV VILO Master Mix (ThermoFisher Scientific, Waltham, MA, USA) with the VNP primer targeting the poly-A tail of FMDV RNA (5'-phosphate-ACTTG CCTGT CGCTC TATCT TCTTT TTTTT TTTTT TTTTT TTVN-3') and strand-switching primer (5'-TTTCT GTTGG TGCTG ATATT GCTGC CATTA CGGCC-mGmGmG with 2' Omethyl RNA bases-3'), respectively. These primers were suggested by Oxford Nanopore Technologies; for details about their function, please refer to (Zhu et al., 2001). Secondly, 2nd strand synthesis was conducted using the LongAmp Taq Master Mix (New England Biolabs, Ipswich, MA, USA) and PR2 primer (5'-TTTCT GTTGG TGCTG ATATT GC- $3^{\prime}$ ). Thirdly, end-repairing was performed using the NEBNext Ultra II end-repair/dA-tailing module (New England Biolabs). Barcoding was carried out with the NEB Blunt/TA Ligase Master Mix (New England Biolabs) and Native Barcoding Kit 1D (NBD103, Oxford Nanopore Technologies, Oxford, UK) as follows: Barcodes 1, SAT1; 2, SAT2; 3, SAT3; 4, Asia-1; 5, A; 6, O; 7, C. After barcoding, the samples were pooled together. Fourthly, ligation of sequencing adaptor and tether attachment was done using the SQK-DCS108 sequencing kit (Oxford Nanopore) and NEB Blunt/TA Ligase Master Mix. Finally, the preparation was mixed with running buffer FM1 and Library Loading Beads Buffer (Oxford Nanopore) and loaded into an R9.4 flow cell fixed on the top of the MinION device (Oxford Nanopore).

\subsection{Data processing}

The sequencing run was performed in a MinION (Oxford Nanopore) within $20 \mathrm{~min}$. The data was automatically saved in FAST5 format on

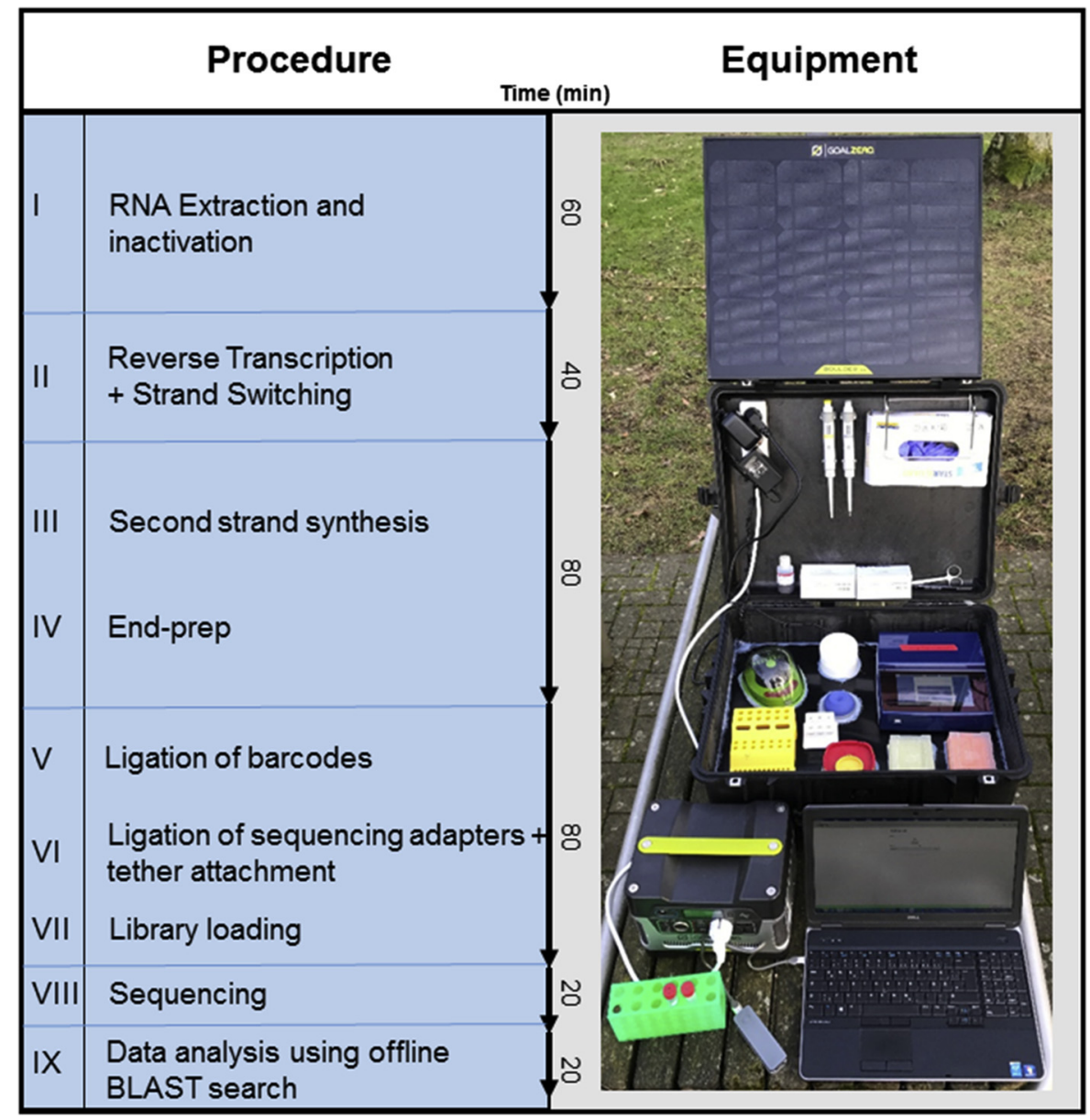

Fig. 1. Work flow and the required equipment for the serotyping of FMDV. 
the laptop via the MinKnow Software (Oxford Nanopore). The sequence files were automatically transferred to FASTQ format and categorized into barcodes using ALBACORE 2.0.1 (Oxford Nanopore). Duplicated reads and sequences less than 300 and more than 9000 nucleotides were deleted using GENEIOUS v9.1.6 (Biomatters Ltd., Auckland, New Zealand). Thereafter, sequences were aligned to offline local BLAST databases encompassing either P1, P2, P3 or the whole FMDV genome (for full sequence and accession numbers, please see additional file 2). The offline database was created ahead of time according to the procedure in the GENEIOUS manual. Local BLAST search was also performed using GENEIOUS applying the MEGABLAST algorithm and showing results as Query-centric alignment only. A maximum of 1000 hits per read was allowed with an E-value of 1e-100. Decision on the serotype was made based on the total number of possible GenBank data hits aligned to the nanopore sequence data. In order to determine the specificity of using the offline database of either the whole FMDV genome or particular gene region for serotyping, the number of hits from a particular serotype aligned correctly to sequence reads was divided by the total number of hits of all serotypes placed under the same barcode. A hit is one FMDV sequence with a distinct GenBank accession number.

\section{Results}

In total, 12,193 sequence files were generated and processed to FASTQ, from which 7372 could pass our sequence filtration. Categorization of the reads into the various barcodes is shown in Fig. 2. BLAST search revealed between 14.3 and 32.5 percent of the reads as FMDV sequences. Serotyping using BLAST searches to the whole genome as well as to sequences of the P2 and P3 region of FMDV revealed a very poor specificity, $24.8,23.6$ and $21.4 \%$, respectively. In contrast, applying the P1 database for alignment to the reads via MEGABLAST yielded the highest specificity (98.3\%).
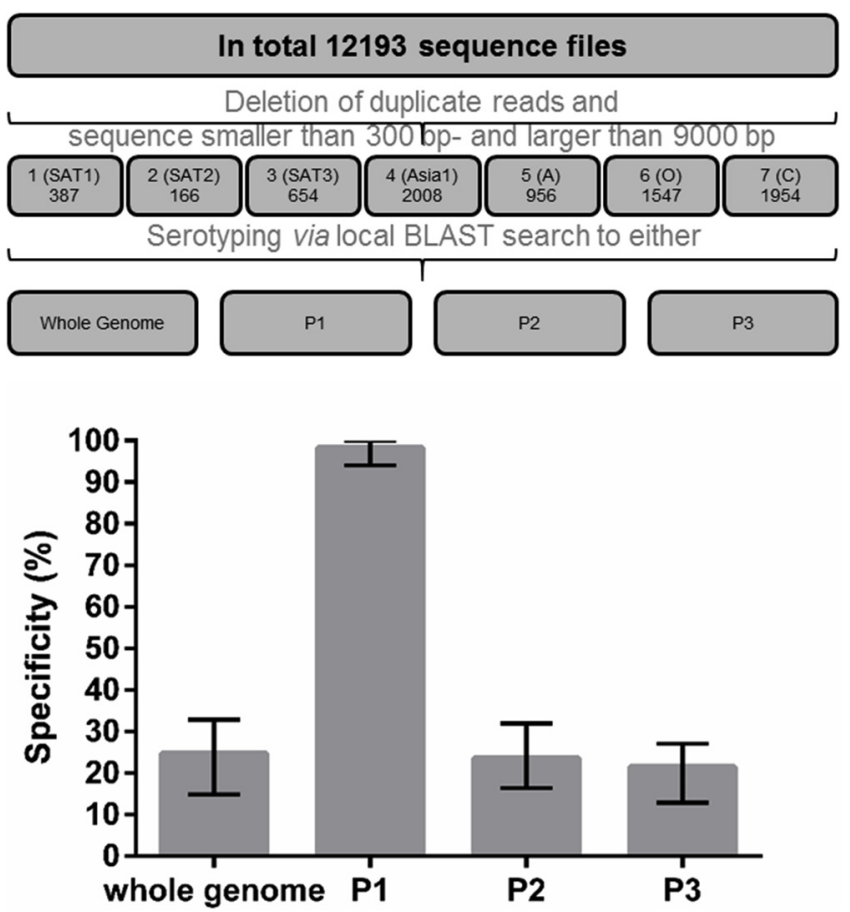

Fig. 2. Flow chart of analysing the sequence data using offline BLAST search. The lower graph shows the success rate of BLASTing the reads to the right FMDV serotype.

\section{Discussion}

We have developed a rapid sequencing method for serotyping of FMDV. Direct cDNA sequencing using a MinION device was combined with an offline local BLAST search in order to allow point-of-need FMDV serotyping. The whole protocol was carried out within five hours. Moreover, a bioinformatic background is not required due to the user-friendly interface of GENEIOUS as all steps of data processing are easy to execute and results are presented in a clear way as query-centric alignment. In addition, no highly complicated analysis procedure is needed as the nanopore data was used for identifying the serotype, not for whole genome assembly, which is more convoluted.

In this study, we applied a local BLAST search to align the obtained reads to FMDV sequences stored in GenBank. By establishment of an offline local data-calling algorithm, we decreased the time to result and overcame the need of a stable and powerful internet connection, which is usually absent in the areas most affected by FMD. The FMDV open reading frame consists of four functional regions (L, P1-3). P1 encodes the structural proteins of the virus, while L, P2 and P3 contain nonstructural proteins that play an important role in virulence, viral replication and virus-host interaction (Gao et al., 2016). It is not surprising that the P1 region worked best for serotyping because i) P2 and P3 are more conserved between the different serotypes ii) the NCBI database did not contain enough sequences of the P2 and P3 regions to cover all variants of the serotypes (Carrillo et al., 2005). Co-infection with different serotypes can be efficiently determined via the calculation of the number of hits allocated to each serotype.

Using sequencing overcomes the problem of variability in the genome as viruses from all serotypes can be detected independent of the geographical region or virus pool.

Previously published sequencing protocols rely mainly on sequencing by synthesis using either Sanger or Illumina sequencing (Knowles et al., 2016; Logan et al., 2014). These approaches are cost-effective and yield accurate sequences with high throughput. However, both methods are not field applicable, due to the need of expensive and complex devices, and have time-consuming protocols. Additionally, Illumina sequencing requires a bioinformatic background for data analysis. In contrast, the nanopore sequencing produced results in a very short time and the whole procedure can be performed in a mobile suitcase laboratory (Abd El Wahed et al., 2015), with no need for sophisticated infrastructure or laboratory capacities. This enables serotyping directly at the site of an outbreak in low-resource settings. In FMDV-free countries, native samples from suspect animals must be handled with greater care, but after they have been treated with a lysis buffer, the downstream analysis can be safely performed under BSL-2 conditions.

A $-20^{\circ} \mathrm{C}$ freezer is still needed for long-term storage of sequencing reagents. Fortunately, the sequencing kits and the flow cells can be kept at room temperature for one day, which is more than enough to perform the experiment. One of the big limitations of the technology is the cost per one sequencing run for seven samples $(€ 2300)$ and another $€ 8500$ is needed as a starting cost for the suitcase laboratory.

Rapid sequencing of RNA viruses was established before by others (Batovska et al., 2017) employing the SuperScript III First-Strand Synthesis System (ThermoFisher Scientific, Waltham, MA, USA) and random hexamer primers for cDNA synthesis along with the SQKNSK007 kit (Oxford Nanopore Technologies, Oxford, UK) for library preparation. By contrast, in our study we have used the SuperScript IV VILO kit, which produces higher quality cDNA in less time (10 min). In addition, the application of a poly-T primer binding to the poly-A tail of the FMDV RNA has allowed the synthesis of a long cDNA strand instead of multiple cDNA fragments in the case of random hexamer primers. Furthermore, the SQK-NSK007 kit is designed for the sequencing of a single sample, while the SQK-DCS 108 kit allowed us to sequence seven samples simultaneously in the same run.

Generally, various sequencing methods have a limit of detection at $10^{4}$ DNA molecules per tested sample (Liu et al., 2012). In clinical cases 
of FMDV, the viral load in the vesicular lesions is high $\left(\geq 10^{11}\right.$ genome copies $/ \mathrm{g}$ ) and in the preclinical phase it ranges from $10^{5.1}$ to $10^{9.8}$ genome copy/g (Pharo, 2002; Stenfeldt et al., 2015). In carrier animals, the viral load in probang samples is low: $10^{3.5}-10^{5.2} / \mathrm{ml}$ (Stenfeldt et al., 2016). However, the amount of highly purified RNA needed for sequencing can easily be obtained either directly or by a nucleic acid concentration method.

\section{Conclusions}

In conclusion, we have developed a fast and reliable method for sequence-based serotyping of FMDV. The whole procedure can be easily implemented in a regular laboratory workflow or with a mobile suitcase laboratory in a low-resource setting. The next points to address will be the decrease of pipetting steps and the switch to lyophilized reagents, which are stable at ambient temperature.

\section{Funding}

This work was supported by Alexander von Humboldt Foundation (3.4 - EGY/1026128). The supporter has no involvement in decisions concerning this article.

\section{Declarations of interest}

None.

\section{Acknowledgment}

The study was funded by Alexander von Humboldt Foundation (3.4 - EGY/1026128).

\section{Appendix A. Supplementary data}

Supplementary material related to this article can be found, in the online version, at doi:https://doi.org/10.1016/j.jviromet.2018.10.020.

\section{References}

Abd El Wahed, A., El-Deeb, A., El-Tholoth, M., El, Abd, Kader, H., Ahmed, A., Hassan, S., Hoffmann, B., Haas, B., Shalaby, M.A., Hufert, F.T., Weidmann, M., 2013. A portable reverse transcription recombinase polymerase amplification assay for rapid detection of foot-and-mouth disease virus. PLoS One 8, e71642.

Abd El Wahed, A., Weidmann, M., Hufert, F.T., 2015. Diagnostics-in-a-Suitcase: development of a portable and rapid assay for the detection of the emerging avian influenza A (H7N9) virus. J. Clin. Virol. 69, 16-21.

Bachanek-Bankowska, K., Mero, H.R., Wadsworth, J., Mioulet, V., Sallu, R., Belsham, G.J., Kasanga, C.J., Knowles, N.J., King, D.P., 2016. Development and evaluation of tailored specific real-time RT-PCR assays for detection of foot-and-mouth disease virus serotypes circulating in East Africa. J. Virol. Methods 237, 114-120.

Batovska, J., Lynch, S.E., Rodoni, B.C., Sawbridge, T.I., Cogan, N.O., 2017. Metagenomic arbovirus detection using MinION nanopore sequencing. J. Virol. Methods 249, 79-84.

Carrillo, C., Tulman, E.R., Delhon, G., Lu, Z., Carreno, A., Vagnozzi, A., Kutish, G.F., Rock, D.L., 2005. Comparative genomics of foot-and-mouth disease virus. J. Virol. 79 , 6487-6504.

Dill, V., Beer, M., Hoffmann, B., 2017. Simple, quick and cost-efficient: A universal RTPCR and sequencing strategy for genomic characterisation of foot-and-mouth disease viruses. J. Virol. Methods 246, 58-64.

Gao, Y., Sun, S.Q., Guo, H.C., 2016. Biological function of Foot-and-mouth disease virus non-structural proteins and non-coding elements. Virol. J. 13, 107.

Gullberg, M., Polacek, C., Botner, A., Belsham, G.J., 2013. Processing of the VP1/2A junction is not necessary for production of foot-and-mouth disease virus empty capsids and infectious viruses: characterization of "self-tagged" particles. J. Virol. 87, 11591-11603.

Knight-Jones, T.J., Rushton, J., 2013. The economic impacts of foot and mouth disease what are they, how big are they and where do they occur? Prev. Vet. Med. 112, 161-173.

Knowles, N.J., Wadsworth, J., Bachanek-Bankowska, K., King, D.P., 2016. VP1 sequencing protocol for foot and mouth disease virus molecular epidemiology. Rev. Sci. Tech. 35, 741-755.

Liu, L., Li, Y., Li, S., Hu, N., He, Y., Pong, R., Lin, D., Lu, L., Law, M., 2012. Comparison of next-generation sequencing systems. J. Biomed. Biotechnol., 2513642012.

Logan, G., Freimanis, G.L., King, D.J., Valdazo-Gonzalez, B., Bachanek-Bankowska, K., Sanderson, N.D., Knowles, N.J., King, D.P., Cottam, E.M., 2014. A universal protocol to generate consensus level genome sequences for foot-and-mouth disease virus and other positive-sense polyadenylated RNA viruses using the Illumina MiSeq. BMC Genom. 15, 828.

Longjam, N., Deb, R., Sarmah, A.K., Tayo, T., Awachat, V.B., Saxena, V.K., 2011. A brief review on diagnosis of foot-and-mouth disease of livestock: conventional to molecular tools. Vet. Med. Int., 9057682011.

Pharo, H.J., 2002. Foot-and-mouth disease: an assessment of the risks facing New Zealand. N. Z. Vet. J. 50, 46-55.

Roeder, P.L., Le Blanc Smith, P.M., 1987. Detection and typing of foot-and-mouth disease virus by enzyme-linked immunosorbent assay: a sensitive, rapid and reliable technique for primary diagnosis. Res. Vet. Sci. 43, 225-232.

Soltan, M.A., Negmaldin, A.H., El-Diasty, M.M., Mansour, S.M.G., Elbadry, M.A., Wilkes, R.P., 2017. Molecular characterization of circulating Foot and mouth disease virus (FMDV) serotype $\mathrm{O}$ topotype EA-3 and serotype A (African topotype) genotype IV in Egypt, 2016. Vet. Microbiol. 208, 89-93.

Stenfeldt, C., Eschbaumer, M., Pacheco, J.M., Rekant, S.I., Rodriguez, L.L., Arzt, J., 2015. Pathogenesis of primary foot-and-Mouth disease virus infection in the nasopharynx of vaccinated and non-vaccinated cattle. PLoS One 10, e0143666.

Stenfeldt, C., Eschbaumer, M., Rekant, S.I., Pacheco, J.M., Smoliga, G.R., Hartwig, E.J., Rodriguez, L.L., Arzt, J., 2016. The foot-and-Mouth disease carrier state divergence in cattle. J. Virol. 90, 6344-6364.

Tekleghiorghis, T., Weerdmeester, K., van Hemert-Kluitenberg, F., Moormann, R.J., Dekker, A., 2014. Comparison of test methodologies for foot-and-mouth disease virus serotype A vaccine matching. Clin. Vaccine Immunol. 21, 674-683.

Zhu, Y.Y., Machleder, E.M., Chenchik, A., Li, R., Siebert, P.D., 2001. Reverse transcriptase template switching: a SMART approach for full-length cDNA library construction. Biotechniques 30, 892-897. 


\section{Chapter V:}

\section{Diagnosing Zika virus infection against a background of other flaviviruses: Studies in high resolution serological analysis}

Sören Hansen ${ }^{1 \#}$, Sven-Kevin Hotop ${ }^{2 \& 3 \#}$, Oumar Faye ${ }^{4}$, Oumar Ndiaye ${ }^{4}$, Susanne Böhlken-Fascher ${ }^{1}$, Rodrigo Pessôa ${ }^{5}$, Frank Hufert ${ }^{6}$, Christiane Stahl-Hennig ${ }^{7}$, Ronald Frank $^{8}$, Claus-Peter Czerny ${ }^{1}$, Jonas Schmidt-Chanasit ${ }^{9 \& 10}$, Sabri S. Sanabani ${ }^{5}$, Amadou A. Sall ${ }^{4}$, Matthias Niedrig ${ }^{11}$, Mark Brönstrup ${ }^{2 \& 3}$, Hans-Joachim Fritz ${ }^{12 \pi}$, Ahmed Abd El Wahed ${ }^{11^{*}}$

1) Division of Microbiology and Animal Hygiene, University of Goettingen, 37075, Germany

2) Department of Chemical Biology, Helmholtz-Zentrum für Infektionsforschung, Inhoffenstrasse 7, 38124 Braunschweig, Germany

3) Deutsches Zentrum für Infektionsforschung (DZIF), Standort Hannover-Braunschweig, Germany

4) Institut Pasteur de Dakar, Dakar, Senegal

5) Laboratory of Dermatology and Immunodeficiencies, LIM-56, Department of Dermatology, Tropical Medicine Institute of São Paulo, University of São Paulo, São Paulo, Brazil

6) Institute of Microbiology and Virology, Brandenburg Medical School Fontane, Senftenberg, Germany

7) Deutsches Primatenzentrum GmbH, Leibniz-Institut für Primatenforschung, Unit of Infection Models, Göttingen, Germany

8) AIMS Scientific Products GmbH, Berlin, Germany

9) Bernhard Nocht Institute for Tropical Medicine, WHO Collaborating Centre for Arbovirus and Hemorrhagic Fever Reference and Research, Hamburg, Germany

10) German Centre for Infection Research (DZIF), partner site Hamburg-Luebeck-Borstel, Hamburg, Germany

11) Robert Koch Institut, Nordufer 20, 13353 Berlin, Germany

12) Akademie der Wissenschaften zu Göttingen, Theaterstraße 7, 37073 Göttingen, Germany

\#Equal contribution

"Joint senior authors

*Corresponding author 


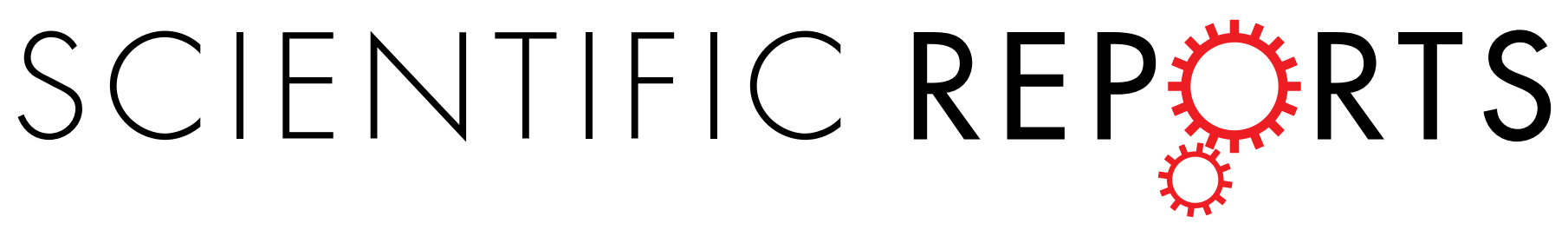

\title{
Diagnosing Zika virus infection against a background of other flaviviruses: Studies in high resolution serological analysis
}

\author{
Sören Hansen ${ }^{1}$, Sven-Kevin Hotop ${ }^{2,3}$, Oumar Faye ${ }^{4}$, Oumar Ndiaye ${ }^{4}$, Susanne Böhlken- \\ Fascher $^{1}$, Rodrigo Pessôa ${ }^{5}$, Frank Hufert ${ }^{6}$, Christiane Stahl-Hennig ${ }^{7}$, Ronald Frank ${ }^{8}$, \\ Claus-Peter Czerny ${ }^{1}$, Jonas Schmidt-Chanasit ${ }^{9}, 10$, Sabri S. Sanabani ${ }^{5}$, Amadou A. Sall ${ }^{4}$, \\ Matthias Niedrig ${ }^{11}$, Mark Brönstrup ${ }^{2,3}$, Hans-Joachim Fritz ${ }^{12}$ \& Ahmed Abd El Wahed ${ }^{1}$
}

Zika virus (ZIKV) is a mosquito-borne flavivirus. Homologous proteins of different flaviviruses display high degrees of sequence identity, especially within subgroups. This leads to extensive immunological cross-reactivity and corresponding problems for developing a ZIKV-specific serological assay. In this study, peptide microarrays were employed to identify individual ZIKV antibody targets with promise in differential diagnosis. A total of 1643 overlapping oligopeptides were synthesized and printed onto glass slides. Together, they encompass the full amino acid sequences of ZIKV proteomes of African, Brazilian, USA, and French Polynesian origins. The resulting ZIKV scanning microarray chips were used to screen three pools of sera from recent Zika outbreaks in Senegal and Cape Verde, in Brazil, and from overseas travelers returning to the EU. Together with a mixed pool of well characterized, archived sera of patients suffering from infections by dengue, yellow fever, tick-borne encephalitis, and West Nile viruses, a total of $\mathbf{4 2}$ sera went into the study. Sixty-eight antibody target regions were identified. Most of which were hitherto unknown. Alignments and sequence comparisons revealed 13 of which could be classified as bona fide ZIKV-specific. These identified antibody target regions constitute a founding set of analytical tools for serological discrimination of ZIKV from other flaviviruses.

Zika virus (ZIKV), a mosquito-borne flavivirus, was first isolated from sentinel Rhesus macaques in the Zika forest of Uganda in $1947^{1}$. The first human case was recorded in Nigeria in 1954. Before 2007, many small-scale epidemics were recorded in Africa and $\mathrm{Asia}^{2}$. Yap island (Micronesia) was the first area outside Africa and Asia to confirm human ZIKV infection ${ }^{3}$. Around one-tenth of the population of French Polynesia encountered the infection during the 2013 outbreak $^{4}$. In 2015, the Brazilian ministry of health estimated around 440,000 to 1,300,000 individuals in Brazil that may have contracted the infection. The potential of ZIKV spreading to other countries is high with almost one-fourth of the human population worldwide being at risk of encountering ZIKV infection. In regions like Europe, ZIKV transmission risk is present through high mobility and global connectivity ${ }^{5}$.

${ }^{1}$ Division of Microbiology and Animal Hygiene, University of Goettingen, Goettingen, 37075, Germany. ${ }^{2}$ Department of Chemical Biology, Helmholtz-Zentrum für Infektionsforschung, Inhoffenstrasse 7, 38124, Braunschweig, Germany. ${ }^{3}$ Deutsches Zentrum für Infektionsforschung (DZIF), Standort Hannover-, Braunschweig, Germany. ${ }^{4}$ Institut Pasteur de Dakar, Dakar, Senegal. ${ }^{5}$ Laboratory of Dermatology and Immunodeficiencies, LIM-56, Department of Dermatology, Tropical Medicine Institute of São Paulo, University of São Paulo, São Paulo, Brazil. ${ }^{6}$ Institute of Microbiology and Virology, Brandenburg Medical School Fontane, Senftenberg, Germany. ${ }^{7}$ Deutsches Primatenzentrum GmbH, Leibniz-Institut für Primatenforschung, Unit of Infection Models, Göttingen, Germany. ${ }^{8}$ AIMS Scientific Products GmbH, Berlin, Germany. ${ }^{9}$ Bernhard Nocht Institute for Tropical Medicine, WHO Collaborating Centre for Arbovirus and Hemorrhagic Fever Reference and Research, Hamburg, Germany. ${ }^{10}$ German Centre for Infection Research (DZIF), partner site Hamburg-Luebeck-Borstel, Hamburg, Germany. ${ }^{11}$ Robert Koch Institut, Nordufer 20, 13353, Berlin, Germany. ${ }^{12}$ Akademie der Wissenschaften zu Göttingen, Theaterstraße 7, 37073, Göttingen, Germany. Sören Hansen and Sven-Kevin Hotop contributed equally. Hans-Joachim Fritz and Ahmed Abd El Wahed jointly supervised this work. Correspondence and requests for materials should be addressed to A.A.E.W. (email: abdelwahed@gwdg.de) 
Clinical signs of ZIKV infection are ranging from asymptomatic to mild headaches, fever, skin rashes and joint pain, which usually last a few days. The symptoms are unspecific and are frequently misdiagnosed as caused by dengue or chikungunya virus ${ }^{6}$. ZIKV is transmitted mainly by Aedes mosquitoes. Many reports discuss two additional ZIKV infection pathways: sexual intercourse and from a pregnant woman to her fetus ${ }^{7,8}$. ZIKV RNA was detected in the amniotic fluid of a pregnant woman ${ }^{9}$ and in the brain of an aborted fetus afflicted with microcephaly $^{10}$. Moreover, ZIKV was linked to increased numbers of Guillian-Barré Syndrome cases in affected countries ${ }^{11}$.

ZIKV RNA can be detected in blood and saliva during the first five days of onset of symptoms, up to 21 days in urine and for up to one year in semen ${ }^{12-15}$. Longer persistence of anti-ZIKV antibodies in serum (IgM: up to two months; IgG: two years) ${ }^{16,17}$ extends the practicable diagnostic time window. On the other hand, high degrees of sequence identity of homologous proteins and concomitant immunological cross-reactivity among all flaviviruses, in particular within the various subgroups, have been reported ${ }^{18,19}$. These facts profoundly complicate serological diagnosis, especially in areas, where ZIKV and dengue virus are co-circulating and/or yellow fever vaccine is applied routinely ${ }^{20,21}$. Still, serological differentiation of ZIKV from other flaviviruses remains desirable and some progress towards resolving the difficulties has been announced ${ }^{22-24}$. This present study introduces a new approach resting on the identification, in larger numbers, of ZIKV-specific antibody targets. Specifically, we report comparative deconvolution of complex B cell responses against ZIKV and other flavivirus by screening corresponding sera with a microarray chip made up of overlapping peptides covering the entire amino acid sequence of the ZIKV genomic polyprotein. As a result, 13 short linear stretches of polypeptide sequence, scattered throughout the viral proteome, were identified that exhibit exclusive reactivity towards ZIKV antisera.

\section{Results}

A (series of) positive array spot(s) indicates an antibody target and positions it within a protein sequence. In the context of the overlapping peptides method, the term 'antibody target' denotes a stretch of polypeptide chain of an antigen encompassing either a single linear B cell epitope or a locally clustered set thereof. In the latter case, it is called an antibody target region (ATR $)^{25}$. Since a spots series as such often does not allow distinguishing between single epitope and ATR, the latter term is occasionally also used as a synonym of the generic name "antibody target" with epitopes regarded as extreme cases within that class.

Compiling a comprehensive list of antibody targets (epitopes, ATRs) participating in B cell responses to ZIKV infection and singling out from it the ZIKV-specific subset will, so the working hypothesis, enable diagnosis of ZIKV infection against a background of extensively cross-reacting other flaviviruses (e.g. dengue, yellow fever, tick-borne encephalitis, and West Nile virus). As shown earlier ${ }^{25}$, the necessary deconvolution of complex B cell responses to the level of contributions made by individual short stretches of polypeptide chain can be achieved experimentally by peptide microarray techniques. Comparing ATRs resulting from inspection of ZIKV and non-ZIKV flaviviral antisera will reduce the former to the ZIKV-specific subset. From this, discriminating immunochemical reagents can be derived.

Immunochemical experiments. Three pools of sera from ZIKV-infected individuals and one mixed pool of anti-flavivirus sera were prepared and screened for serological footprints (separate experiments for IgM and IgG) employing peptide microarray chips (Fig. 1). Two sets of original data are displayed in Fig. 2. Positively responding array spots (Fig. 1) delineate antibody targets (white and blue frames in Fig. 2).

ATRs identified: Overview and crude classification. The experiments uncovered 68 ATRs, most of which were hitherto unknown. Of these, 21 were recognized by both IgM and IgG, 30 by IgM alone and 17 by IgG alone (Figs 2-4 and Supplementary Table S1). The targets are scattered across the entire proteome with no striking preference of envelope over non-envelope proteins (Fig. 3). From antibodies directed against the latter, no contribution to virus neutralization would be expected, but in the context of diagnosis they are equally useful. Twenty-two ATRs reacted exclusively with ZIKV sera (Figs 4 and 5, classes I and II), 13 exclusively with FlaviMix (classes IV and V) and 33 with both FlaviMix and at least one ZIKV pool (class III). Sequences of corresponding stretches of polypeptide chain are compiled in Supplementary Tables S2 and S3. Alignments and sequence comparisons prompted sub-division of the ZIKV-exclusive and the FlaviMix-exclusive sets as explicated in detail below. Note that with "exclusive" we merely describe an observation in the present assay - in contrast to "specific" as an intrinsic property of a given antibody target.

Strings of amino acid residues displaying exclusive immunochemical reactivity towards ZIKV sera would be expected to differ substantially between ZIKV and the respective homologous sites of viruses making up the FlaviMix pool. This was true for 13 of 22 cases (class I, Supplementary Table S2, Fig. 5). Class I cases (such as ATR \#2062, listed in Table 1) are characterized by a low to intermediate level of sequence identity between ZIKV and other flaviviruses across the respective antigen (NS4 in the case of ATR \#2062) and a similarly low or lower degree of identity within the actual target region under consideration. The 13 class I ATRs are distributed among all ZIKV antigens - with the exception of antigens C and NS3 (Fig. 3). For the remaining nine cases (class II), sequence identity across the entire length of the respective antigen is low to intermediate but within the target region it is high between either ZIKV and all viruses of the FlaviMix pool (class IIa, five cases, represented in Table 1 by ATR \#388) or ZIKV and the various Dengue subtypes only (class IIb, four cases, represented in Table 1 by ATR \#2029).

There are thirteen ATRs reacting exclusively with the FlaviMix pool. Five of these (class IV: \#718, \#1024, \#1618, \#2836, \#3325, Supplementary Table S3) show strong similarity or identity with the respective homologous sites in ZIKV. Eight cases of FlaviMix-exclusive responses remain unexplained (class V: ATRs \#25, \#103, \#1441, \#1585, \#1741, \#1969, \#2308, \#2791, Supplementary Table S1). Anyhow, class V ATRs lack relevance to the present problem as they will not make any contribution to the development of a practical serological test. 


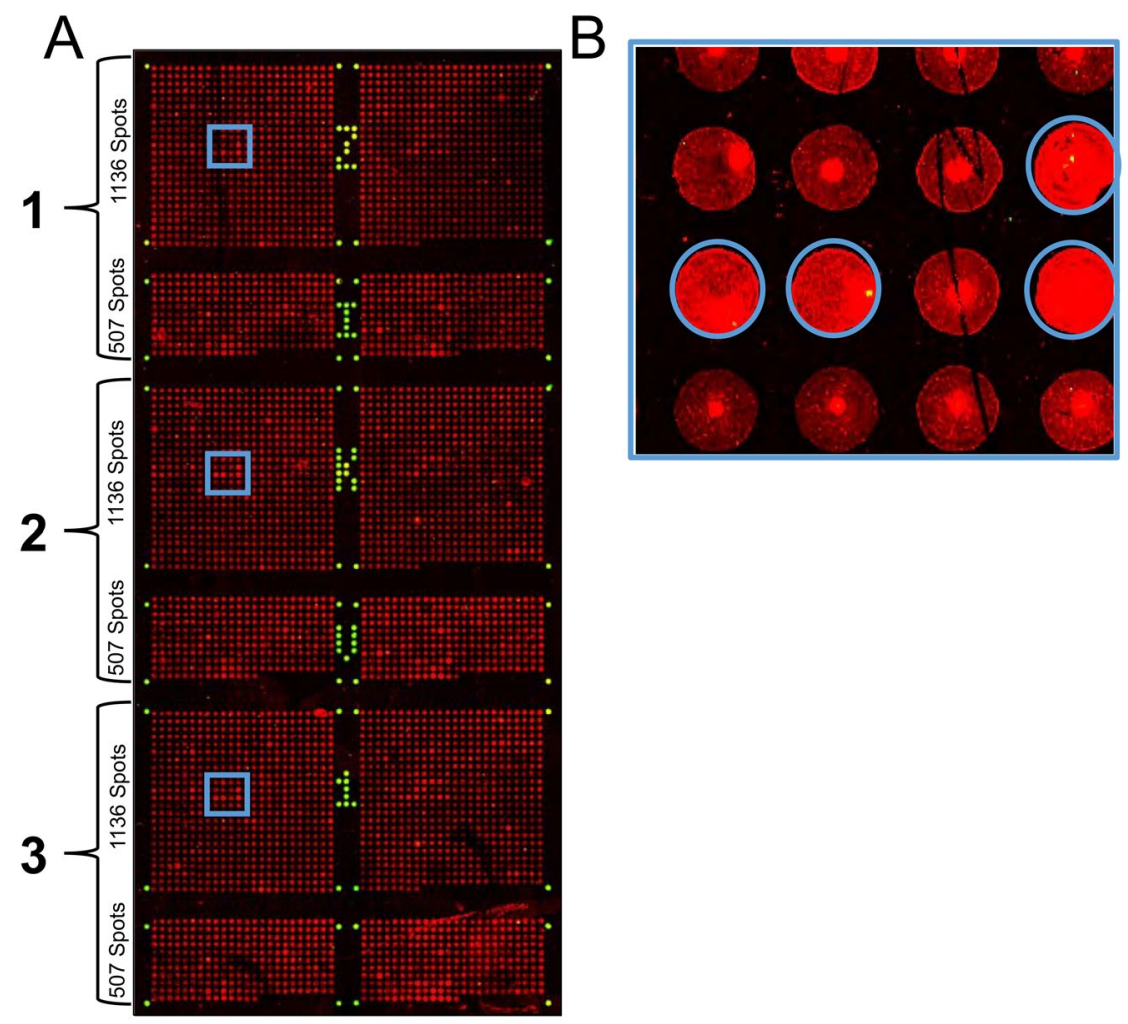

Figure 1. The ZIKV peptide microarray chip. (Panel A) Layout: Each chip hosts three identical arrays, each of which encompasses 1643 pentadekapeptides: 1136 covering the complete ZIKV Africa (AAV34151) polyprotein with an overlap of consecutive individual peptides of 12 amino acid residues plus 507 pentadekapeptides accommodating divergent sequences present in other ZIKV isolates (Brazil, USA, Senegal, French Polynesia). In addition, there are 96 biotin spots (green), which serve as internal positive controls and as markers of array boundaries (ref. ${ }^{23}$ ). (Panel B) Identification of positive signals: The criterion for a positive response is bright red fluorescence spread evenly across the entire spot area (examples marked by a blue circle).

\section{Discussion}

This work marks the entry into a systematic and comprehensive search for antibody targets (epitopes, ATRs) of ZIKV in relation to those of other flaviviruses with the aim of identifying diagnostically useful antigenic sites eliciting ZIKV-specific antibodies. Despite the limited number of sera available for our analyses, some remarkable reactivity patterns emerged.

Antibody targets reacting with ZIKV sera pools exclusively. Among the 68 ATRs, 22 (summarized in Supplementary Table S2) reacted exclusively with one or more ZIKV sera pools, 13 recognized by IgM and 9 by IgG with no overlap of the two sets. This lack of overlap is surprising in view of the fact that the combined processes of affinity maturation and class-switching do not qualitatively change the antigen recognition properties of an antibody: In a notional, infinitely large collection of ZIKV-positive sera, all antibody targets should be represented in both the IgM and the IgG set.

High local sequence conservation of an antigenic site across various virus species immediately suggests cross-reactivity. As cross-reactivity was missing in some cases, an explanation could, in principle, be offered by different antigenicity of the same stretch of polypeptide chain in homologous antigens due to different local three-dimensional structure (high for ZIKV, low for other flaviviruses). Some differences of 3D-structure have indeed been observed among different flaviviruses ${ }^{26}$.

Taken together, the findings corroborate the validity of the rationale underlying this study and the thirteen class I ATRs are the first bona fide candidates for being truly ZIKV-specific as required for serologically diagnosing ZIKV infection against a dense background of cross-reactivity with other flaviviruses.

Cross-reacting antibody targets. Expectations that close serological scrutiny would reveal extensive cross-reactivity between ZIKV and other flaviviruses were borne out: At least 33 of the 68 ATRs identified in ZIKV and FlaviMix cross-react. As argued below, the real number is probably as high as 47 (Fig. 5). All ZIKV antigens carry a multitude of ATRs eliciting cross-reacting antibodies (Fig. 3) - a serious handicap for any serological ZIKV assay resting on whole antigens. Nevertheless, at least one attempt based on whole NS1 antigens of ZIKV and DENV (three cross-reacting ATRs by our count) has been made employing a combination of tests and quantitative signal comparison ${ }^{23}$. Prekumar et al. alleviate the cross-reactivity problem by focussing on two isolated domains of the ZIKV envelope protein that combine pronounced antigenicity with low sequence 


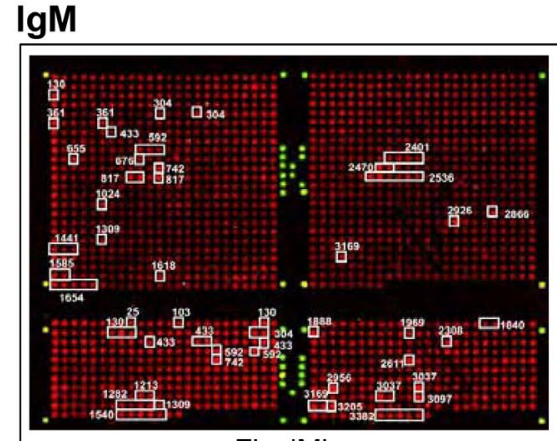

FlaviMix

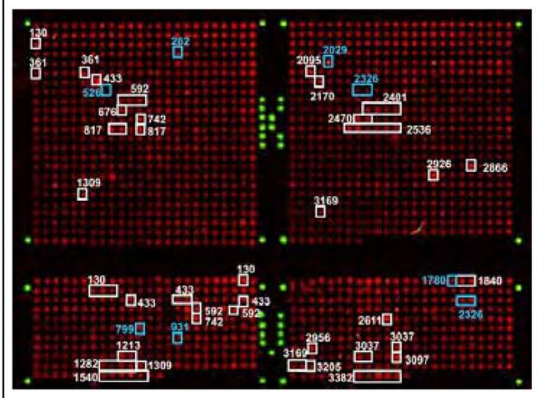

Africa

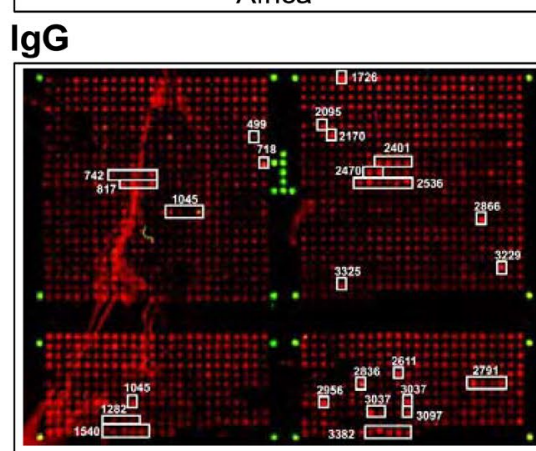

Flavimix

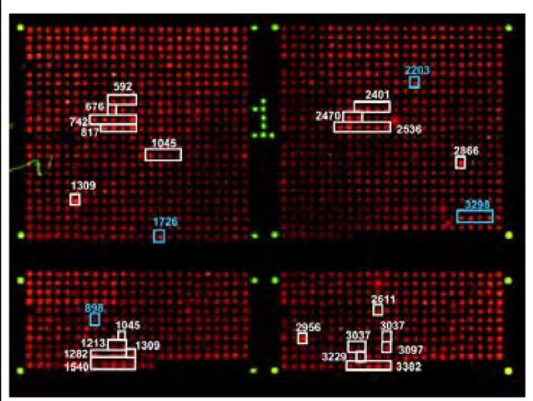

Africa

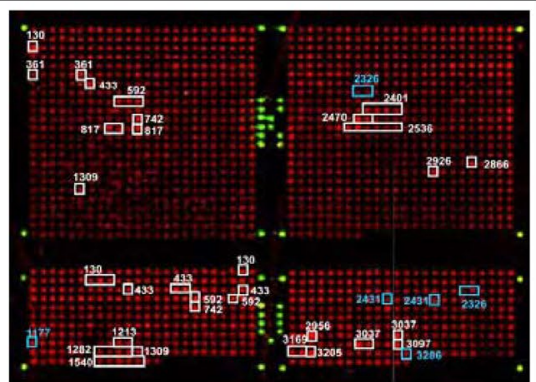

EU

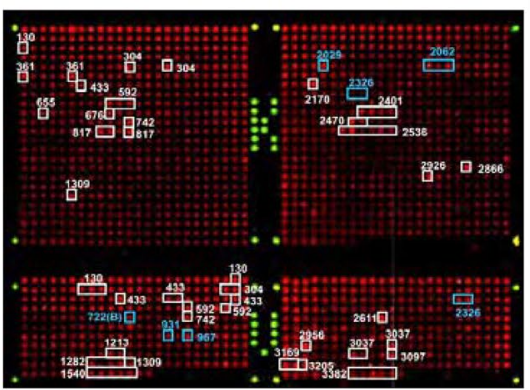

Brazil

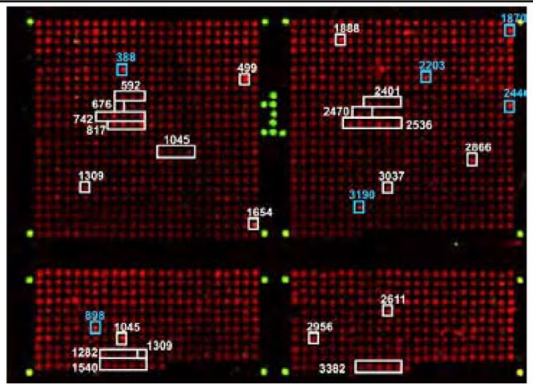

EU

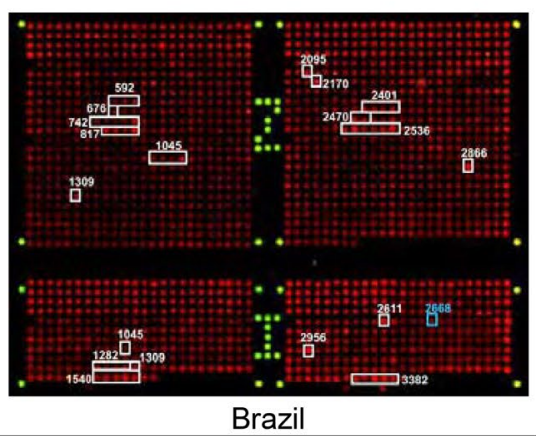

Figure 2. Original screening data (examples). Array spots showing positive reaction are boxed in. White boxes: Responses seen with FlaviMix pool (either exclusive or in combination with one or several ZIKV serum pools). Blue boxes: Responses seen only with ZIKV serum pools (“ZIKV-exclusives"). ATR numbers are stated next to corresponding boxes (Fig. 3). For experimental details refer to Materials and Methods.

similarity between ZIKV and DENV ${ }^{22}$. Lastly, a competition ELISA based on a ZIKV-specific monoclonal anti-NS1 antibody has shown remarkable selectivity and specificity ${ }^{21}$. The assay, however, is bound to miss detecting ZIKV-infections of individuals that happened not to produce antibodies against that one particular epitope.

Experimental results compared with epitope prediction. Two studies have been published that aim at predicting B cell epitopes of ZIKV envelope proteins on the basis of theoretical considerations and homology to other flaviviruses ${ }^{27,28}$. Our experiments confirm 18 of these predictions and uncovered 37 additional targets (classes I to III, Fig. 5), not only in non-envelope proteins. On the other hand, 28 predictions could not be verified 


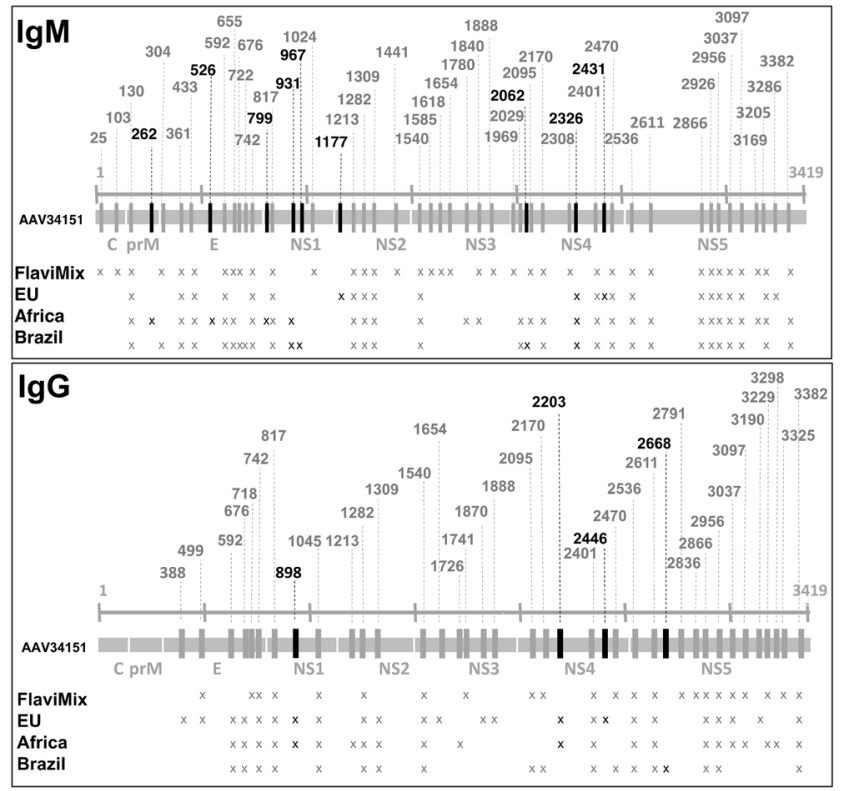

Figure 3. Mapping ATRs to ZIKV polyprotein. The horizontal bar represents the 3419 residue long unprocessed translation product of the ZIKV genome (Genbank accession number AAV34151). Marks along the grey line are spaced in intervals of 500 amino acid residues. ATRs are named by the number of their residue lying most closely to the N-terminus (Supplementary Table S1). ATRs marked black (class I ATRs, Fig. 5 and Supplementary Table S2) exclusively reacted with one or more ZIKV pools. ATRs marked grey reacted with FlaviMix pool and with zero to three ZIKV pools.

experimentally. This, however, does not necessarily indicate failed predictions but could alternatively be due, as in cases mentioned above, to a still incomplete collection of experimental targets. For a comprehensive comparison of prediction and experiment refer to Supplementary Table S4.

Non-saturating sampling of antibody targets. The notion of incomplete experimental representation of ZIKV ATRs in a limited number of human sera turned up in several different contexts. These are as follows. (i) Nine ATRs (class II) responded only to ZIKV sera despite their occurrence in homologous FlaviMix antigens with identical or very similar sequences. (ii) Reciprocally: Five ATRs of high sequence similarity between ZIKV and other flaviviruses (class IV) reacted exclusively with the FlaviMix pool. (iii) Among the 22 ZIKV-exclusive responses there is no overlap between ATRs recognized by the IgM and the IgG set of antibodies. (iv) Only a subset of epitopes, previously predicted on theoretical grounds ${ }^{27,28}$, could be verified (so far) experimentally.

For every individual one of these four independent observations, incomplete ATR sampling offers an only tentative explanation; the notion, however, gains cogency by giving all of them a common, unified underpinning. As a corollary, two predictions follow: (a) Very likely, class II and class IV ATRs are true cross-reactors (Fig. 5). In contrast, there is no reason a priori to doubt the ZIKV specificity of the 13 members of class I. (b) A fair number of ZIKV ATRs are still to be discovered. This may turn out to be of practical importance in the eventual compilation of a larger set of ZIKV-specific ATRs.

\section{Conclusion and Outlook}

Specific serological identification of ZIKV against a background of cross-reacting other flaviviruses requires use of ZIKV-specific antibody targets. Since, in principle, any single target is represented in an individual B cell response with a probability of less than one, high sensitivity standards can only be met by the combined application of several targets in parallel - each characterized by highest possible antigenicity. In that sense, substantial headway towards the development of a ZIKV-specific diagnostic assay has been made with the discovery of 13 bona fide ZIKV-specific ATRs as reported here.

The remaining agenda en route to a practical diagnostic procedure such as ELISA, line assay, or bead flow-through assay is as follows. (i) Still other potentially cross-reacting flaviviruses (e.g. Japanese encephalitis and spondweni viruses) need to be included. (ii) The assignment of the 13 ATRs in class I as ZIKV-specific needs to be further consolidated. (iii) Additional specific ATRs should be found and ranked with respect to their antigenicity (i.e. signal frequency in a set of test sera).

All of the above points can be addressed in a straightforward manner, larger numbers of sera being the only prerequisite lying outside the scope of the present study. The approach builds upon antigen amino acid sequence as the only indispensable knowledge and it is not limited to addressing the cross-reactivity problem of flaviviruses only.

Beyond diagnosis, peptide microarray-based, high resolution serology may contribute to gaining a better understanding of a number of infection-connected problems. Antibody-mediated virus enhancement, for example, is a well-documented phenomenon associated with flaviviruses and epidemiological studies linked Zika 


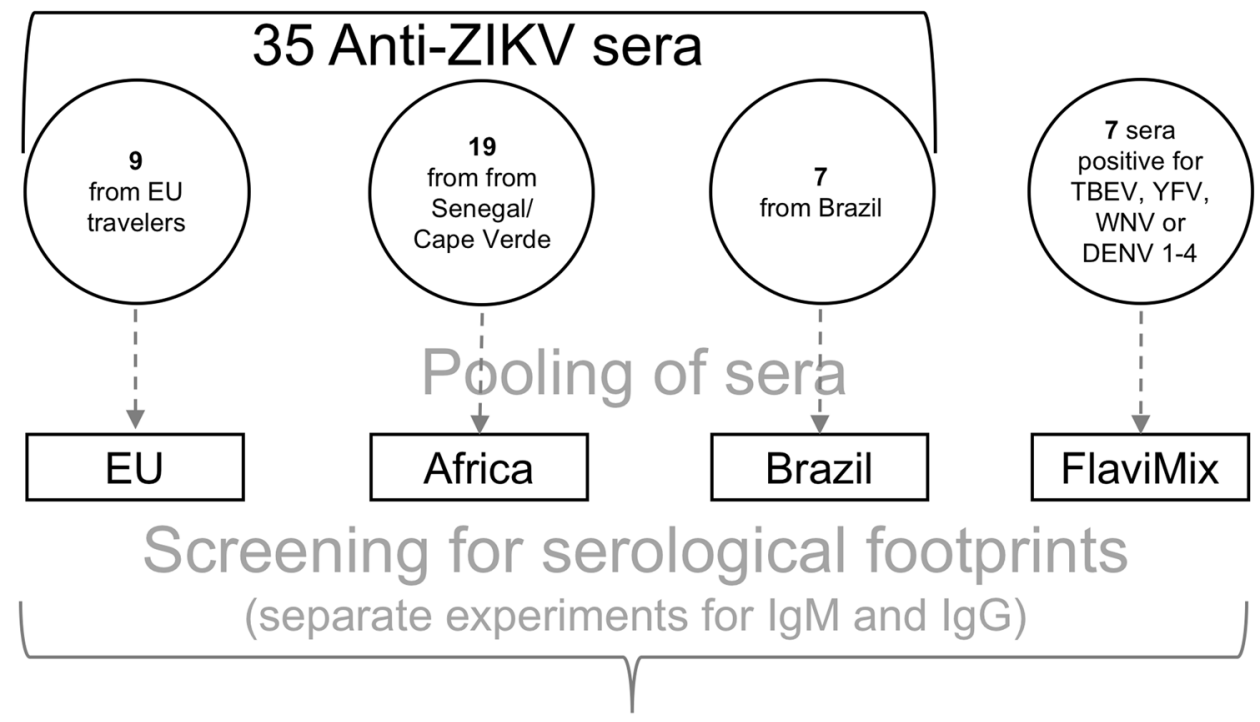

\section{ATRs}

( 21 recognized by both $\lg M$ and $\lg G, 30$ by $\lg M$ alone and 17 by $\lg G$ alone)

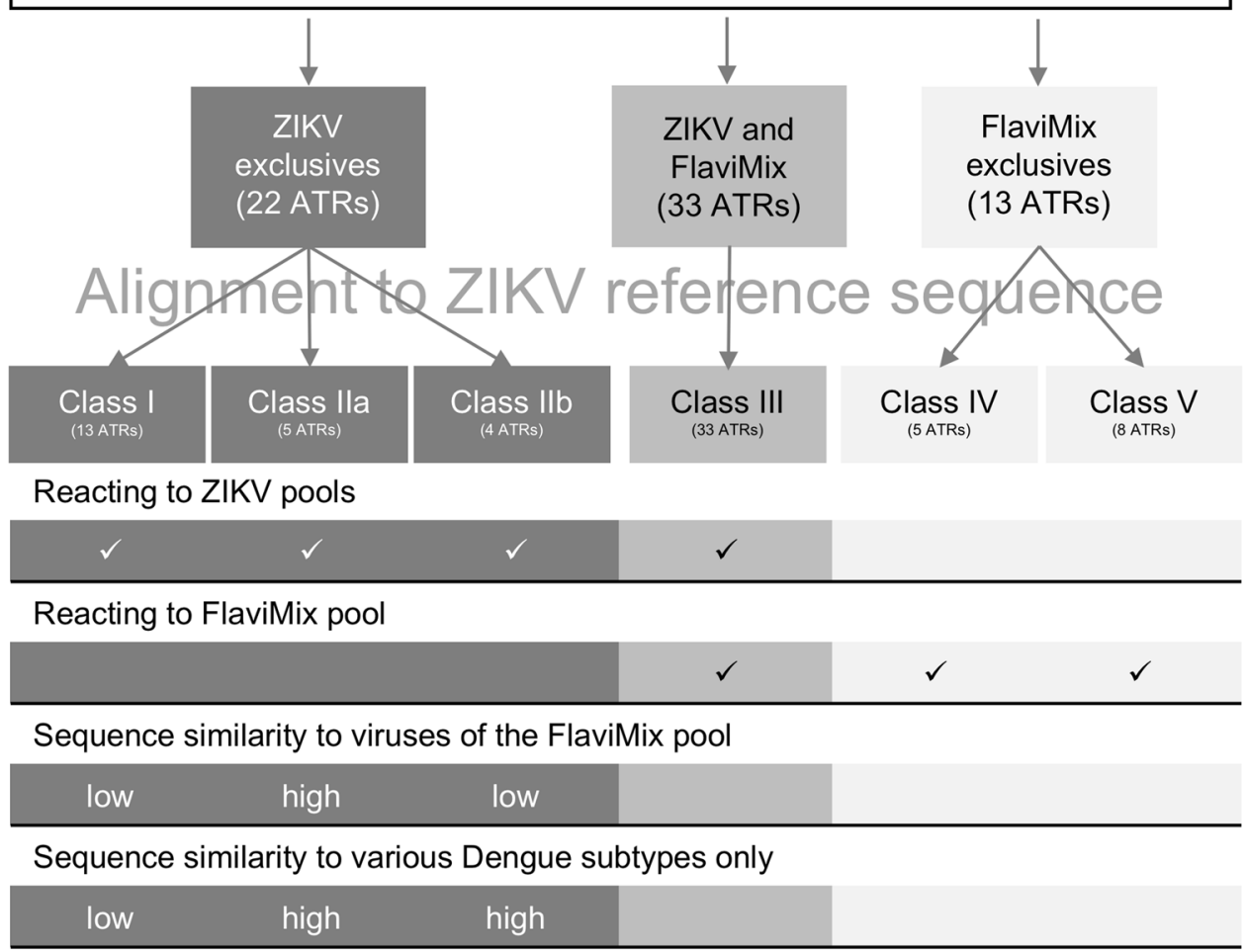

High Similarity between ZIKV and one or all FlaviMix viruses

Figure 4. Workflow of the study.

outbreaks to increased incidence of Guillain-Barré syndrome, a neurological autoimmune condition known to interact with humoral immunity ${ }^{29}$.

\section{Materials and Methods}

Serum samples and ethics statement. Samples of 42 well-characterized sera were provided by reference laboratories from Germany (Bernhard Nocht Institute, Hamburg), Senegal (Institut Pasteur de Dakar) and Brazil (University of São Paulo). The FlaviMix sera pool consists of 7 sera directed against dengue 1-4, yellow fever, tickborne encephalitis and West Nile viruses. The EU pool comprises 9 anti-ZIKV sera from EU travellers returning from Brazil. The Africa pool encompasses 19 anti-ZIKV sera from patients living in Senegal and Cape Verde. The 


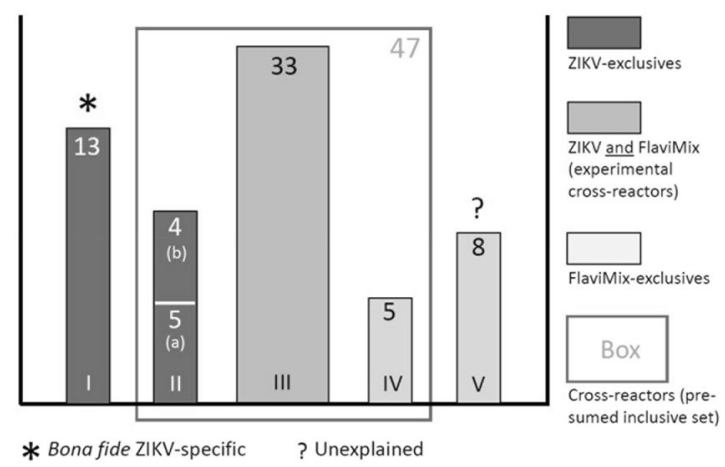

Figure 5. Classification of 68 experimentally identified ATRs. Roman numerals: Name of class. Arabic numerals: Number of ATRs in respective class. Class I: ZIKV-exclusives with low similarity to homologous sequences of FlaviMix viruses (residue identity $<80 \%$ ). Class II: Same with high similarity (residue identity $\geq 80 \%$ ). IIa: Similarity present in sequences of all viruses making up FlaviMix pool. IIb: Same but similarity only observed for Dengue subtypes. Class III: ATRs eliciting antibodies responding to both ZIKV and FlaviMix sera pools. Class IV: FlaviMix-exclusives with high similarity between ZIKV and FlaviMix. Class V: FlaviMixexclusives with no clear-cut location on polyprotein map of any virus represented in FlaviMix. Numbers of cases are graphically represented as bar areas. For usage of the term "exclusive" refer to main text.

Brazilian pool contains 7 anti-ZIKV sera from patients diagnosed at the University of São Paulo. All sera were either reference sera from the WHO collaboration center (Institut Pasteur de Dakar) or collected from patients during routine medical examinations or surveillance. All samples were collected between 5 to 28 days post onset of fever from patients tested positive with real-time PCR to the respective virus during the acute phase ( $>5$ days). Samples which reacted positive to more than one virus were excluded from the study. Patients had given consent according to national and international ethical regulations. All samples were handled anonymously.

ZIKV peptide microarray scanning chips. The ZIKV proteome of the African strain is made up of seven antigens (prM not counted as a separate module) with a cumulative length of 3419 amino acid residues ${ }^{30}$. Antigen sequences were broken up into 1136 pentdecapeptides with a consecutive overlap of 12 residues representing the ZIKV African strain (GenBank accession number AAV34151). These were synthesized via SPOT synthesis ${ }^{31}$, passed through the SC2 process $^{32}$ and spotted onto glass microscope slides (AIMS Scientific Products GmbH, Berlin, Germany) as illustrated in Fig. 1A (upper part of each of three identical arrays displayed in Fig. 1A). Each of the (smaller) lower array parts accommodates 507 additional pentadecapeptides that cover sequence stretches differing from the reference sequence in other ZIKV strains (Brazil, ALU33341; USA, AOS90225; Senegal, AHL43504; French Polynesia, AHZ13508). In addition, 96 spots of a biotin/( $\beta$-alanine $)_{2} /$ cellulose conjugate were deposited on the chips as positive controls and for marking array boundaries. After staining, they light up green (Fig. 1A).

Chip screening procedure. In each individual experiment, a ZIKV microarray chip was washed with absolute ethanol for $3 \mathrm{~min}$, then three times for $3 \mathrm{~min}$ each with TBS $(50 \mathrm{mM}$ Tris base, $150 \mathrm{mM} \mathrm{NaCl}, \mathrm{HCl}$ ad pH 7.6). After incubation with blocking buffer (TBS, $2 \%$ casein, $0.1 \%$ Tween-20) overnight, the chip was washed with T-TBS (TBS, $0.1 \%$ Tween-20) for $3 \mathrm{~min}$. Thereafter, $70 \mu \mathrm{l}$ of any of the four serum pools, diluted 120 -fold, were added onto the chip. The chip was incubated in a humidified chamber at $4^{\circ} \mathrm{C}$ overnight. Thereafter, the chip was washed three times for $5 \mathrm{~min}$ each with T-TBS. To visualize the binding of primary antibodies, the following was added onto the chip: $60 \mu \mathrm{l}$ of blocking buffer containing Cy3-conjugated mouse anti-biotin and Alexa Fluor 647-conjugated goat antibody directed against either human IgG or human IgM (240-fold dilution of stock solutions prepared according to instructions of the commercial supplier, Jackson Immunoresearch Laboratories, West Grove, PA, USA). The chip was kept in a humidified chamber at room temperature for 1.5 hours. Subsequently, the chip was washed twice for 5 min with T-TBS, three times with distilled water for 5 min each, and dried by dipping into acetonitrile for $10 \mathrm{sec}$ before visualization with an Agilent DNA microarray scanner. Each serum pool was tested in triplicate (three individual chips, corresponding to nine arrays total). For unknown reasons, all four sera pools produced atypically high levels of background which precluded application of the previously developed, automated spot calling procedure ${ }^{25}$. Therefore, array patterns were visually inspected and positive responses were identified in two steps - with the burden of safeguarding against false positives laid predominantly on stringent selection in step 1: (i) Individual signals were called positive if they showed bright red fluorescence spread uniformly across the entire spot area (Fig. 1B). (ii) A spot was taken as indicating an antibody target, if it passed step 1 in at least two arrays of the same chip with at least two out of three separate chips.

Sequence alignment. Bioinformatics: Experimentally determined ATRs were aligned to viral polyproteins using the pairwise alignment tool of the GENEIOUS program package (Biomatters Ltd, Auckland, New Zealand). Query sequences were composed of the respective ATR plus fifty amino acid residues each on both sides - all taken from the reference ZIKV strain (GenBank accession number: AAV34151). 


\begin{tabular}{|c|c|c|c|c|c|c|}
\hline ATR and Class & Virus (accession number) & \begin{tabular}{l|} 
Id (\%) Polyprotein \\
(within Subgroup)
\end{tabular} & Sequence & $\begin{array}{l}\text { Id (\%) } \\
\text { ATR }\end{array}$ & $\begin{array}{l}\text { Id (\%) } \\
\text { Antig. }\end{array}$ & $\begin{array}{l}\text { Id (\%) Antig. } \\
\text { within Subgroup }\end{array}$ \\
\hline \multirow{12}{*}{$\begin{array}{l}\text { ATR \#2062 (NS4) } \\
\text { [Class I: } 13 \text { members] } \\
\text { IgM (e,a,B) IgG }(e, a, b)\end{array}$} & Zika (AAV34151, Africa) & 100 & DGTTNNTIMEDSVP-AEVWTKY & 100 & 100 & \\
\hline & Zika (AOS90225, USA) & 96.4 & $\ldots \ldots \ldots \ldots \ldots-\ldots \ldots R H$ & 100 & 96.5 & \\
\hline & Zika (ALU33341, Brazil) & 96.4 & $\ldots \ldots \ldots \ldots \ldots-\ldots \ldots R H$ & 90.9 & 97.1 & \\
\hline & Zika (AHZ13508, Fr. Polynesia) & 96.5 & $\ldots \ldots \ldots \ldots \ldots-\ldots R H$ & 90.9 & 96.6 & \\
\hline & Zika (AHL43504, Senegal) & 99.0 & $\ldots \ldots \ldots \ldots \ldots-\ldots \ldots$ & 90.9 & 99.1 & \\
\hline & Dengue 1 (NP_059433) & $55.5(98.6)$ & ..ER..QVL.ENMD-V.I...E & 40.9 & 45.8 & 98.9 \\
\hline & Dengue 2 (NP_056776) & $55.5(97.7)$ & ..VK..Q.L.EN.E-V.I...E & 50.0 & 49.3 & 98.1 \\
\hline & Dengue 3 (YP_001621843) & $56.2(98.7)$ & ..QR..Q.L.ENMD-V.I...E & 45.5 & 47.0 & 98.1 \\
\hline & Dengue 4 (NP_073286) & $56.3(98.3)$ & T.ER..Q.L.ENME-V.I..RE & 36.4 & 47.3 & 98.8 \\
\hline & Yellow Fever (NP_041726) & $46.6(96.4)$ & E.PEEHE.LN. .GETVKCRAPG & 22.7 & 34.4 & 96.3 \\
\hline & West-Nile (YP_001527877) & $57.3(98.6)$ & ..PRT ...L..NNE-V . I. . L & 50.0 & 43.0 & 98.2 \\
\hline & TBE (NP_043135) & $41.5(95.8)$ & E.PEA.AVD.A.GDLVTFRSPN & 22.7 & 29.5 & 96.1 \\
\hline \multirow{12}{*}{$\begin{array}{l}\text { ATR \#388 (E) [Class } \\
\text { IIa: } 5 \text { members] IgM } \\
(\mathrm{e}, \mathrm{a}, \mathrm{b}) \operatorname{IgG}(\mathrm{E}, \mathrm{a}, \mathrm{b})\end{array}$} & Zika (AAV34151, Africa) & 100 & DRGWGNGCGLFGKGS & 100 & 100 & \\
\hline & Zika (AOS90225, USA) & 96.4 & $\ldots \ldots \ldots \ldots \ldots$ & 100 & 96.4 & \\
\hline & Zika (ALU33341, Brazil) & 96.4 & $\ldots \ldots \ldots \ldots$ & 100 & 96.2 & \\
\hline & Zika (AHZ13508, Fr. Polynesia) & 96.5 & $\ldots \ldots \ldots \ldots \ldots$ & 100 & 94.4 & \\
\hline & Zika (AHL43504, Senegal) & 99.0 & $\ldots \ldots \ldots \ldots \ldots$ & 100 & 98.9 & \\
\hline & \begin{tabular}{|l|} 
Dengue 1 (NP_059433) \\
\end{tabular} & $55.5(98.6)$ & $\ldots \ldots \ldots \ldots \ldots$ & 100 & 58.9 & 98.5 \\
\hline & Dengue 2 (NP_056776) & $55.5(97.7)$ & $\ldots \ldots \ldots \ldots G$ & 93.3 & 54.5 & 98.0 \\
\hline & Dengue 3(YP_001621843) & $56.2(98.7)$ & $\ldots \ldots \ldots \ldots$ & 100 & 58.7 & 98.5 \\
\hline & Dengue 4 (NP_073286) & $56.3(98.3)$ & $\ldots \ldots \ldots \ldots G$ & 93.3 & 56.7 & 98.3 \\
\hline & Yellow Fever (NP_041726) & $46.6(96.4)$ & $\ldots \ldots \ldots \ldots$ & 100 & 43.3 & 96.9 \\
\hline & West-Nile (YP_001527877) & $57.3(98.6)$ & $\ldots \ldots \ldots \ldots$ & 100 & 54.0 & 98.9 \\
\hline & TBE (NP_043135) & $41.5(95.8)$ & $\ldots \ldots$ н....... & 93.3 & 39.1 & 98.8 \\
\hline \multirow{12}{*}{$\begin{array}{l}\text { ATR \#2029 (NS4) } \\
\text { [Class IIb: } 4 \text { members] } \\
\text { IgM }(e, A, B) \text { IgG }(e, a, b)\end{array}$} & Zika (AAV34151, Africa) & 100 & TFVELMKRGDLPVWL & 100 & 100 & \\
\hline & Zika (AOS90225, USA) & 96.4 & $\ldots \ldots \ldots \ldots$ & 100 & 96.5 & \\
\hline & Zika (ALU33341, Brazil) & 96.4 & $\ldots \ldots \ldots \ldots \ldots$ & 100 & 97.1 & \\
\hline & Zika (AHZ13508, Fr. Polynesia) & 96.5 & $\ldots \ldots \ldots \ldots \ldots$ & 100 & 96.6 & \\
\hline & Zika (AHL43504, Senegal) & 99.0 & $\ldots \ldots \ldots \ldots \ldots$ & 100 & 99.1 & \\
\hline & Dengue 1 (NP_059433) & $55.5(98.6)$ & $\ldots \ldots R \ldots . . .$. & 93.3 & 45.8 & 98.9 \\
\hline & \begin{tabular}{|l|} 
Dengue 2 (NP_056776) \\
\end{tabular} & $55.5(97.7)$ & ... D.R...... & 86.7 & 49.3 & 98.1 \\
\hline & Dengue 3 (YP_001621843) & $56.2(98.7)$ & $\ldots \ldots R \ldots \ldots$ & 93.3 & 47.0 & 98.1 \\
\hline & Dengue 4 (NP_073286) & $56.3(98.3)$ & $\ldots \ldots R \ldots . . .$. & 93.3 & 47.3 & 98.8 \\
\hline & Yellow Fever (NP_041726) & $46.6(96.4)$ & V.R. .VRNC...... & 60.0 & 34.4 & 96.3 \\
\hline & West-Nile (YP_001527877) & $57.3(98.6)$ & N.L..LRTA...... & 60.0 & 43.0 & 98.2 \\
\hline & TBE (NP_043135) & $41.5(95.8)$ & H.RH.LTHC.FTP.. & 33.3 & 29.5 & 96.1 \\
\hline
\end{tabular}

Table 1. Properties of ZIKV-exclusive ATRs. Three ATRs exclusively responding to ZIKV sera are illustrated, one representative case of each class I, IIa and IIb (for classification refer to Fig. 5; a comprehensive compilation of all ZIKV-exclusive ATRs and their sequence characteristics is given in Supplementary Table S2). Id values, if not stated otherwise, denote \% sequence identity with reference strain Zika (AAV34151, Africa) of genomic polyprotein, respective antigen and ATR as indicated in column headers. In addition, Id values indicating bandwidths of sequence variation within subgroups of non-ZIKV flaviviruses (polyprotein or individual antigen) are listed. These were derived as follows. (i) A consensus sequence was computed for each subgroup. (ii) For each member of the respective subgroup percent residue identity with the consensus sequence was calculated. (iii) The mean value of these was defined as "Id within subgroup". Alignments were performed as described in Materials and Methods. Symbols for provenances of ZIKV-sera are as follows. E: EU pool, A: African pool, B: Brazilian pool. Capital letters (E, A, B) indicate positive signal observed with respective sera pool, while small letters (e, a, b) represent lack of reactivity with either IgM or IgG.

Ethical statement. No ethical approval was required as all sera were either reference sera from the WHO collaboration center (Institut Pasteur de Dakar) or collected from patients during routine medical examinations or surveillance. All samples were handled anonymously.

\section{Data Availability}

All data produced during this study were included in the manuscript. Original data files are available upon request to the corresponding author. 


\section{References}

1. Dick, G. W., Kitchen, S. F. \& Haddow, A. J. Zika virus. I. Isolations and serological specificity. Transactions of the Royal Society of Tropical Medicine and Hygiene 46, 509-520 (1952).

2. Singh, R. K. et al. Zika Virus - Emergence, evolution, pathology, diagnosis and control: current global scenario and future perspectives - A comprehensive review. Vet Q, 1-43, https://doi.org/10.1080/01652176.2016.1188333 (2016).

3. Duffy, M. R. et al. Zika virus outbreak on Yap Island, Federated States of Micronesia. The New England journal of medicine 360, 2536-2543, https://doi.org/10.1056/NEJMoa0805715 (2009).

4. Jouannic, J. M., Friszer, S., Leparc-Goffart, I., Garel, C. \& Eyrolle-Guignot, D. Zika virus infection in French Polynesia. Lancet 387, 1051-1052, https://doi.org/10.1016/S0140-6736(16)00625-5 (2016).

5. Samy, A. M., Thomas, S. M., Wahed, A. A., Cohoon, K. P. \& Peterson, A. T. Mapping the global geographic potential of Zika virus spread. Memorias do Instituto Oswaldo Cruz 111, 559-560, https://doi.org/10.1590/0074-02760160149 (2016).

6. Waggoner, J. J. \& Pinsky, B. A. Zika Virus: Diagnostics for an Emerging Pandemic Threat. J Clin Microbiol 54, 860-867, https://doi. org/10.1128/JCM.00279-16(2016).

7. D'Ortenzio, E. et al. Evidence of Sexual Transmission of Zika Virus. The New England journal of medicine, https://doi.org/10.1056/ NEJMc1604449 (2016).

8. Moron, A. F. et al. Microcephaly associated with maternal Zika virus infection. BJOG, https://doi.org/10.1111/1471-0528.14072 (2016).

9. Calvet, G. et al. Detection and sequencing of Zika virus from amniotic fluid of fetuses with microcephaly in Brazil: a case study. Lancet Infect Dis, https://doi.org/10.1016/S1473-3099(16)00095-5 (2016).

10. Mlakar, J. et al. Zika Virus Associated with Microcephaly. The New England journal of medicine 374, 951-958, https://doi. org/10.1056/NEJMoa1600651 (2016).

11. Brasil, P. et al. Guillain-Barre syndrome associated with Zika virus infection. Lancet 387, 1482, https://doi.org/10.1016/S01406736(16)30058-7 (2016).

12. Bingham, A. M. et al. Comparison of Test Results for Zika Virus RNA in Urine, Serum, and Saliva Specimens from Persons with Travel-Associated Zika Virus Disease - Florida, 2016. MMWR Morb Mortal Wkly Rep 65, 475-478, https://doi.org/10.15585/mmwr. mm6518e2 (2016).

13. Gourinat, A. C., O'Connor, O., Calvez, E., Goarant, C. \& Dupont-Rouzeyrol, M. Detection of Zika virus in urine. Emerging infectious diseases 21, 84-86, https://doi.org/10.3201/eid2101.140894 (2015).

14. Paz-Bailey, G. et al. Persistence of Zika Virus in Body Fluids - Preliminary Report. The New England journal of medicine, https://doi. org/10.1056/NEJMoa1613108 (2017).

15. Barzon, L. et al. Virus and Antibody Dynamics in Travelers With Acute Zika Virus Infection. Clinical infectious diseases: an official publication of the Infectious Diseases Society of America, https://doi.org/10.1093/cid/cix967 (2017).

16. Froeschl, G. et al. Long-term kinetics of Zika virus RNA and antibodies in body fluids of a vasectomized traveller returning from Martinique: a case report. BMC infectious diseases 17, 55, https://doi.org/10.1186/s12879-016-2123-9 (2017).

17. Rabe, I. B. et al. Interim Guidance for Interpretation of Zika Virus Antibody Test Results. MMWR Morb Mortal Wkly Rep 65, 543-546, https://doi.org/10.15585/mmwr.mm6521e1 (2016).

18. Heinz, F. X. \& Stiasny, K. The Antigenic Structure of Zika Virus and Its Relation to Other Flaviviruses: Implications for Infection and Immunoprophylaxis. Microbiol Mol Biol Rev 81, https://doi.org/10.1128/MMBR.00055-16 (2017).

19. Stiasny, K., Kiermayr, S., Holzmann, H. \& Heinz, F. X. Cryptic properties of a cluster of dominant flavivirus cross-reactive antigenic sites. Journal of virology 80, 9557-9568, https://doi.org/10.1128/JVI.00080-06 (2006).

20. Kraemer, M. U. et al. The global distribution of the arbovirus vectors Aedes aegypti and Ae. albopictus. Elife 4, e08347, https://doi. org/10.7554/eLife.08347 (2015)

21. Balmaseda, A. et al. Comparison of four serological methods and two RT-PCR assays for diagnosis and surveillance of Zika. J Clin Microbiol, https://doi.org/10.1128/JCM.01785-17 (2018).

22. Premkumar, L. et al. Development of Envelope Protein Antigens To Serologically Differentiate Zika Virus Infection from Dengue Virus Infection. J Clin Microbiol 56, https://doi.org/10.1128/JCM.01504-17 (2018).

23. Tsai, W. Y. et al. Distinguishing Secondary Dengue Virus Infection From Zika Virus Infection With Previous Dengue by a Combination of 3 Simple Serological Tests. Clin Infect Dis 65, 1829-1836, https://doi.org/10.1093/cid/cix672 (2017).

24. Balmaseda, A. et al. Antibody-based assay discriminates Zika virus infection from other flaviviruses. Proc. Natl. Acad. Sci. USA 114, 8384-8389, https://doi.org/10.1073/pnas.1704984114 (2017).

25. Hotop, S. K. et al. Multiple antibody targets on herpes B glycoproteins B and D identified by screening sera of infected rhesus macaques with peptide microarrays. Plos one 9, e86857, https://doi.org/10.1371/journal.pone.0086857 (2014).

26. Kostyuchenko, V. A. et al. Structure of the thermally stable Zika virus. Nature 533, 425-428, https://doi.org/10.1038/nature17994 (2016).

27. Homan, E. J., Malone, R. W., Darnell, S. J. \& Bremel, R. D. Antibody mediated epitope mimicry in the pathogenesis of Zika virus related disease. bioRxiv, https://doi.org/10.1101/044834 (2016).

28. Xu, X. et al. Identifying Candidate Targets of Immune Responses in Zika Virus Based on Homology to Epitopes in Other Flavivirus Species. PLoS currents 8, https://doi.org/10.1371/currents.outbreaks.9aa2e1fb61b0f632f58a098773008c4b (2016).

29. Kaida, K., Ariga, T. \& Yu, R. K. Antiganglioside antibodies and their pathophysiological effects on Guillain-Barre syndrome and related disorders-a review. Glycobiology 19, 676-692, https://doi.org/10.1093/glycob/cwp027 (2009).

30. Zammarchi, L. et al. Zika virus infection in a traveller returning to Europe from Brazil, March 2015. Euro surveillance: bulletin Europeen sur les maladies transmissibles = European communicable disease bulletin 20 (2015).

31. Frank, R. Spot-Synthesis - an Easy Technique for the Positionally Addressable, Parallel Chemical Synthesis on a Membrane Support. Tetrahedron 48, 9217-9232, https://doi.org/10.1016/S0040-4020(01)85612-X (1992).

32. Dikmans, A., Beutling, U., Schmeisser, E., Thiele, S. \& Frank, R. SC2: A novel process for manufacturing multipurpose high-density chemical microarrays. Qsar \& Combinatorial Science 25, 1069-1080, https://doi.org/10.1002/Qsar.200640130 (2006).

\section{Acknowledgements}

The authors thank Gabriela Salinas and Susanne Luthin, DNA Microarray Facility, University Medical Centre Goettingen, Germany, for giving us the chance to use the Agilent microarray scanner and for helpful technical support.

\section{Author Contributions}

S.H., S.K.H., F.H., M.B., H.J.F. and A.A.E.W. designed the study. S.H., S.K.H., O.F., O.N., S.B.F., R.P., F.H., C.S.H., R.F., C.P.C., J.S.C., S.S.S., A.A.S., M.N., M.B., H.J.F. and A.A.E.W. collected the samples and/or did laboratory work. S.H., S.K.H., F.H., M.B. and A.A.E.W. analysed and interpreted the data. S.H., H.J.F. and A.A.E.W. wrote the manuscript. The manuscript was critically reviewed, edited, and approved by all authors.

Additional Information

Supplementary information accompanies this paper at https://doi.org/10.1038/s41598-019-40224-2. 
Competing Interests: The authors declare no competing interests.

Publisher's note: Springer Nature remains neutral with regard to jurisdictional claims in published maps and institutional affiliations.

(c) (i) Open Access This article is licensed under a Creative Commons Attribution 4.0 International License, which permits use, sharing, adaptation, distribution and reproduction in any medium or format, as long as you give appropriate credit to the original author(s) and the source, provide a link to the Creative Commons license, and indicate if changes were made. The images or other third party material in this article are included in the article's Creative Commons license, unless indicated otherwise in a credit line to the material. If material is not included in the article's Creative Commons license and your intended use is not permitted by statutory regulation or exceeds the permitted use, you will need to obtain permission directly from the copyright holder. To view a copy of this license, visit http://creativecommons.org/licenses/by/4.0/.

(C) The Author(s) 2019 


\section{Chapter VI: General Discussion}

In general, the work of this PhD thesis consists of three parts. In the first part (Chapter II), a field applicable method was developed in order to extract nucleic acids from complex samples. The second part (Chapter III and IV) of the PhD study focused on the genomic identification of pathogens by applying diagnostic metagenomics in the field. The third part of this thesis was dedicated to the identification of specific serological markers, especially those useful for the species differentiation among the genus of flaviviruses.

Why Mycobacterium avium subspecies paratuberculosis, Zika virus and Foot and Mouth disease virus were selected as model pathogens in this PhD work

MAP, causing paratuberculosis or John's disease, is a Gam-positive, non-motile, nonspore forming and acid-fast bacterium. It infects mainly ruminants leading to diarrhoea and losses in weight and milk production [103, 104]. The number of infected and shedding animals within a herd is hard to estimate, as the bacteria is expelled from the body in intervals and clinical signs of the paratuberculosis appeared only in a small number of infected animals [63, 105]. Faecal-oral route is seen as the main way of transmission [106]. Therefore, the identification of the bacterium in faeces is very important. However, culturing of the bacteria, which is seen as the gold standard of diagnostics, needs at least 12 weeks to result [107]. As stated in Chapter II, nucleic acid amplification assays are available for the detection of MAP, but the sensitivity of these methods depends highly on the performance of the nucleic acid extraction. The extraction of DNA from MAP in faecal samples is very challenging. The bacteria are resistant to many chemical and physical forces due to the thick, lipid and polysaccharose rich cell wall. In addition, the diverse compounds in the faeces may inhibit the reaction [108-110]. Thus, MAP is an optimal candidate for the development of a protocol for nucleic acid extraction, which is also suitable for other challenging pathogens and sample matrixes.

FMDV is a highly contagious virus from the family of Picornaviridae. The virus causes high economical losses by infecting mainly cloven-hoofed animals. The European Union is free from the virus, but there is a risk of re-emergence, as the virus is endemic in Northern Africa and the Middle East [111]. A critical point in transmission is the viral genome, which can directly act as messenger RNA within the host without the need of an intact virion [112]. The seven serotypes of FMDV have no cross-reactivity between 
each other. Accordingly, vaccination provides no cross-protection. In addition, there is a strong genetic variability within each serotype [113]. ELISA is the common laboratory method for serotype identification [114] but there is a lack of approaches for differentiation on genomic base and at PON. All molecular assays like RPA [115] and real-time PCR [116] detect only the virus genome but cannot be used for FMDV differentiation. Attempts to apply such assays for detection of the FMDV serotypes were only restricted to the strains circulating in a particular area $[117,118]$. Therefore, FMDV is a good example for applying sequencing as a universal method for the discrimination between closely related viral strains.

ZIKV (Figure 6) is a prominent representative of the genus of flaviviruses [119]. More than 50 arthropod borne species are located in this genus (Figure 7, [120]) infecting not only human but also wild and domestic animals. Flaviviruses are listed in both the OIE list of highly infectious diseases for animals as well as the neglected viral diseases by the WHO $[100,121,122]$. Besides ZIKV, the genus of flaviviruses includes pathogens like Dengue, Yellow Fever virus, West Nile and Japanese Encephalitis virus [123].

ZIKV was declared temporarily as a public health emergency by the World Health Organization in 2016 due to the South American outbreak at that time [124]. Although $\mathrm{ZIKV}$ is no longer seen as a public health emergency, the virus is still listed as priority disease [125] .

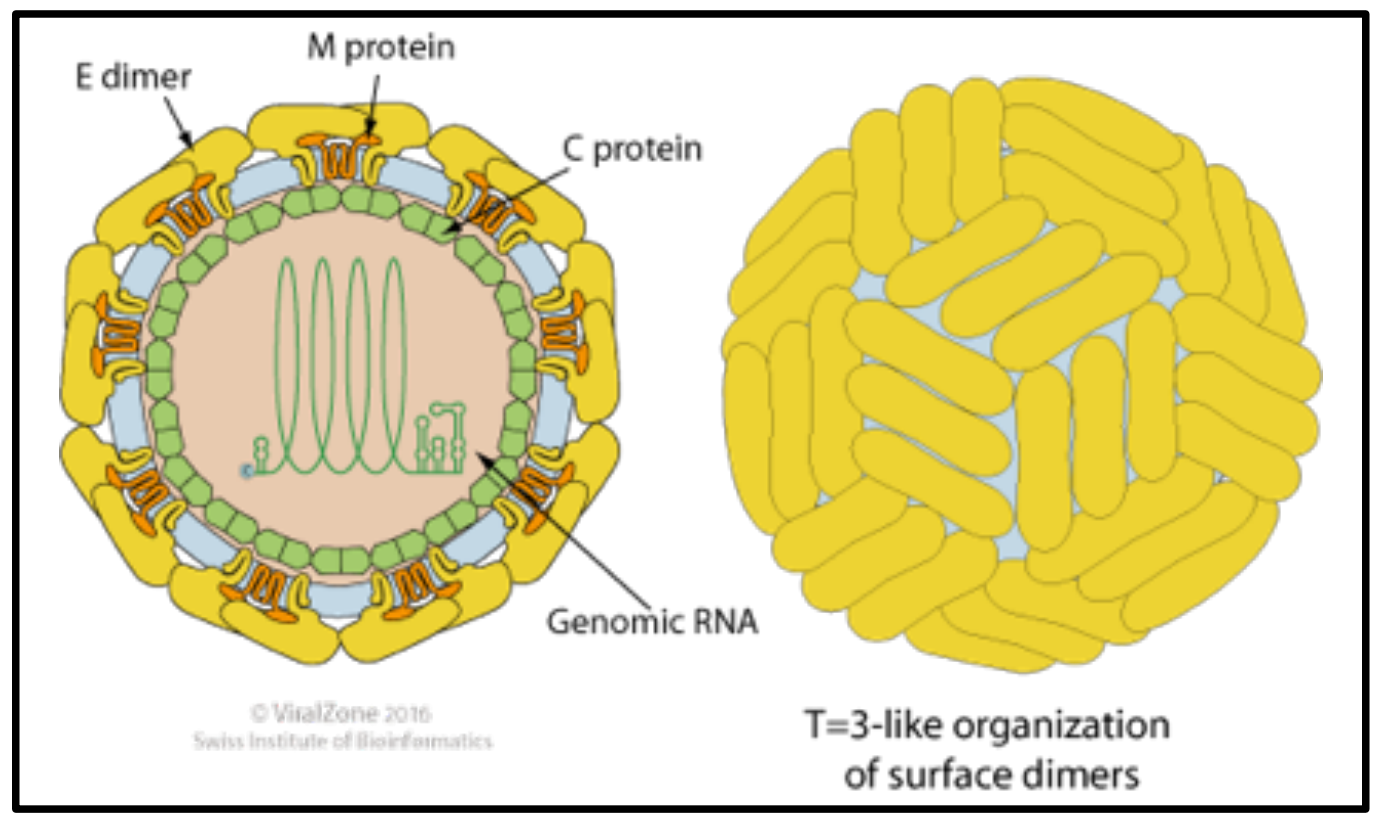

Figure 6: Schematic picture of ZIKV (Panel A) Source: ViralZone [126].

The reservoir of ZIKV is not clearly identified, although non-human primates are shown to be susceptible to the virus [127]. Natural infection with the virus was recorded in 
other domestic animals, but was only seen on antibody basis [128], which is not a clear proof due to the antigenic homology to other flaviviruses. According to the United States Centers for Disease Control and Prevention (CDC), there are no reports of ZIKV infected animal despite a single study, which showed that identified ZIKV positive animals have no clinical signs or the potential to spread the virus [129].

ZIKV and other flaviviruses like the Dengue and West Nile virus often circulate in the same regions [130-132] and have similar clinical signs like unspecific fibril illness [133], but clinical manifestations within the genus can differ from haemorrhagic fever to vasculopathy or encephalitis [134]. The viremia lasts only for around five days with a very low virus concentration in the blood ( 3 viral particles per $\mathrm{ml}$ blood, $[135,136]$ ). However, most sequencing methods require around $10^{4}$ molecules per tested sample to generate a nucleic acid sequence [137]. Therefore, ZIKV was considered a suitable virus to proof the concept of combining random isothermal amplification and nanopore sequencing in order to achieve the required amount of DNA needed (Chapter III).

Flaviviruses have a high antigenic homology to each other and cross-reactivity in immunological tests is quite common (Figure $7,[123,138]$ ). Hence, flaviviruses can be sorted into groups of two or more viruses, which are distinct from each other, however, related serologically. These groups are called serogroups. Within the serogroup, viruses more antigenically close to each other are forming a serocomplex [139, 140]). ZIKV is in the same serogroup of dengue virus and ZIKV and Spondweni virus are forming a serocomplex (Figure 7). A discrimination between acute ZIKV infection and a previous Dengue infection is difficult because both are located under the same serogroup [136].

Thus, identification of ZIKV specific serological markers in order to identify an ZIKV infection against the background of dengue virus and other ongoing or previous flavivirus infections is very urgent to guarantee the right case management. 


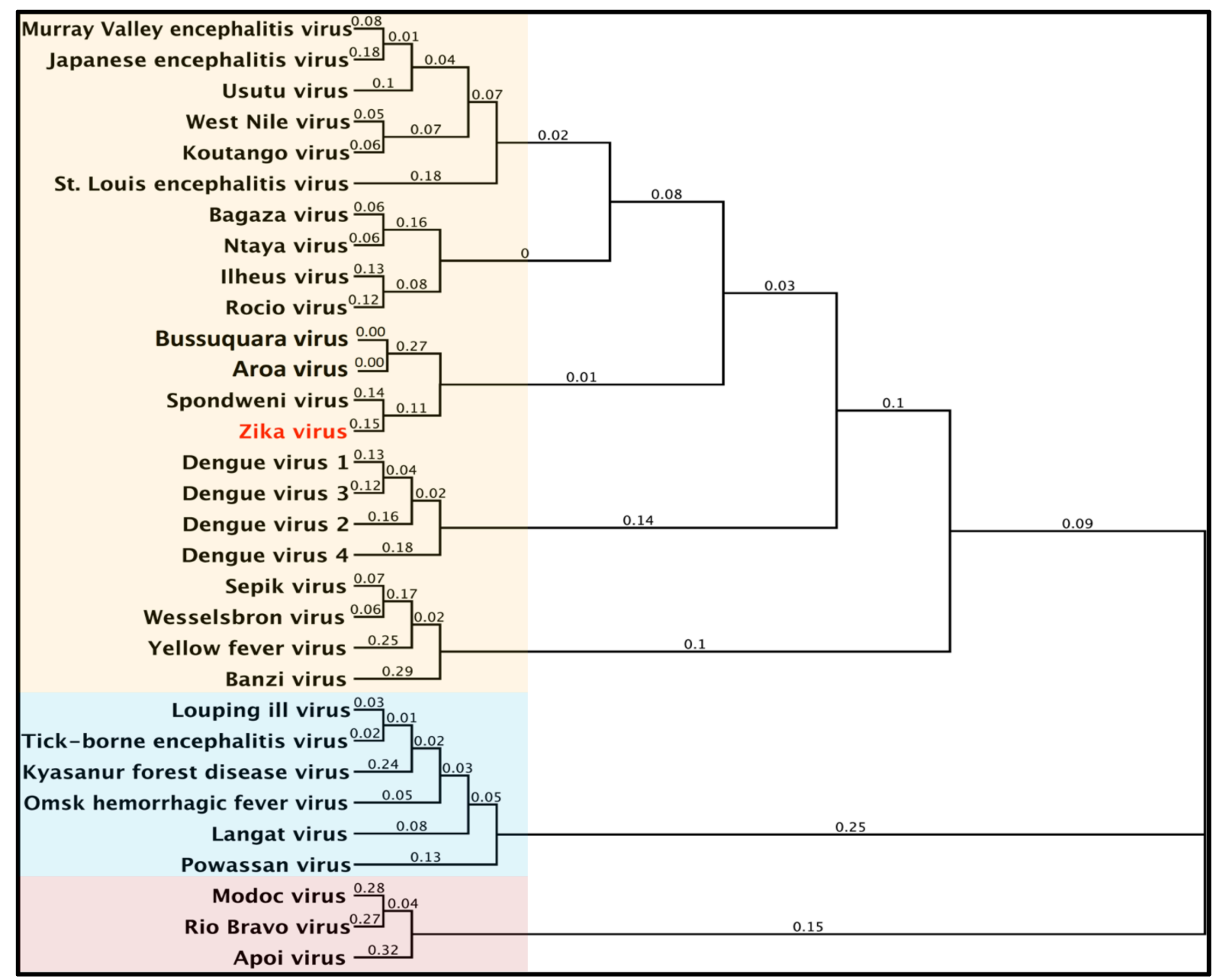

Figure 7: Phylogenetic relationship of selected flaviviruses based on the divergence of the full amino acid sequences. The branch labels show the difference between the single serogroups and species, coloured background displays which viruses form a serogroup.

\section{The extraction of bacterial DNA in the field}

The rapid isolation of nucleic acids in the field is urgent for the diagnostics on a genomic or metagenomic base at POC and PON. In this $\mathrm{PhD}$ work, a rapid and sensitive protocol for the extraction of bacteria was developed (Chapter II). The major advantage of this protocol is the bead beating step in the field, which facilitates the isolation of DNA from Gram-positive bacteria with a strong cell wall [141].

The SpeedXtract method (Qiagen Hilden, Hilden, Germany) is a rapid nucleic acid extraction method which can easily be performed in the field requiring a heating source capable of $95{ }^{\circ} \mathrm{C}$ and a magnetic stand. The protocol includes only a few pipetting steps and can be taught to untrained persons in less than one day.

The technology relies on the reverse purification of the sample, i. e. after cell lysis, the inhibitors for nucleic acid amplification like cell membranes are removed from the supernatant, but other molecules like DNA and RNA in addition to coloured particles 
(bile salts, [142]) remain. Therefore, the technology is only compatible with amplification methods resistant to inhibitors like RPA, but not with PCR.

Although the SpeedXtract method was used for the nucleic acid extraction from parasites and viruses before [68, 143-145], the protocol developed in this $\mathrm{PhD}$ thesis is the first, using the reverse purification technology for bacteria.

\section{Sequencing protocols for the rapid identification of the causative agent at}

\section{outbreak situations}

Metagenomic diagnostics are a reliable and necessary tool for the identification of pathogens responsible in an outbreak situation. In this $\mathrm{PhD}$ work, two protocols were developed for the use of the nanopore sequencing in the field. The first protocol is dedicated to the sequencing of ZIKV (Chapter III). As the viral load of ZIKV within a sample is only around 3 particles per millilitre of blood [135], a random isothermal amplification step was implemented after reverse transcription and second strand synthesis in order to increase the total yield of the of DNA.

Sequencing of RNA viruses at PON was done before employing nanopore sequencing. Quick et al. performed reverse transcription PCR (RT-PCR) using 38 pairs of Ebola virus specific primers targeting the whole genome before sequencing. This combination of targeted RT-PCR and nanopore sequencing was applied to reduce the human background within the sequences. The produced sequences covered around 97 percent of the complete viral genome [57]. As the specific primers were selected from a known reference sequence, variants in the targeted genome sequence might have led to a decrease in priming efficacy. Moreover, large rearrangements like insertions or deletions are only detected, when flanked by primers [146]. These might be the reason for the incomplete sequencing of the full Ebola genome. In contrast, the random hexamer primers and Multiple Displacement Amplification technology applied in this $\mathrm{PhD}$ work hybridize to the cDNA distributed over the whole genome sequence and produce amplicons between 2-100 kb [147]. Therefore, the random isothermal amplification in combination with nanopore sequencing is superior by yielding long sequences and full coverage of the sequenced genome without the need of heavy and complex devices such as a thermocycler.

The second sequencing protocol was developed for the rapid serotyping of FMDV in the field via metagenomic diagnostics (Chapter IV). The FMDV genome consists of a single stranded positive sense RNA (ss-RNA) with a poly-A tail at the $3^{`}$ end [148]. In 
order to create a full-length DNA, a poly-T reverse primer was applied together with the SMART-technology (Zhu et al., 2001, [149]). As consequence, a non-fragmented long DNA molecule was produced. Sequence analysis using long reads has the advantage of being more accurate and deliver results similar to the natural situation [150].

The major breakthrough approach of the two metagenomic diagnostics studies is the combination of direct sequencing with the local offline BLAST search. The implementation of an offline BLAST database in outbreak situations liberates from the need of a stable internet connection especially under field settings. The GENEIOUS v9.1.6 (Biomatters Ltd., Auckland, New Zealand) was employed for the offline database and sequence analysis. As the program is very user friendly, a bioinformatic education is not needed.

Metagenomic diagnostics in the field is only possible with portable sequencing devices. Hitherto, the MinION device is the only portable sequencer suitable for the use in the field. Nevertheless, an expensive high-performance computer was required for realtime sequence data collection and analysis. To improve the field applicability, mobile data acquisition hardware was developed (MinION (Figure 5), MinIT, or Mk1C by ONT). All these developments allowed sequencing without the drawbacks of using incompatible laptop. Another issue, the quality of the sequencing data depends on the amount and condition of the DNA [151]. Therefore, the DNA integrity must be checked after each step of the library preparation to assure an excellent sequencing run.

\section{Differentiation of Zika virus to other flaviviruses using peptide microarrays}

Most serological tests for the identification of flaviviruses detect antibodies directed against the envelope $(E)$ protein or non-structural 1 (NS1) protein [152]. The E protein consists out of three parts (DI-DIII) which are organized as antiparallel homodimers. Antibodies can attach easily to the outer parts of the virus and be directed to more than one part of the enveloped (inter dimer epitope, [153]) The NS1 protein is highly conserved among all flaviviruses and takes part in immune evasion and genetic replication of the virus [154]. Virus specific epitopes to other non-structural proteins were also identified [155, 156], but not deeply characterized. In this PhD work, a peptide microarray was applied to identify 68 ATRs, from which 13 were considered as ZIKV specific. These ATRs are potential candidates for the development of simpler serological test for ZIKV infection. 
Several attempts were made to differentiate ZIKV to other flaviviruses on a serological basis. Premkumar et al. propose two specific epitopes by prediction followed by producing and testing the respective synthetic antigens in ELISA [157]. The study focused only on the envelope proteins and did not consider the other immunogenic proteins. In two studies, ELISA assays were developed $[158,159]$ but each of these assays only utilized a single ZIKV epitope. All three studies concentrated on the differentiation of ZIKV to Dengue virus and did not consider other flaviviruses. Other authors employed peptide microarray chips to identify epitopes for discrimination of ZIKV to other flaviviruses [160, 161]. The first study relies on macaque sera [160], while the second study targeted a sole antigenic site on NS2b [161]. The epitope mentioned in these studies was partly mapped in this PhD work (Chapter V; epitope 1441). Surprisingly, the epitope was not seen as ZIKV specific but recognized only by FlaviMix pool sera instead.

Mapping targets of antibodies against ZIKV is crucial, particularly to elucidate the cross reactivity to other flaviviruses $[123,162,163]$ in order to provide accurate identification of infected cases. Several methods (e.g. phage display libraries and peptide-based ELISA) have been used [164]. These, however, suffer from cumbersome handling and the need for large amounts of sera. The use of peptide microarrays has the advantage of identifying simultaneous antibody binding sites over the whole polyprotein in highresolution at individual epitope level [165]. Thousands of target peptides can be analysed in one run by using only a small volume of diluted sample with a high reproducibility [166]. The technique can even identify shifts in epitopes on the same protein [167].

A big drawback of the peptide microarrays is that only linear epitopes can be identified, conformational epitopes or discontinuous epitopes can only be partly mapped [168]. In order to map other epitope types, alternative methods should be applied [169]. The peptides printed onto the microarray glass slide are produced in relatively large volume which then can be stored in a deep freezer for later use. Due to this kind of storage, a variation in performance between the different batches of microarray slides was recorded [170]. In this $\mathrm{PhD}$ work, studies using peptide microarrays produce a huge amount of data. Read out and interpretation of the data can be achieved by special bioinformatics tools [171-173]. Nevertheless, the presence of a strong background 
interferes with the automated positive signal recording. A manual data acquisition was necessary to count the true positive signals, but it was time consuming.

The complexity of ZIKV clinical picture and possible multiple transmission routes as well as high antibody cross-reactivity between flaviviruses make it difficult to identify the ZIKA clinical cases and decode the ZIKV pathogenesis. There is a need to apply novel technologies in order to reveal the glaring discrepancy between the complex clinical picture of ZIKV and the lack of scientific evidence bearing explanatory potential. The ZIKV peptide microarray allowed high-resolution mapping of the ZIKV antibody linear target, which can be used to develop a POCT. Nevertheless, additional investigations are essential to determine the biological function of these antibodies.

\section{Conclusion}

In the last three decades, more than 30 new infectious diseases emerged, most of them zoonotic [174]. To prevent them from crossing species barriers or spread globally, a reliable method for the identification of pathogens is needed urgently.

Current POCT and PONT are often designed for the detection of a single pathogen and do not have the diagnostic capabilities of laboratory tests. A platform suitable for the investigation of multiple pathogens is needed in order to identify the causative pathogen of an outbreak. Once the pathogen is detected, a target-specific rapid test kit must be deployed to recognize the diseased individuals.

This PhD work presented several approaches for better development of diagnostics tests at PON and POC. On one hand, the fast and accurate extraction of DNA enabled the use of molecular assays at PON. On the other hand, the adaption of the two sequencing protocols to the mobile suitcase laboratory plus the offline data analysis provided metagenomic diagnostics under field conditions. The use of peptide microarray technology for identification of a bundle of potential ZIKV specific immunologic markers was demonstrated. This will lead to better specific serological test for the detection of closely related viruses. 


\section{Chapter VII: References}

1. World Health Organization. Infectious diseases 2019 [24.04.2019]. Available from: https://www.who.int/topics/infectious diseases/en/.

2. Fonkwo PN. Pricing infectious disease. The economic and health implications of infectious diseases. EMBO Rep. 2008;9 Suppl 1:S13-7. doi: 10.1038/embor.2008.110. PubMed PMID: 18578017; PubMed Central PMCID: PMCPMC3327542.

3. World Health Organization. Economic burden of disease 2009 [24.04.2019]. Available from: https://www.who.int/choice/publications/d_economic_impact_guide.pdf?ua=1.

4. World Organization for Animal Health. Feeding the world better by controlling animal diseases 2019 [27.04.2019]. Available from: http://www.oie.int/for-the-media/editorials/detail/article/feeding-the-world-better-by-controlling-animal-diseases/.

5. Magnusson R. Chapter 10: Controlling the spread of infectious diseases. Advancing the right to health: the vital role of law2017.

6. Schito M, Peter TF, Cavanaugh S, Piatek AS, Young GJ, Alexander H, et al. Opportunities and challenges for cost-efficient implementation of new point-of-care diagnostics for HIV and tuberculosis. J Infect Dis. 2012;205 Suppl 2:S169-80. doi: 10.1093/infdis/jis044. PubMed PMID: 22457286; PubMed Central PMCID: PMCPMC3334507.

7. Peeling RW, Holmes KK, Mabey D, Ronald A. Rapid tests for sexually transmitted infections (STIs): the way forward. Sex Transm Infect. 2006;82 Suppl 5:v1-6. doi: 10.1136/sti.2006.024265. PubMed PMID: 17151023; PubMed Central PMCID: PMCPMC2563912.

8. Hanon JB, Vandenberge V, Deruelle M, De Leeuw I, De Clercq K, Van Borm S, et al. Inter-laboratory evaluation of the performance parameters of a Lateral Flow Test device for the detection of Bluetongue virus-specific antibodies. J Virol Methods. 2016;228:140-50. doi: 10.1016/j.jviromet.2015.12.001. PubMed PMID: 26687976.

9. Aebischer A, Wernike K, Hoffmann B, Beer M. Rapid genome detection of Schmallenberg virus and bovine viral diarrhea virus by use of isothermal amplification methods and high-speed real-time reverse transcriptase PCR. J Clin Microbiol. 2014;52(6):1883-92. doi: 10.1128/JCM.00167-14. PubMed PMID: 24648561; PubMed Central PMCID: PMCPMC4042763.

10. Jain B, Lambe U, Tewari A, Kadian SK, Prasad M. Development of a rapid test for detection of foot-and-mouth disease virus specific antibodies using gold nanoparticles. Virusdisease. 2018:29(2):192-8. doi: 10.1007/s13337-018-0450-8. PubMed PMID: 29911152; PubMed Central PMCID: PMCPMC6003053.

11. Shalaby MA, El-Deeb A, El-Tholoth M, Hoffmann D, Czerny CP, Hufert FT, et al. Recombinase polymerase amplification assay for rapid detection of lumpy skin disease virus. BMC Vet Res. 2016;12(1):244. doi: 10.1186/s12917-016-0875-5. PubMed PMID: 27806722; PubMed Central PMCID: PMCPMC5094145.

12. Derda R, Gitaka J, Klapperich CM, Mace CR, Kumar AA, Lieberman M, et al. Enabling the Development and Deployment of Next Generation Point-of-Care Diagnostics. PLoS Negl Trop Dis. 2015;9(5):e0003676. doi: 10.1371/journal.pntd.0003676. PubMed PMID: 25973602; PubMed Central PMCID: PMCPMC4431858.

13. Thiam S, Thior M, Faye B, Ndiop M, Diouf ML, Diouf MB, et al. Major reduction in anti-malarial drug consumption in Senegal after nation-wide introduction of malaria rapid diagnostic tests. PLoS One. 2011;6(4):e18419. doi: 10.1371/journal.pone.0018419. PubMed PMID: 21494674; PubMed Central PMCID: PMCPMC3071817.

14. Kozel TR, Burnham-Marusich AR. Point-of-Care Testing for Infectious Diseases: Past, Present and Future. J Clin Microbiol. 2017;55(8):2313-20. doi: 10.1128/JCM.00476-17. PubMed PMID: 28539345; PubMed Central PMCID: PMCPMC5527409.

15. Sakamoto S, Putalun W, Vimolmangkang S, Phoolcharoen W, Shoyama $Y$, Tanaka H, et al. Enzyme-linked immunosorbent assay for the quantitative/qualitative analysis of plant secondary metabolites. J Nat Med. 2018;72(1):32-42. doi: 10.1007/s11418-017-1144-z. PubMed PMID: 29164507; PubMed Central PMCID: PMCPMC5775980.

16. Belanger L, Sylvestre C, Dufour D. Enzyme-linked immunoassay for alpha-fetoprotein by competitive and sandwich procedures. Clin Chim Acta. 1973;48(1):15-8. PubMed PMID: 4201034

17. Sajid M, Kawde A-N, Daud M. Designs, formats and applications of lateral flow assay: A literature review. Journal of Saudi Chemical Society. 2015;19(6):689-705. doi: https://doi.org/10.1016/j.jscs.2014.09.001.

18. van Amerongen A, Veen J, Arends HA, Koets M. Chapter 7 - Lateral Flow Immunoassays. In: Vashist SK, Luong JHT, editors. Handbook of Immunoassay Technologies: Academic Press; 2018. p. 157-82.

19. Koczula KM, Gallotta A. Lateral flow assays. Essays Biochem. 2016:60(1):111-20. doi: 10.1042/EBC20150012. PubMed PMID: 27365041; PubMed Central PMCID: PMCPMC4986465

20. Anfossi L, Di Nardo F, Cavalera S, Giovannoli C, Baggiani C. Multiplex Lateral Flow Immunoassay: An Overview of Strategies towards High-throughput Point-of-Need Testing. Biosensors (Basel). 2018;9(1). doi: 10.3390/bios9010002. PubMed PMID: 30587769

21. He ZX, Shi LC, Ran XY, Li W, Wang XL, Wang FK. Development of a Lateral Flow Immunoassay for the Rapid Diagnosis of Invasive Candidiasis. Front Microbiol. 2016;7:1451. doi: 10.3389/fmicb.2016.01451. PubMed PMID: 27679622; PubMed Central PMCID: PMCPMC5020066.

22. Lee S, Mehta S, Erickson D. Two-Color Lateral Flow Assay for Multiplex Detection of Causative Agents Behind Acute Febrile Illnesses. Anal Chem. 2016;88(17):8359-63. doi: 10.1021/acs.analchem.6b01828. PubMed PMID: 27490379; PubMed Central PMCID: PMCPMC5396465.

23. Boutal $\mathrm{H}$, Vogel A, Bernabeu S, Devilliers $\mathrm{K}$, Creton $\mathrm{E}$, Cotellon $\mathrm{G}$, et al. A multiplex lateral flow immunoassay for the rapid identification of NDM-, KPC-, IMP- and VIM-type and OXA-48-like carbapenemase-producing Enterobacteriaceae. J Antimicrob Chemother. 2018;73(4):909-15. doi: 10.1093/jac/dkx521. PubMed PMID: 29365094; PubMed Central PMCID: PMCPMC5890661. 
24. Tenda K, van Gerven B, Arts R, Hiruta Y, Merkx M, Citterio D. Paper-Based Antibody Detection Devices Using Bioluminescent BRET-Switching Sensor Proteins. Angew Chem Int Ed Engl. 2018;57(47):15369-73. doi: 10.1002/anie.201808070. PubMed PMID: 30168634; PubMed Central PMCID: PMCPMC6282528.

25. Yang Y, Noviana E, Nguyen MP, Geiss BJ, Dandy DS, Henry CS. Paper-Based Microfluidic Devices: Emerging Themes and Applications. Anal Chem. 2017;89(1):71-91. doi: 10.1021/acs.analchem.6b04581. PubMed PMID: 27936612.

26. Sher M, Zhuang R, Demirci U, Asghar W. Paper-based analytical devices for clinical diagnosis: recent advances in the fabrication techniques and sensing mechanisms. Expert Rev Mol Diagn. 2017;17(4):351-66. doi: 10.1080/14737159.2017.1285228. PubMed PMID: 28103450; PubMed Central PMCID: PMCPMC5529145.

27. Mu X, Zhang L, Chang S, Cui W, Zheng Z. Multiplex microfluidic paper-based immunoassay for the diagnosis of hepatitis $\mathrm{C}$ virus infection. Anal Chem. 2014;86(11):5338-44. doi: 10.1021/ac500247f. PubMed PMID: 24794788.

28. Cho DG, Yoo H, Lee H, Choi YK, Lee M, Ahn DJ, et al. High-Speed Lateral Flow Strategy for a Fast Biosensing with an Improved Selectivity and Binding Affinity. Sensors (Basel). 2018;18(5). doi: 10.3390/s18051507. PubMed PMID: 29748509; PubMed Central PMCID: PMCPMC5982462.

29. Posthuma-Trumpie GA, Korf J, van Amerongen A. Lateral flow (immuno)assay: its strengths, weaknesses, opportunities and threats. A literature survey. Anal Bioanal Chem. 2009;393(2):569-82. doi: 10.1007/s00216-008-2287-2. PubMed PMID: 18696055.

30. Kary B. Mullis, Henry A. Erlich, Norman Arnheim, Glenn T. Horn, Randall K. Saiki, Scharf SJ, inventorsProcess for amplifying, detecting, and/or cloning nucleic acid sequences. United States of America1986.

31. Mackay IM, Arden KE, Nitsche A. Real-time PCR in virology. Nucleic Acids Res. 2002;30(6):1292-305. PubMed PMID: 11884626; PubMed Central PMCID: PMCPMC101343.

32. Murray JL, Hu P, Shafer DA. Seven novel probe systems for real-time PCR provide absolute single-base discrimination, higher signaling, and generic components. J Mol Diagn. 2014;16(6):627-38. doi: 10.1016/j.jmoldx.2014.06.008. PubMed PMID: 25307756; PubMed Central PMCID: PMCPMC4210465.

33. Zanoli LM, Spoto G. Isothermal amplification methods for the detection of nucleic acids in microfluidic devices. Biosensors (Basel). 2013;3(1):18-43. doi: 10.3390/bios3010018. PubMed PMID: 25587397; PubMed Central PMCID: PMCPMC4263587.

34. Boyle DS, McNerney R, Teng Low H, Leader BT, Perez-Osorio AC, Meyer JC, et al. Rapid detection of Mycobacterium tuberculosis by recombinase polymerase amplification. PLoS One. 2014;9(8):e103091. doi: 10.1371/journal.pone.0103091. PubMed PMID: 25118698; PubMed Central PMCID: PMCPMC4138011.

35. Craw $P$, Balachandran $W$. Isothermal nucleic acid amplification technologies for point-of-care diagnostics: a critical review. Lab Chip. 2012;12(14):2469-86. doi: 10.1039/c2lc40100b. PubMed PMID: 22592150.

36. Notomi T, Okayama H, Masubuchi H, Yonekawa T, Watanabe K, Amino N, et al. Loop-mediated isothermal amplification of DNA. Nucleic Acids Res. 2000;28(12):E63. PubMed PMID: 10871386; PubMed Central PMCID: PMCPMC102748.

37. Piepenburg $\mathrm{O}$, Williams $\mathrm{CH}$, Stemple $\mathrm{DL}$, Armes NA. DNA detection using recombination proteins. PLoS Biol. 2006;4(7):e204. doi: 10.1371/journal.pbio.0040204. PubMed PMID: 16756388; PubMed Central PMCID: PMCPMC1475771.

38. Ye X, Xu J, Lu L, Li X, Fang X, Kong J. Equipment-free nucleic acid extraction and amplification on a simple paper disc for point-of-care diagnosis of rotavirus A. Anal Chim Acta. 2018;1018:78-85. doi: 10.1016/j.aca.2018.02.068. PubMed PMID: 29605138.

39. Seok Y, Joung HA, Byun JY, Jeon HS, Shin SJ, Kim S, et al. A Paper-Based Device for Performing Loop-Mediated Isothermal Amplification with Real-Time Simultaneous Detection of Multiple DNA Targets. Theranostics. 2017;7(8):222030. doi: 10.7150/thno.18675. PubMed PMID: 28740546; PubMed Central PMCID: PMCPMC5505055.

40. Linnes JC, Fan A, Rodriguez NM, Lemieux B, Kong H, Klapperich CM. Paper-based molecular diagnostic for Chlamydia trachomatis. RSC Adv. 2014;4(80):42245-51. doi: 10.1039/C4RA07911F. PubMed PMID: 25309740; PubMed Central PMCID: PMCPMC4188396.

41. LaBarre P, Boyle D, Hawkins K, Weigl B. Instrument-free nucleic acid amplification assays for global health settings. Proc SPIE Int Soc Opt Eng. 2011;8029. doi: 10.1117/12.882868. PubMed PMID: 25089171; PubMed Central PMCID: PMCPMC4116599.

42. LaBarre P, Hawkins KR, Gerlach J, Wilmoth J, Beddoe A, Singleton J, et al. A simple, inexpensive device for nucleic acid amplification without electricity-toward instrument-free molecular diagnostics in low-resource settings. PLoS One. 2011;6(5):e19738. doi: 10.1371/journal.pone.0019738. PubMed PMID: 21573065; PubMed Central PMCID: PMCPMC3090398.

43. Gadkar VJ, Goldfarb DM, Gantt S, Tilley PAG. Real-time Detection and Monitoring of Loop Mediated Amplification (LAMP) Reaction Using Self-quenching and De-quenching Fluorogenic Probes. Sci Rep. 2018;8(1):5548. doi: 10.1038/s41598-01823930-1. PubMed PMID: 29615801; PubMed Central PMCID: PMCPMC5883045.

44. Zasada AA, Zacharczuk K, Forminska K, Wiatrzyk A, Ziolkowski R, Malinowska E. Isothermal DNA amplification combined with lateral flow dipsticks for detection of biothreat agents. Anal Biochem. 2018;560:60-6. doi: 10.1016/j.ab.2018.09.008. PubMed PMID: 30217500.

45. Liu M, Hui CY, Zhang Q, Gu J, Kannan B, Jahanshahi-Anbuhi S, et al. Target-Induced and Equipment-Free DNA Amplification with a Simple Paper Device. Angew Chem Int Ed Engl. 2016;55(8):2709-13. doi: 10.1002/anie.201509389. PubMed PMID: 26748431. 
46. Liu L, Wang J, Zhang R, Lin M, Shi R, Han Q, et al. Visual and equipment-free reverse transcription recombinase polymerase amplification method for rapid detection of foot-and-mouth disease virus. BMC Vet Res. 2018;14(1):263. doi: 10.1186/s12917-018-1594-x. PubMed PMID: 30170587; PubMed Central PMCID: PMCPMC6119248.

47. Crannell ZA, Rohrman B, Richards-Kortum R. Equipment-free incubation of recombinase polymerase amplification reactions using body heat. PLoS One. 2014;9(11):e112146. doi: 10.1371/journal.pone.0112146. PubMed PMID: 25372030; PubMed Central PMCID: PMCPMC4221156.

48. Liu L, Wang J, Geng Y, Wang J, Li R, Shi R, et al. Equipment-free recombinase polymerase amplification assay using body heat for visual and rapid point-of-need detection of canine parvovirus 2. Mol Cell Probes. 2018;39:41-6. doi: 10.1016/j.mcp.2018.04.004. PubMed PMID: 29705183.

49. Kersting S, Rausch V, Bier FF, von Nickisch-Rosenegk M. Rapid detection of Plasmodium falciparum with isothermal recombinase polymerase amplification and lateral flow analysis. Malar J. 2014;13:99. doi: 10.1186/1475-2875-13-99. PubMed PMID: 24629133; PubMed Central PMCID: PMCPMC4004163.

50. Pallen MJ. Diagnostic metagenomics: potential applications to bacterial, viral and parasitic infections. Parasitology. 2014;141(14):1856-62. doi: 10.1017/S0031182014000134. PubMed PMID: 24576467; PubMed Central PMCID: PMCPMC4255322.

51. Forbes JD, Knox NC, Peterson CL, Reimer AR. Highlighting Clinical Metagenomics for Enhanced Diagnostic Decisionmaking: A Step Towards Wider Implementation. Comput Struct Biotechnol J. 2018;16:108-20. doi: 10.1016/j.csbj.2018.02.006. PubMed PMID: 30026887; PubMed Central PMCID: PMCPMC6050174.

52. Greninger AL. The challenge of diagnostic metagenomics. Expert Rev Mol Diagn. 2018;18(7):605-15. doi: 10.1080/14737159.2018.1487292. PubMed PMID: 29898605.

53. Simner PJ, Miller S, Carroll KC. Understanding the Promises and Hurdles of Metagenomic Next-Generation Sequencing as a Diagnostic Tool for Infectious Diseases. Clin Infect Dis. 2018;66(5):778-88. doi: 10.1093/cid/cix881. PubMed PMID: 29040428.

54. Besser J, Carleton HA, Gerner-Smidt P, Lindsey RL, Trees E. Next-generation sequencing technologies and their application to the study and control of bacterial infections. Clin Microbiol Infect. 2018;24(4):335-41. doi: 10.1016/j.cmi.2017.10.013. PubMed PMID: 29074157; PubMed Central PMCID: PMCPMC5857210.

55. Kafetzopoulou LE, Efthymiadis K, Lewandowski K, Crook A, Carter D, Osborne J, et al. Assessment of metagenomic Nanopore and Illumina sequencing for recovering whole genome sequences of chikungunya and dengue viruses directly from clinical samples. Euro Surveill. 2018;23(50). doi: 10.2807/1560-7917.ES.2018.23.50.1800228. PubMed PMID: 30563591; PubMed Central PMCID: PMCPMC6299504.

56. Batovska J, Lynch SE, Rodoni BC, Sawbridge TI, Cogan NO. Metagenomic arbovirus detection using MinION nanopore sequencing. J Virol Methods. 2017;249:79-84. doi: 10.1016/j.jviromet.2017.08.019. PubMed PMID: 28855093.

57. Quick J, Loman NJ, Duraffour S, Simpson JT, Severi E, Cowley L, et al. Real-time, portable genome sequencing for Ebola surveillance. Nature. 2016;530(7589):228-32. doi: 10.1038/nature16996. PubMed PMID: 26840485; PubMed Central PMCID: PMCPMC4817224.

58. Quick J, Grubaugh ND, Pullan ST, Claro IM, Smith AD, Gangavarapu K, et al. Multiplex PCR method for MinION and Illumina sequencing of Zika and other virus genomes directly from clinical samples. Nat Protoc. 2017;12(6):1261-76. doi: 10.1038/nprot.2017.066. PubMed PMID: 28538739; PubMed Central PMCID: PMCPMC5902022.

59. Faria NR, Kraemer MUG, Hill SC, Goes de Jesus J, Aguiar RS, lani FCM, et al. Genomic and epidemiological monitoring of yellow fever virus transmission potential. Science. 2018;361(6405):894-9. doi: 10.1126/science.aat7115. PubMed PMID: 30139911.

60. Kafetzopoulou LE, Pullan ST, Lemey P, Suchard MA, Ehichioya DU, Pahlmann M, et al. Metagenomic sequencing at the epicenter of the Nigeria 2018 Lassa fever outbreak. Science. 2019;363(6422):74-7. doi: 10.1126/science.aau9343. PubMed PMID: 30606844

61. Murray KO, Garcia MN, Yan C, Gorchakov R. Persistence of detectable immunoglobulin M antibodies up to 8 years after infection with West Nile virus. Am J Trop Med Hyg. 2013;89(5):996-1000. doi: 10.4269/ajtmh.13-0232. PubMed PMID: 24062481; PubMed Central PMCID: PMCPMC3820351.

62. Boldogh I, Albrecht T, Porter DD. Persistent Viral Infections. In: th, Baron S, editors. Medical Microbiology. Galveston (TX)1996.

63. Fechner K, Schäfer J, Wiegel C, Ludwig J, Münster P, Sharifi AR, et al. Distribution of Mycobacterium avium subsp. paratuberculosis in a Subclinical Naturally Infected German Fleckvieh Bull. Transbound Emerg Dis. 2015. doi: 10.1111/tbed.12459. PubMed PMID: 26671341.

64. Zheng T, Finn C, Parrett CJ, Dhume K, Hwang JH, Sidhom D, et al. A Rapid Blood Test To Determine the Active Status and Duration of Acute Viral Infection. ACS Infect Dis. 2017;3(11):866-73. doi: 10.1021/acsinfecdis.7b00137. PubMed PMID: 28918638.

65. Khatri M, Dwivedi V, Krakowka S, Manickam C, Ali A, Wang L, et al. Swine influenza H1N1 virus induces acute inflammatory immune responses in pig lungs: a potential animal model for human H1N1 influenza virus. J Virol. 2010;84(21):11210-8. doi: 10.1128/JVI.01211-10. PubMed PMID: 20719941; PubMed Central PMCID: PMCPMC2953174.

66. Wolfel R, Stoecker K, Fleischmann E, Gramsamer B, Wagner M, Molkenthin P, et al. Mobile diagnostics in outbreak response, not only for Ebola: a blueprint for a modular and robust field laboratory. Euro Surveill. 2015;20(44). doi: 10.2807/1560-7917.ES.2015.20.44.30055. PubMed PMID: 26559006. 
67. Faye O, Faye O, Soropogui B, Patel P, El Wahed AA, Loucoubar C, et al. Development and deployment of a rapid recombinase polymerase amplification Ebola virus detection assay in Guinea in 2015. Euro Surveill. 2015;20(44). doi: 10.2807/1560-7917.ES.2015.20.44.30053. PubMed PMID: 26558690.

68. Mondal D, Ghosh P, Khan MA, Hossain F, Bohlken-Fascher S, Matlashewski G, et al. Mobile suitcase laboratory for rapid detection of Leishmania donovani using recombinase polymerase amplification assay. Parasit Vectors. 2016;9(1):281. Epub 2016/05/15. doi: 10.1186/s13071-016-1572-8. PubMed PMID: 27177926; PubMed Central PMCID: PMCPMC4868004.

69. Abd El Wahed A, Weidmann M, Hufert FT. Diagnostics-in-a-Suitcase: Development of a portable and rapid assay for the detection of the emerging avian influenza A (H7N9) virus. J Clin Virol. 2015;69:16-21. doi: 10.1016/j.jcv.2015.05.004. PubMed PMID: 26209370.

70. ZIBRA Project. The Mobile Laboratory 2016 [cited 2018 December 10]. Available from: http://www.zibraproject.org/mobile/.

71. Praesens Foundation. Praesens Foundation; Field Diagnostics; Praesens Found 2019 [07.05.2019]. Available from: https://www.praesensfoundation.org.

72. Maillard PM. D'Ebola à Zika, un labo tout-terrain en Afrique de l'Ouest 2018. Available from: https://www.lemonde.fr/afrique/article/2018/09/12/d-ebola-a-zika-un-labo-tout-terrain-pour-lutter-contre-les-epidemies-enafrique-de-l-ouest_5354069_3212.html.

73. Drain PK, Hyle EP, Noubary F, Freedberg KA, Wilson D, Bishai WR, et al. Diagnostic point-of-care tests in resource-limited settings. Lancet Infect Dis. 2014;14(3):239-49. doi: 10.1016/S1473-3099(13)70250-0. PubMed PMID: 24332389; PubMed Central PMCID: PMCPMC4016042.

74. Shaw JLV. Practical challenges related to point of care testing. Pract Lab Med. 2016;4:22-9. doi: 10.1016/j.plabm.2015.12.002. PubMed PMID: 28856189; PubMed Central PMCID: PMCPMC5574506.

75. Price CP. Point of care testing. BMJ. 2001;322(7297):1285-8. PubMed PMID: 11375233; PubMed Central PMCID: PMCPMC1120384.

76. St John A, Price CP. Economic Evidence and Point-of-Care Testing. Clin Biochem Rev. 2013;34(2):61-74. PubMed PMID: 24151342; PubMed Central PMCID: PMCPMC3799220.

77. Pai NP, Vadnais C, Denkinger C, Engel N, Pai M. Point-of-care testing for infectious diseases: diversity, complexity, and barriers in low- and middle-income countries. PLoS Med. 2012;9(9):e1001306. doi: 10.1371/journal.pmed.1001306. PubMed PMID: 22973183; PubMed Central PMCID: PMCPMC3433407.

78. Pai NP, Wilkinson S, Deli-Houssein R, Vijh R, Vadnais C, Behlim T, et al. Barriers to Implementation of Rapid and Point-ofCare Tests for Human Immunodeficiency Virus Infection: Findings From a Systematic Review (1996-2014). Point Care. 2015;14(3):81-7. doi: 10.1097/POC.0000000000000056. PubMed PMID: 26366129; PubMed Central PMCID: PMCPMC4549862.

79. Rasmussen DN, Unger HW, Bjerregaard-Andersen M, da Silva Te D, Vieira N, Oliveira I, et al. Political instability and supplyside barriers undermine the potential for high participation in HIV testing for the prevention of mother-to-child transmission in Guinea-Bissau: A retrospective cross-sectional study. PLoS One. 2018;13(8):e0199819. doi: 10.1371/journal.pone.0199819. PubMed PMID: 30067793; PubMed Central PMCID: PMCPMC6070169.

80. Engel N, Yellappa V, Davids M, Dheda K, Pai NP, Pai M, editors. Barriers to Point of Care Testing in India and South Africa2018; Cham: Springer International Publishing.

81. Boadu NY, Amuasi J, Ansong D, Einsiedel E, Menon D, Yanow SK. Challenges with implementing malaria rapid diagnostic tests at primary care facilities in a Ghanaian district: a qualitative study. Malar J. 2016;15:126. doi: 10.1186/s12936-0161174-0. PubMed PMID: 26921263; PubMed Central PMCID: PMCPMC4769585.

82. Cummins BM, Ligler FS, Walker GM. Point-of-care diagnostics for niche applications. Biotechnology advances. 2016;34(3):161-76. Epub 02/01. doi: 10.1016/j.biotechadv.2016.01.005. PubMed PMID: 26837054.

83. Hardy V, Thompson M, Alto W, Keppel GA, Hornecker J, Linares A, et al. Exploring the barriers and facilitators to use of point of care tests in family medicine clinics in the United States. BMC Fam Pract. 2016;17(1):149. doi: 10.1186/s12875016-0549-1. PubMed PMID: 27809865; PubMed Central PMCID: PMCPMC5093922.

84. Doctors Without Borders. NEGLECTED TROPICAL DISEASES: NEW MSF REPORT HIGHLIGHTS HOW TO BREAK $\begin{array}{llllll}\text { CYCLE OF } & \text { NEGLECT } & 2012 & \text { [01.05.2019]. }\end{array}$ https://www.who.int/neglected_diseases/NTD_PR_FINAL_Jun2012_ENG.pdf?ua=1.

85. Gebreyes WA, Dupouy-Camet J, Newport MJ, Oliveira CJ, Schlesinger LS, Saif YM, et al. The global one health paradigm: challenges and opportunities for tackling infectious diseases at the human, animal, and environment interface in lowresource settings. PLoS Negl Trop Dis. 2014;8(11):e3257. doi: 10.1371/journal.pntd.0003257. PubMed PMID: 25393303; PubMed Central PMCID: PMCPMC4230840.

86. Kurpiers LA, Schulte-Herbrüggen B, Ejotre I, Reeder DM. Bushmeat and Emerging Infectious Diseases: Lessons from Africa. In: Angelici FM, editor. Problematic Wildlife: A Cross-Disciplinary Approach. Cham: Springer International Publishing; 2016. p. 507-51.

87. Wilcox BA. Forests and emerging infectious diseases of humans. Unasylva. 2006;224(57):11-9.

88. Kahn LH. Confronting zoonoses, linking human and veterinary medicine. Emerg Infect Dis. 2006;12(4):556-61. doi: 10.3201/eid1204.050956. PubMed PMID: 16704801; PubMed Central PMCID: PMCPMC3294691.

89. Whitfield $\mathrm{Y}$, Johnson K, Hobbs L, Middleton D, Dhar B, Vrbova L. Descriptive study of enteric zoonoses in Ontario, Canada, from 2010 - 2012. Bmc Public Health. 2017;17(1):217. doi: 10.1186/s12889-017-4135-9. PubMed PMID: 28222719; PubMed Central PMCID: PMCPMC5320741. 
90. Zumla A, Goodfellow I, Kasolo F, Ntoumi F, Buchy P, Bates M, et al. Zika virus outbreak and the case for building effective and sustainable rapid diagnostics laboratory capacity globally. Int J Infect Dis. 2016;45:92-4. doi: 10.1016/j.ijid.2016.02.1007. PubMed PMID: 26952389.

91. Cohen GM. Access to diagnostics in support of HIVIAIDS and tuberculosis treatment in developing countries. AIDS. 2007;21 Suppl 4:S81-7. doi: 10.1097/01.aids.0000279710.47298.5c. PubMed PMID: 17620757.

92. Alabi AS, Traore AN, Loembe MM, Ateba-Ngoa U, Frank M, Adegnika AA, et al. Enhanced laboratory capacity development: a boost for effective tuberculosis control in resource-limited settings. Int J Infect Dis. 2017;56:81-4. doi: 10.1016/j.ijid.2016.11.404. PubMed PMID: 27888000.

93. Schroeder LF, Amukele T. Medical Laboratories in Sub-Saharan Africa That Meet International Quality Standards. American Journal of Clinical Pathology. 2014;141(6):791-5. doi: 10.1309/ajcpq5ktkagsscfn.

94. Kouadio IK, Aljunid S, Kamigaki T, Hammad K, Oshitani H. Infectious diseases following natural disasters: prevention and control measures. Expert Rev Anti Infect Ther. 2012;10(1):95-104. doi: 10.1586/eri.11.155. PubMed PMID: 22149618.

95. Brock TK, Mecozzi DM, Sumner S, Kost GJ. Evidence-based point-of-care tests and device designs for disaster preparedness. Am J Disaster Med. 2010;5(5):285-94. PubMed PMID: 21162410; PubMed Central PMCID: PMCPMC3074570.

96. World Health Organization. Communicable diseases following natural disasters 2006 [18.04.2019]. Available from: https://www.who.int/diseasecontrol_emergencies/guidelines/CD_Disasters_26_06.pdf?ua=1\%20.

97. Kost GJ, Tran NK, Tuntideelert M, Kulrattanamaneeporn S, Peungposop N. Katrina, the tsunami, and point-of-care testing: optimizing rapid response diagnosis in disasters. Am J Clin Pathol. 2006;126(4):513-20. doi: 10.1309/NWU5E6TOL4PFCBD9. PubMed PMID: 16938656.

98. Thiermann A. Emerging diseases and implications for global trade. Rev Sci Tech Oie. 2004;23(2):701-7. doi: DOI 10.20506/rst.23.2.1509. PubMed PMID: WOS:000226229400021.

99. Wang LF, Crameri G. Emerging zoonotic viral diseases. Rev Sci Tech. 2014;33(2):569-81. PubMed PMID: 25707184.

100. World Organization for Animal Health. OIE-Listed diseases, infections and infestations in force in 20192019 [09.04.2019]. Available from: http://www.oie.int/animal-health-in-the-world/oie-listed-diseases-2019/.

101. Lanata CF, Fischer-Walker CL, Olascoaga AC, Torres CX, Aryee MJ, Black RE, et al. Global causes of diarrheal disease mortality in children <5 years of age: a systematic review. PLoS One. 2013;8(9):e72788. doi: 10.1371/journal.pone.0072788. PubMed PMID: 24023773; PubMed Central PMCID: PMCPMC3762858.

102. Destoumieux-Garzon D, Mavingui P, Boetsch G, Boissier J, Darriet F, Duboz P, et al. The One Health Concept: 10 Years Old and a Long Road Ahead. Front Vet Sci. 2018;5:14. doi: 10.3389/fvets.2018.00014. PubMed PMID: 29484301; PubMed Central PMCID: PMCPMC5816263.

103. Manning EJ, Collins MT. Mycobacterium avium subsp. paratuberculosis: pathogen, pathogenesis and diagnosis. Rev Sci Tech. 2001;20(1):133-50. PubMed PMID: 11288509

104. Chi J, VanLeeuwen JA, Weersink A, Keefe GP. Direct production losses and treatment costs from bovine viral diarrhoea virus, bovine leukosis virus, Mycobacterium avium subspecies paratuberculosis, and Neospora caninum. Prev Vet Med. 2002;55(2):137-53. PubMed PMID: 12350317.

105. Whitlock RH, Buergelt C. Preclinical and clinical manifestations of paratuberculosis (including pathology). Vet Clin North Am Food Anim Pract. 1996;12(2):345-56. PubMed PMID: 8828109.

106. Corbett CS, De Buck J, Orsel K, Barkema HW. Fecal shedding and tissue infections demonstrate transmission of Mycobacterium avium subsp. paratuberculosis in group-housed dairy calves. Vet Res. 2017;48(1):27. doi: 10.1186/s13567017-0431-8. PubMed PMID: 28454560; PubMed Central PMCID: PMCPMC5410103.

107. Angelidou E, Kostoulas P, Leontides L. Bayesian estimation of sensitivity and specificity of a commercial serum/milk ELISA against the Mycobacterium avium subsp. Paratuberculosis (MAP) antibody response for each lactation stage in Greek dairy sheep. Prev Vet Med. 2016;124:102-5. doi: 10.1016/j.prevetmed.2015.12.011. PubMed PMID: 26754926.

108. Zhang MZ, Zhang S. An efficient DNA extraction method for polymerase chain reaction-based detection of Mycobacterium avium subspecies paratuberculosis in bovine fecal samples. J Vet Diagn Invest. 2011;23(1):41-8. doi: 10.1177/104063871102300106. PubMed PMID: 21217026

109. McNeil MR, Brennan PJ. Structure, function and biogenesis of the cell envelope of mycobacteria in relation to bacterial physiology, pathogenesis and drug resistance; some thoughts and possibilities arising from recent structural information. Res Microbiol. 1991;142(4):451-63. PubMed PMID: 1871433.

110. Amaro A, Duarte E, Amado A, Ferronha H, Botelho A. Comparison of three DNA extraction methods for Mycobacterium bovis, Mycobacterium tuberculosis and Mycobacterium avium subsp. avium. Lett Appl Microbiol. 2008;47(1):8-11. doi: 10.1111/j.1472-765X.2008.02372.x. PubMed PMID: 18498320.

111. Jamal SM, Belsham GJ. Foot-and-mouth disease: past, present and future. Vet Res. 2013;44:116. doi: 10.1186/1297-971644-116. PubMed PMID: 24308718; PubMed Central PMCID: PMCPMC4028749.

112. Schaller H, Forss S, Strebel K, Beck E, Böhm HO, Leban J, et al., editors. Foot and Mouth Disease Virus: Genome Organization, Antigenic Variation, and New Approaches to a Safe Vaccine. The Impact of Gene Transfer Techniques in Eukaryotic Cell Biology; 1984 1984//; Berlin, Heidelberg: Springer Berlin Heidelberg.

113. Knight-Jones TJ, Rushton J. The economic impacts of foot and mouth disease - what are they, how big are they and where do they occur? Prev Vet Med. 2013;112(3-4):161-73. doi: 10.1016/j.prevetmed.2013.07.013. PubMed PMID: 23958457; PubMed Central PMCID: PMCPMC3989032. 
114. Roeder PL, Le Blanc Smith PM. Detection and typing of foot-and-mouth disease virus by enzyme-linked immunosorbent assay: a sensitive, rapid and reliable technique for primary diagnosis. Research in veterinary science. 1987;43(2):225-32. PubMed PMID: 2825310.

115. Abd El Wahed A, El-Deeb A, El-Tholoth M, Abd El Kader H, Ahmed A, Hassan S, et al. A portable reverse transcription recombinase polymerase amplification assay for rapid detection of foot-and-mouth disease virus. PLoS One. 2013;8(8):e71642. doi: 10.1371/journal.pone.0071642. PubMed PMID: 23977101; PubMed Central PMCID: PMCPMC3748043.

116. Rasmussen TB, Uttenthal A, de Stricker K, Belak S, Storgaard T. Development of a novel quantitative real-time RT-PCR assay for the simultaneous detection of all serotypes of foot-and-mouth disease virus. Arch Virol. 2003;148(10):2005-21. doi: 10.1007/s00705-003-0145-2. PubMed PMID: 14551821.

117. Bachanek-Bankowska K, Mero HR, Wadsworth J, Mioulet V, Sallu R, Belsham GJ, et al. Development and evaluation of tailored specific real-time RT-PCR assays for detection of foot-and-mouth disease virus serotypes circulating in East Africa. J Virol Methods. 2016;237:114-20. doi: 10.1016/j.jviromet.2016.08.002. PubMed PMID: 27575682.

118. Wang H, Hou P, Zhao G, Yu L, Gao YW, He H. Development and evaluation of serotype-specific recombinase polymerase amplification combined with lateral flow dipstick assays for the diagnosis of foot-and-mouth disease virus serotype $\mathrm{A}$, $\mathrm{O}$ and Asia1. BMC Vet Res. 2018;14(1):359. doi: 10.1186/s12917-018-1644-4. PubMed PMID: 30458768; PubMed Central PMCID: PMCPMC6245561.

119. Waggoner JJ, Pinsky BA. Zika Virus: Diagnostics for an Emerging Pandemic Threat. J Clin Microbiol. 2016;54(4):860-7. doi: 10.1128/JCM.00279-16. PubMed PMID: 26888897; PubMed Central PMCID: PMCPMC4809954.

120. International Committee on Taxonomy of Viruses. ICTV Master Species List 2018b.v1 2018 [16.04.2019]. Available from: https://talk.ictvonline.org/files/master-species-lists/m/msl/8266.

121. World Health Organization. Pandemic, epidemic diseases 2019 [09.04.2019]. Available from: https://www.who.int/emergencies/diseases/en/.

122. World Health Organization. Neglected tropical diseases 2019 [09.04.2019]. Available from: https://www.who.int/neglected diseases/diseases/en/.

123. Heinz FX, Stiasny K. The Antigenic Structure of Zika Virus and Its Relation to Other Flaviviruses: Implications for Infection and Immunoprophylaxis. Microbiol Mol Biol Rev. 2017;81(1). doi: 10.1128/MMBR.00055-16. PubMed PMID: 28179396; PubMed Central PMCID: PMCPMC5312239.

124. World Health Organization. Progress toward discovery of Zika virus vaccines and therapeutics 2018 [01.04.2019]. Available from: https://www.who.int/emergencies/diseases/zika/discovery-of-vaccines/en/.

125. World Health Organization. List of Blueprint priority diseases 2018 [01.04.2019]. Available from: https://www.who.int/blueprint/priority-diseases/en/

126. Viral Zone Swiss Institute of Bioinformatics. Zika Virus 2016 [11.04.2019]. Available from: https://viralzone.expasy.org/6756?outline=all_by_species.

127. Terzian ACB, Zini N, Sacchetto L, Rocha RF, Parra MCP, Del Sarto JL, et al. Evidence of natural Zika virus infection in neotropical non-human primates in Brazil. Sci Rep. 2018;8(1):16034. doi: 10.1038/s41598-018-34423-6. PubMed PMID: 30375482; PubMed Central PMCID: PMCPMC6207778.

128. Bueno MG, Martinez N, Abdalla L, Duarte Dos Santos CN, Chame M. Animals in the Zika Virus Life Cycle: What to Expect from Megadiverse Latin American Countries. PLoS Negl Trop Dis. 2016;10(12):e0005073. doi: 10.1371/journal.pntd.0005073. PubMed PMID: 28005902; PubMed Central PMCID: PMCPMC5179043.

129. United States Centers for Disease Control and Prevention. Zika and Animals 2018 [28.04.2019]. Available from: https://www.cdc.gov/zika/transmission/qa-animals.html.

130. Patterson J, Sammon M, Garg M. Dengue, Zika and Chikungunya: Emerging Arboviruses in the New World. West J Emerg Med. 2016;17(6):671-9. doi: 10.5811/westjem.2016.9.30904. PubMed PMID: 27833670; PubMed Central PMCID: PMCPMC5102589 all affiliations, funding sources and financial or management relationships that could be perceived as potential sources of bias. The authors disclosed none.

131. Ciota AT, Kramer LD. Vector-virus interactions and transmission dynamics of West Nile virus. Viruses. 2013;5(12):302147. doi: 10.3390/v5123021. PubMed PMID: 24351794; PubMed Central PMCID: PMCPMC3967159.

132. Paixao ES, Teixeira MG, Rodrigues LC. Zika, chikungunya and dengue: the causes and threats of new and re-emerging arboviral diseases. BMJ Glob Health. 2018;3(Suppl 1):e000530. doi: 10.1136/bmjgh-2017-000530. PubMed PMID: 29435366; PubMed Central PMCID: PMCPMC5759716.

133. Turtle L, Griffiths MJ, Solomon T. Encephalitis caused by flaviviruses. QJM. 2012;105(3):219-23. doi: 10.1093/qjmed/hcs013. PubMed PMID: 22367423; PubMed Central PMCID: PMCPMC3285924.

134. Gould EA, Solomon T. Pathogenic flaviviruses. Lancet. 2008;371(9611):500-9. doi: 10.1016/S0140-6736(08)60238-X. PubMed PMID: 18262042

135. Mansuy JM, Mengelle C, Pasquier C, Chapuy-Regaud S, Delobel P, Martin-Blondel G, et al. Zika Virus Infection and Prolonged Viremia in Whole-Blood Specimens. Emerg Infect Dis. 2017;23(5):863-5. doi: 10.3201/eid2305.161631. PubMed PMID: 28257281; PubMed Central PMCID: PMCPMC5403064.

136. van Meer MPA, Mogling R, Klaasse J, Chandler FD, Pas SD, van der Eijk AA, et al. Re-evaluation of routine dengue virus serology in travelers in the era of Zika virus emergence. J Clin Virol. 2017;92:25-31. doi: 10.1016/j.jcv.2017.05.001. PubMed PMID: 28505571. 
137. Liu L, Li Y, Li S, Hu N, He Y, Pong R, et al. Comparison of next-generation sequencing systems. J Biomed Biotechnol. 2012;2012:251364. doi: 10.1155/2012/251364. PubMed PMID: 22829749; PubMed Central PMCID: PMCPMC3398667.

138. Schmaljohn AL, McClain D. Alphaviruses (Togaviridae) and Flaviviruses (Flaviviridae). In: th, Baron S, editors. Medical Microbiology. Galveston (TX)1996.

139. Calisher $\mathrm{CH}$, Karabatsos N, Dalrymple JM, Shope RE, Porterfield JS, Westaway EG, et al. Antigenic relationships between flaviviruses as determined by cross-neutralization tests with polyclonal antisera. J Gen Virol. 1989;70 ( Pt 1):37-43. doi: 10.1099/0022-1317-70-1-37. PubMed PMID: 2543738.

140. Barba-Spaeth G, Dejnirattisai W, Rouvinski A, Vaney M-C, Medits I, Sharma A, et al. Structural basis of potent Zika-dengue virus antibody cross-neutralization. Nature. 2016;536:48. doi: 10.1038/nature18938.

141. De S, Kaur G, Roy A, Dogra G, Kaushik R, Yadav P, et al. A Simple Method for the Efficient Isolation of Genomic DNA from Lactobacilli Isolated from Traditional Indian Fermented Milk (dahi). Indian J Microbiol. 2010;50(4):412-8. doi: 10.1007/s12088-011-0079-4. PubMed PMID: 22282608; PubMed Central PMCID: PMCPMC3209835.

142. Brunzel NA. Fundamentals of urine and body fluid analysis. 4 ed. St. Louis, Missouri, USA: Elsevier; 2018.

143. Gunaratna G, Manamperi A, Bohlken-Fascher S, Wickremasinge R, Gunawardena K, Yapa B, et al. Evaluation of rapid extraction and isothermal amplification techniques for the detection of Leishmania donovani DNA from skin lesions of suspected cases at the point of need in Sri Lanka. Parasit Vectors. 2018;11(1):665. doi: 10.1186/s13071-018-3238-1. PubMed PMID: 30577826; PubMed Central PMCID: PMCPMC6303884.

144. Weidmann M, Faye O, Faye O, Abd El Wahed A, Patel P, Batejat C, et al. Development of Mobile Laboratory for Viral Hemorrhagic Fever Detection in Africa. J Infect Dis. 2018;218(10):1622-30. doi: 10.1093/infdis/jiy362. PubMed PMID: 29917112; PubMed Central PMCID: PMCPMC6173574.

145. Schlottau K, Freuling CM, Muller T, Beer M, Hoffmann B. Development of molecular confirmation tools for swift and easy rabies diagnostics. Virol J. 2017;14(1):184. doi: 10.1186/s12985-017-0853-y. PubMed PMID: 28938887; PubMed Central PMCID: PMCPMC5610444.

146. Xuan J, Yu Y, Qing T, Guo L, Shi L. Next-generation sequencing in the clinic: promises and challenges. Cancer Lett. 2013;340(2):284-95. doi: 10.1016/j.canlet.2012.11.025. PubMed PMID: 23174106; PubMed Central PMCID: PMCPMC5739311.

147. Quiagen Hilden. REPLI-g Mini Kit For highly uniform whole genome amplification from small or precious samples 2019 [03.05.2019]. Available from: https://www.qiagen.com/us/shop/repli-g-mini-kit/\#orderinginformation.

148. You SH, Pyo HM, Lee SY, Ko MK, Choi JH, Shin SH, et al. Complete Genome Sequence of Foot-and-Mouth Disease Virus Serotype SAT3 Zimbabwe/4/81. Genome Announc. 2016;4(4). doi: 10.1128/genomeA.00801-16. PubMed PMID: 27563037; PubMed Central PMCID: PMCPMC5000821.

149. Zhu YY, Machleder EM, Chenchik A, Li R, Siebert PD. Reverse transcriptase template switching: a SMART approach for full-length cDNA library construction. Biotechniques. 2001;30(4):892-7. doi: 10.2144/01304pf02. PubMed PMID: 11314272.

150. Nakano K, Shiroma A, Shimoji M, Tamotsu H, Ashimine N, Ohki S, et al. Advantages of genome sequencing by long-read sequencer using SMRT technology in medical area. Hum Cell. 2017;30(3):149-61. doi: 10.1007/s13577-017-0168-8. PubMed PMID: 28364362; PubMed Central PMCID: PMCPMC5486853.

151. Tyler AD, Mataseje L, Urfano CJ, Schmidt L, Antonation KS, Mulvey MR, et al. Evaluation of Oxford Nanopore's MinION Sequencing Device for Microbial Whole Genome Sequencing Applications. Scientific Reports. 2018;8(1):10931. doi: 10.1038/s41598-018-29334-5

152. Beck C, Jimenez-Clavero MA, Leblond A, Durand B, Nowotny N, Leparc-Goffart I, et al. Flaviviruses in Europe: complex circulation patterns and their consequences for the diagnosis and control of West Nile disease. Int $\mathrm{J}$ Environ Res Public Health. 2013;10(11):6049-83. doi: 10.3390/ijerph10116049. PubMed PMID: 24225644; PubMed Central PMCID: PMCPMC3863887.

153. Dejnirattisai W, Wongwiwat W, Supasa S, Zhang X, Dai X, Rouvinski A, et al. A new class of highly potent, broadly neutralizing antibodies isolated from viremic patients infected with dengue virus. Nature Immunology. 2014;16:170. doi: 10.1038/ni.3058 https://www.nature.com/articles/ni.3058\#supplementary-information.

154. Rastogi M, Sharma N, Singh SK. Flavivirus NS1: a multifaceted enigmatic viral protein. Virol J. 2016;13:131. doi: 10.1186/s12985-016-0590-7. PubMed PMID: 27473856; PubMed Central PMCID: PMCPMC4966872.

155. Wong SJ, Boyle RH, Demarest VL, Woodmansee AN, Kramer LD, Li H, et al. Immunoassay targeting nonstructural protein 5 to differentiate West Nile virus infection from dengue and St. Louis encephalitis virus infections and from flavivirus vaccination. J Clin Microbiol. 2003;41(9):4217-23. PubMed PMID: 12958248; PubMed Central PMCID: PMCPMC193845.

156. Rothman AL, Kurane I, Lai CJ, Bray M, Falgout B, Men R, et al. Dengue virus protein recognition by virus-specific murine CD8+ cytotoxic T lymphocytes. J Virol. 1993;67(2):801-6. PubMed PMID: 7678307; PubMed Central PMCID: PMCPMC237433.

157. Premkumar L, Collins M, Graham S, Liou GA, Lopez CA, Jadi R, et al. Development of envelope protein antigens to serologically differentiate Zika from dengue virus infection. J Clin Microbiol. 2017. doi: 10.1128/JCM.01504-17. PubMed PMID: 29263206.

158. Tsai WY, Youn HH, Brites C, Tsai JJ, Tyson J, Pedroso C, et al. Distinguishing Secondary Dengue Virus Infection From Zika Virus Infection With Previous Dengue by a Combination of 3 Simple Serological Tests. Clin Infect Dis. 2017;65(11):1829-36. doi: 10.1093/cid/cix672. PubMed PMID: 29020159.

159. Balmaseda A, Stettler K, Medialdea-Carrera R, Collado D, Jin X, Zambrana JV, et al. Antibody-based assay discriminates Zika virus infection from other flaviviruses. Proc Natl Acad Sci U S A. 2017;114(31):8384-9. doi: 10.1073/pnas.1704984114. PubMed PMID: 28716913; PubMed Central PMCID: PMCPMC5547631. 
160. Heffron AS, Mohr EL, Baker D, Haj AK, Buechler CR, Bailey A, et al. Antibody responses to Zika virus proteins in pregnant and non-pregnant macaques. PLoS Negl Trop Dis. 2018;12(11):e0006903. doi: 10.1371/journal.pntd.0006903. PubMed PMID: 30481182; PubMed Central PMCID: PMCPMC6286021 following competing interests. This manuscript describes the use of a platform provided on an early-access basis by Roche Sequencing Solutions. While scientists from Roche were involved in the experimental design and data analysis, the manuscript was prepared independently from Roche and did not require pre-approval from Roche prior to submission. RSP, EB, HL, JP, and JCT are employed by Roche Sequencing Solutions.

161. Mishra N, Caciula A, Price A, Thakkar R, Ng J, Chauhan LV, et al. Diagnosis of Zika Virus Infection by Peptide Array and Enzyme-Linked Immunosorbent Assay. MBio. 2018;9(2). doi: 10.1128/mBio.00095-18. PubMed PMID: 29511073; PubMed Central PMCID: PMCPMC5844993.

162. Gyurech D, Schilling J, Schmidt-Chanasit J, Cassinotti P, Kaeppeli F, Dobec M. False positive dengue NS1 antigen test in a traveller with an acute Zika virus infection imported into Switzerland. Swiss Med Wkly. 2016;146:w14296. doi: 10.4414/smw.2016.14296. PubMed PMID: 26859285.

163. Matheus S, Boukhari R, Labeau B, Ernault V, Bremand L, Kazanji M, et al. Specificity of Dengue NS1 Antigen in Differential Diagnosis of Dengue and Zika Virus Infection. Emerging infectious diseases. 2016;22(9):1691-3. doi: 10.3201/eid2209.160725. PubMed PMID: 27347853; PubMed Central PMCID: PMC4994358.

164. Ladner RC. Mapping the epitopes of antibodies. Biotechnol Genet Eng Rev. 2007;24:1-30. PubMed PMID: 18059626.

165. Wenschuh H, Volkmer-Engert R, Schmidt M, Schulz M, Schneider-Mergener J, Reineke U. Coherent membrane supports for parallel microsynthesis and screening of bioactive peptides. Biopolymers. 2000;55(3):188-206. doi: 10.1002/10970282(2000)55:3<188::AID-BIP20>3.0.CO;2-T. PubMed PMID: 11074414.

166. Lin J, Bardina L, Shreffler WG, Andreae DA, Ge Y, Wang J, et al. Development of a novel peptide microarray for large-scale epitope mapping of food allergens. J Allergy Clin Immunol. 2009;124(2):315-22, 22 e1-3. doi: 10.1016/j.jaci.2009.05.024. PubMed PMID: 19577281; PubMed Central PMCID: PMCPMC2757036.

167. Hecker M, Fitzner B, Wendt M, Lorenz P, Flechtner K, Steinbeck F, et al. High-Density Peptide Microarray Analysis of IgG Autoantibody Reactivities in Serum and Cerebrospinal Fluid of Multiple Sclerosis Patients. Mol Cell Proteomics. 2016;15(4):1360-80. doi: 10.1074/mcp.M115.051664. PubMed PMID: 26831522; PubMed Central PMCID: PMCPMC4824861.

168. Hansen LB, Buus S, Schafer-Nielsen C. Identification and mapping of linear antibody epitopes in human serum albumin using high-density Peptide arrays. PLoS One. 2013;8(7):e68902. doi: 10.1371/journal.pone.0068902. PubMed PMID: 23894373; PubMed Central PMCID: PMCPMC3720873.

169. Mumey BM, Bailey BW, Kirkpatrick B, Jesaitis AJ, Angel T, Dratz EA. A new method for mapping discontinuous antibody epitopes to reveal structural features of proteins. J Comput Biol. 2003;10(3-4):555-67. doi: 10.1089/10665270360688183. PubMed PMID: 12935344

170. Zhu H, Luo H, Yan M, Zuo X, Li QZ. Autoantigen Microarray for High-throughput Autoantibody Profiling in Systemic Lupus Erythematosus. Genomics Proteomics Bioinformatics. 2015;13(4):210-8. doi: 10.1016/j.gpb.2015.09.001. PubMed PMID: 26415621; PubMed Central PMCID: PMCPMC4610965.

171. Pamelard F, Even G, Apostol C, Preda C, Dhaenens C, Fafeur V, et al. PASE: a web-based platform for peptide/protein microarray experiments. Methods Mol Biol. 2009;570:413-30. doi: 10.1007/978-1-60327-394-7_24. PubMed PMID: 19649610.

172. Renard BY, Lower M, Kuhne Y, Reimer U, Rothermel A, Tureci O, et al. rapmad: Robust analysis of peptide microarray data. BMC Bioinformatics. 2011;12:324. doi: 10.1186/1471-2105-12-324. PubMed PMID: 21816082; PubMed Central PMCID: PMCPMC3174949.

173. Imholte GC, Sauteraud R, Korber B, Bailer RT, Turk ET, Shen X, et al. A computational framework for the analysis of peptide microarray antibody binding data with application to HIV vaccine profiling. J Immunol Methods. 2013;395(1-2):1-13. doi: 10.1016/j.jim.2013.06.001. PubMed PMID: 23770318; PubMed Central PMCID: PMCPMC3999921.

174. Mukherjee S. Emerging Infectious Diseases: Epidemiological Perspective. Indian J Dermatol. 2017;62(5):459-67. doi: 10.4103/ijd.IJD_379_17. PubMed PMID: 28979007; PubMed Central PMCID: PMCPMC5618832. 


\section{Chapter VIII: Appendix}

Supplementary Material

Development of Rapid Extraction Method of Mycobacterium avium subspecies paratuberculosis DNA from Bovine Stool Samples

Sören Hansen ${ }^{1}$, Marco Roller ${ }^{2}$, Lamia Alslim ${ }^{1}$, Susanne Böhlken-Fascher ${ }^{1}$, Kim

Fechner $^{1}$, Claus-Peter Czerny ${ }^{1}$, Ahmed Abd El Wahed ${ }^{1 *}$

1) Division of Microbiology and Animal Hygiene, Department of Animal Sciences, Faculty of Agricultural Sciences, University of Goettingen, Goettingen, Germany

2) Zoological and Botanical Garden Wilhelma, Stuttgart, Germany

${ }^{*}$ Corresponding Author 


\section{Supplementary Figure S1}
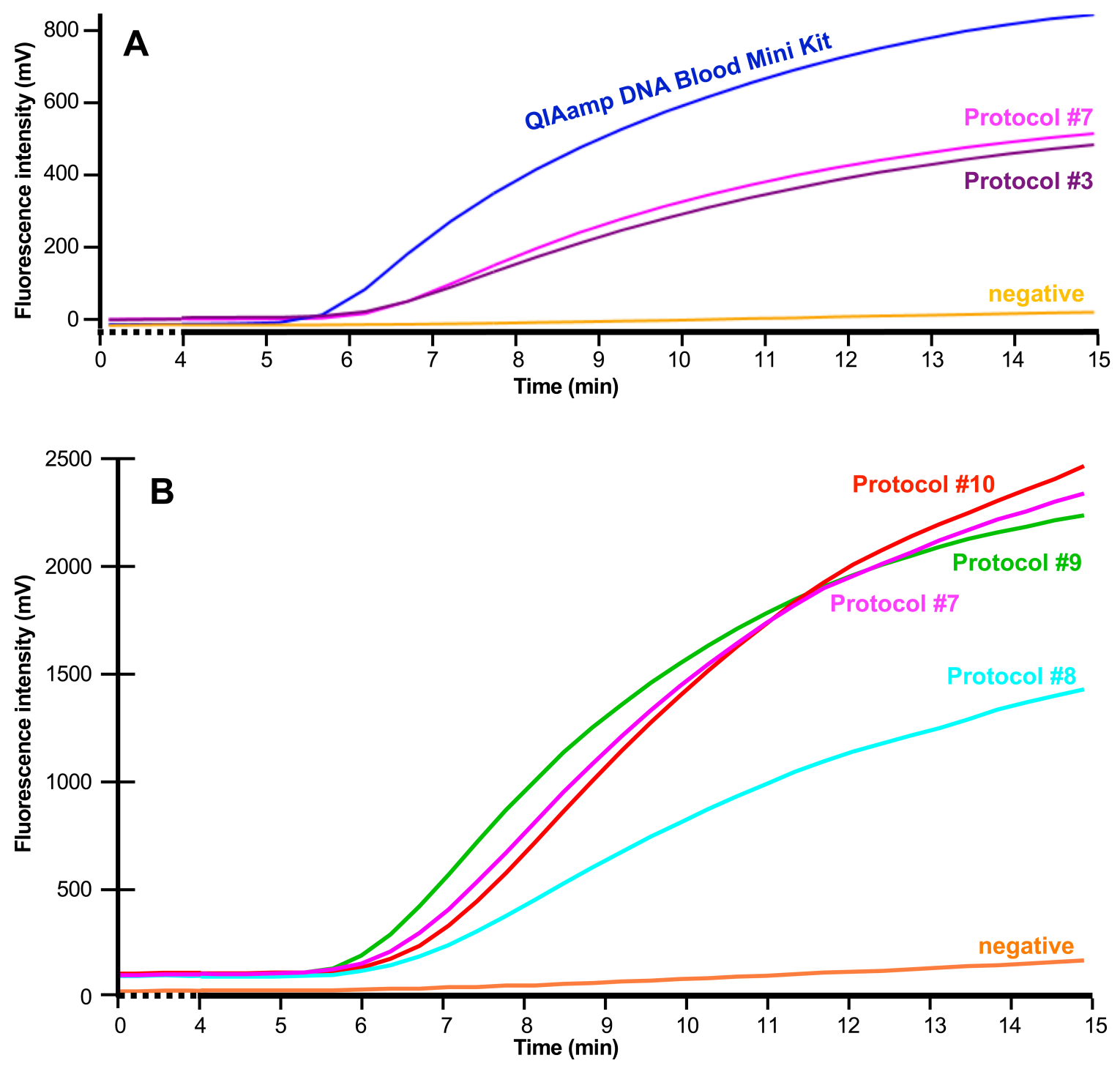

Figure S1. A: RPA results of DNA extracted either by the QIAamp DNA Mini Blood Kit (blue) or the MAP SpeedXtract protocol with use of Proteinase K (purple) and without (pink).

B: Performance of the DNA extraction protocols \#7 to \#10. 


\section{Supplementary File S1}

\section{MAP SpeedXtract Protocol \#10}

\section{Preparations:}

O Switch on the heat block and adjust the temperature to $95^{\circ} \mathrm{C}$. Please be sure that the heat block reach $95^{\circ} \mathrm{C}$ before starting with the extraction protocol.

O Prepare for each sample two $1.5 \mathrm{ml}$ tubes containing $40 \mu \mathrm{l}$ water, two Precellys SK 38 tubes and two appropriate tubes for storage. Label them with the sample ID.

O Vortex SpeedXtract Suspension A for 30 seconds to resuspend magnetic particles.

\section{SpeedXtract protocol:}

O Add $100 \mathrm{mg}$ of the sample to $500 \mu \mathrm{l}$ Buffer SL in a Precellys SK 38 tube.

O As an extraction control, please add $500 \mu \mathrm{l}$ Buffer SL in a Precellys SK 38 tube.

O Place tubes on a Vortex and mix for $1 \mathrm{~min}$

$\bigcirc 60 \mu \mathrm{l}$ of Suspension A to the sample.

O Mix well for 10 seconds by vortexing or inverting.

O Incubate tubes at $95^{\circ} \mathrm{C}$ for 15 minutes. Remove the tube every two minutes from the heat block and vortex.

Remove sample tubes from heat block after 15 minutes and either shake down or tap on a bench to remove condensate from the lid.

O Transfer sample tubes to a magnetic stand and incubate at room temperature for 2 minutes.

O Carefully open sample tubes and transfer $10 \mu \mathrm{l}$ of the supernatant to a $1.5 \mathrm{ml}$ tube containing $40 \mu \mathrm{l}$ water to dilute the sample 1:5.

O Put dilute sample direct in RPA and store the rest of the sample at $-20^{\circ} \mathrm{C}$ in an appropriate tube. 


\section{Supplementary Material}

Serotyping of foot-and-mouth disease virus using oxford nanopore sequencing

Sören Hansen ${ }^{1 *}$, Veronika Dill ${ }^{2}$, Mohamed A. Shalaby ${ }^{3}$, Michael Eschbaumer ${ }^{2}$, Susanne Böhlken-Fascher ${ }^{1}$, Bernd Hoffmann ${ }^{2}$, Claus-Peter Czerny ${ }^{1}$, Ahmed Abd El Wahed $^{1}$

\footnotetext{
1) Division of Microbiology and Animal Hygiene, University of Goettingen, Burckhardtweg 2, D-37077 Goettingen Greifswald-Insel Riems, Germany.

3) Department of Virology, Faculty of Veterinary Medicine, Cairo University, 12211, Giza, Egypt

${ }^{*}$ Corresponding Author
}

2) Institute of Diagnostic Virology, Friedrich-Loeffler-Institut, Federal Research Institute for Animal Health, Suedufer 10, D-17493 


\section{Additional file 1: Foot and Mouth Disease Virus Direct cDNA native Barcoding Protocol for library preparation}

\section{Before Start prepare the following:}

Direct cDNA Sequencing Kit (Oxford Nanopore, Oxford, UK; SQK-DCS108)

Native Barcoding Kit 1D (Oxford Nanopore, Oxford, UK; EXP-NBD103)

VN primer, VNP (5' - 5phos/

ACTTGCCTGTCGCTCTATCTTCTTTTTTTTTTTTTTTTTTTTVN - 3') (TIB MOLBIOL, Berlin, Germany)

Strand Switching primer, SSP (5'-TTTCTGTTGGTGCTGATATTGCTGCCATTACGGCC mGmGmG with 2' O-methyl RNA bases-3') (TIB MOLBIOL, Berlin, Germany)

PR2 Primer 5'-TTTCTgTTggTgCTgATATTgC-3' (TIB MOLBIOL, Berlin, Germany) $200 \mu \mathrm{l}$ tubes (Eppendorf AG, Hamburg, Germany; 022510509)

1.5 ml Eppendorf DNA LoBind tubes (Eppendorf AG, Hamburg, Germany; 022431021)

Nuclease-free water

Freshly prepared $70 \%$ ethanol in nuclease free water

LongAmp Taq 2X Master Mix (New England Biolabs, Ipswich, MA, USA; M0287)

Agencourt AMPure XP beads (Beckman Coulter, Brea, CA, USA; A63880)

SuperScript IV reverse transcriptase, 5x RT buffer and 100 mM DTT (ThermoFisher

Scientific, Waltham, MA, USA; 18090050)

NEBNext End repair / dA-tailing Module (New England Biolabs, Ipswich, MA, USA; E7546)

NEB Blunt/TA Ligase Master Mix (New England Biolabs, Ipswich, MA, USA; M0367)

Set heating block to $65^{\circ} \mathrm{C}, 42^{\circ} \mathrm{C}, 37^{\circ} \mathrm{C}$

Save the programs listed below into a thermal cycler 


\section{A) Reverse transcription and strand-switching}

1. Prepare the following reaction:

\begin{tabular}{|ll|}
\hline Reagent & Volume \\
\hline 250-500 ng RNA & $\times \mu \mathrm{l}$ \\
VNP (10 pmol) & $2.5 \mu \mathrm{l}$ \\
-10 mM dNTPs & $1 \mu \mathrm{l}$ \\
RNase-free water & $\mathrm{x} \mu \mathrm{l}$ \\
\hline Total volume & $\mathbf{7 . 5} \boldsymbol{\mu l}$ \\
\hline
\end{tabular}

2. Mix gently by flicking the tube, and spin down.

3. Incubate at $65{ }^{\circ} \mathrm{C}$ for 5 minutes and then snap cool on a pre-chilled freezer block.

4. Add $2 \mu \mathrm{ISP}(10 \mathrm{pmol})$

5. Mix gently by flicking the tube, and spin down.

6. Incubate at $42{ }^{\circ} \mathrm{C}$ for 3 minutes.

7. Add $4 \mu$ l SuperScript ${ }^{\mathrm{TM}}$ IV VILO ${ }^{\mathrm{TM}}$ Master Mix and $6.5 \mu$ l nuclease free water

8. Mix gently by flicking the tube, and spin down.

9. Incubate using the following protocol:

\begin{tabular}{|llll|}
\hline Cycle step & Temperature & Time & No. of cycles \\
\hline Reverse transcription & $50^{\circ} \mathrm{C}$ & 15 mins & 1 \\
Strand switching & $42{ }^{\circ} \mathrm{C}$ & 10 mins & 1 \\
Heat inactivation & $80^{\circ} \mathrm{C}$ & 30 mins & 1 \\
\hline Hold & $4{ }^{\circ} \mathrm{C}$ & & \\
\hline
\end{tabular}

\section{B) RNA degradation and second strand synthesis}

1. Transfer the sample to a $1.5 \mathrm{ml}$ DNA LoBind Eppendorf tube.

2. Add $17 \mu \mathrm{l}$ of resuspended AMPure XP beads to the reaction and mix by flicking the tube.

3. Incubate on a Hula mixer (rotator mixer) for 5 minutes at RT.

4. Remove residue from lid by tapping the tube on the bench or spinning down slightly the sample

5. Pellet on a magnet.

6. Keep the tube on the magnet, and pipette off the supernatant. 
7. Keep on magnet, wash beads with $200 \mu \mathrm{l}$ of freshly prepared $70 \%$ ethanol without disturbing the pellet.

8. Remove the $70 \%$ ethanol using a pipette and discard.

9. Repeat.

10. Remove residue from lid by tapping the tube on the bench or spinning down slightly the sample.

11. Pipette off any residual $70 \%$ ethanol.

12. Briefly allow to dry for $10 \mathrm{~min}$.

13. Remove the tube from the magnetic rack and resuspend pellet in $20 \mu$ l Nucleasefree water.

14. Incubate on a Hula mixer (rotator mixer) for 10 minutes at RT.

15. Pellet beads on magnet until the eluate is clear and colourless.

16. Remove and retain $20 \mu \mathrm{l}$ of eluate into a clean $1.5 \mathrm{ml}$ Eppendorf DNA LoBind tube.

17.Prepare the following reaction in a $0.2 \mathrm{ml}$ thin-walled PCR tube:

\begin{tabular}{|lr|}
\hline Reagent & Volume \\
\hline 2x LongAmp Taq Master Mix & $25 \mu \mathrm{l}$ \\
PR2 Primer (PR2, 10 pmol) & $2 \mu \mathrm{l}$ \\
Reverse-transcribed sample from above & $20 \mu \mathrm{l}$ \\
Nuclease-free water & $3 \mu \mathrm{l}$ \\
\hline Total & $\mathbf{5 0} \boldsymbol{\mu l}$ \\
\hline
\end{tabular}

18. Incubate using the following protocol:

\begin{tabular}{|lrr|}
\hline Temperature & Time & No. of cycles \\
\hline $94{ }^{\circ} \mathrm{C}$ & $1 \mathrm{~min}$ & 1 \\
$50{ }^{\circ} \mathrm{C}$ & $1 \mathrm{~min}$ & 1 \\
$65^{\circ} \mathrm{C}$ & $15 \mathrm{~min}$ & 1 \\
\hline $4^{\circ} \mathrm{C}$ & $\infty$ & 1 \\
\hline
\end{tabular}

19. Transfer the sample into a clean $1.5 \mathrm{ml}$ Eppendorf DNA LoBind tube.

20. Add $40 \mu \mathrm{l}$ of resuspended AMPure XP beads to the reaction and mix by flicking the tube. 
21. Incubate on a Hula mixer (rotator mixer) for 5 minutes at RT.

22. Remove residue from lid by tapping the tube on the bench or spinning down slightly the sample

23. Pellet on a magnet.

24. Keep the tube on the magnet, and pipette off the supernatant.

25. Keep on magnet, wash beads with $200 \mu \mathrm{l}$ of freshly prepared $70 \%$ ethanol without disturbing the pellet.

26. Remove the $70 \%$ ethanol using a pipette and discard.

27. Repeat.

28. Remove residue from lid by tapping the tube on the bench or spinning down slightly the sample.

29. Pipette off any residual $70 \%$ ethanol.

30. Briefly allow to dry for $10 \mathrm{~min}$.

31. Remove the tube from the magnetic rack and resuspend pellet in $21 \mu \mathrm{l}$ Nucleasefree water.

32. Incubate on a Hula mixer (rotator mixer) for 10 minutes at RT

33. Pellet beads on magnet until the eluate is clear and colourless.

34. Remove and retain $21 \mu \mathrm{l}$ of eluate into a clean $1.5 \mathrm{ml}$ Eppendorf DNA LoBind tube.

35. Analyse $1 \mu \mathrm{l}$ of the strand-switched DNA for size, quantity and quality.

C) End-prep (NEBNext End repair / dA-tailing Module (New England Biolabs, Ipswich, MA, USA; E7546)

1. Perform end repair and dA-tailing of fragmented DNA as follows:

\begin{tabular}{|lr|}
\hline Reagent & Volume \\
\hline cDNA sample & $20 \mu \mathrm{l}$ \\
Nuclease-free water & $30 \mu \mathrm{l}$ \\
Ultra II End-prep reaction buffer & $7 \mu \mathrm{l}$ \\
Ultra II End-prep enzyme mix & $3 \mu \mathrm{l}$ \\
\hline Total & $\mathbf{6 0} \boldsymbol{\mu l}$ \\
\hline
\end{tabular}


2. Incubate using the following protocol:

\begin{tabular}{|llr|}
\hline Temperature & Time & No. of cycles \\
\hline $20^{\circ} \mathrm{C}$ & $5 \mathrm{~min}$ & 1 \\
$65{ }^{\circ} \mathrm{C}$ & $5 \mathrm{~min}$ & 1 \\
\hline
\end{tabular}

3. Transfer the sample into a clean $1.5 \mathrm{ml}$ Eppendorf DNA LoBind tube.

4. Add $60 \mu \mathrm{l}$ of resuspended AMPure XP beads to the reaction and mix by flicking the tube.

5. Incubate on a Hula mixer (rotator mixer) for 5 minutes at RT.

6. Remove residue from lid by tapping the tube on the bench or spinning down slightly the sample

7. Pellet on a magnet.

8. Keep the tube on the magnet, and pipette off the supernatant.

9. Keep on magnet, wash beads with $200 \mu \mathrm{l}$ of freshly prepared $70 \%$ ethanol without disturbing the pellet.

10. Remove the $70 \%$ ethanol using a pipette and discard.

11. Repeat.

12. Remove residue from lid by tapping the tube on the bench or spinning down slightly the sample.

13. Pipette off any residual $70 \%$ ethanol.

14. Briefly allow to dry for $10 \mathrm{~min}$.

15. Remove the tube from the magnetic rack and resuspend pellet in $30 \mu \mathrm{l}$ Nucleasefree water. Incubate for 2 min.

16. Pellet beads on magnet until the eluate is clear and colourless.

17. Remove and retain $30 \mu \mathrm{l}$ of eluate into a clean $1.5 \mathrm{ml}$ Eppendorf DNA LoBind tube. 


\section{D) Barcoding and Adapter Ligation}

1. Add the reagents in the order given below, mixing by flicking the tube between each sequential addition:

\begin{tabular}{|lr|}
\hline Reagent & Volume \\
\hline end-prepped cDNA sample & $22,5 \mu \mathrm{l}$ \\
Native Barcoding Kit 1D & $2.5 \mu \mathrm{l}$ \\
\hline Total & $\mathbf{2 5} \mathbf{~ \mathbf { l }}$ \\
\hline
\end{tabular}

2. Mix gently by flicking the tube and spin down

3. Incubate reaction for $10 \mathrm{~min}$ at room temperature.

4. Add $50 \mu$ of resuspended AMPure XP beads to the reaction and mix by flicking the tube.

5. Incubate on a Hula mixer (rotator mixer) for 5 minutes at RT.

6. Remove residue from lid by tapping the tube on the bench or spinning down slightly the sample

7. Pellet on a magnet.

8. Keep the tube on the magnet, and pipette off the supernatant.

9. Keep on magnet, wash beads with $200 \mu \mathrm{l}$ of freshly prepared $70 \%$ ethanol without disturbing the pellet.

10. Remove the $70 \%$ ethanol using a pipette and discard.

11. Repeat.

12. Remove residue from lid by tapping the tube on the bench or spinning down slightly the sample.

13. Pipette off any residual $70 \%$ ethanol.

14. Briefly allow to dry for $10 \mathrm{~min}$.

15. Remove the tube from the magnetic rack and resuspend pellet in $30 \mu \mathrm{l}$ Nucleasefree water. Incubate for $2 \mathrm{~min}$.

16. Pellet beads on magnet until the eluate is clear and colourless.

17. Remove and retain $26 \mu \mathrm{l}$ of eluate into a clean $1.5 \mathrm{ml}$ Eppendorf DNA LoBind tube.

18. Quantify $1 \mu$ l of eluted sample. 
19. Pool the barcoded samples at the desired ratio in a DNA LoBind $1.5 \mathrm{ml}$ Eppendorf tube. Aim for as high a concentration as possible which does not exceed $700 \mathrm{ng}$ total. If the total volume is $>30 \mu \mathrm{l}$, perform a $2.5 x$ AMPure clean up and elute in 30 $\mu \mathrm{l}$ of nuclease free water.

20. Dilute $700 \mathrm{ng}$ pooled sample to $50 \mu \mathrm{l}$ in Nuclease-free water.

21. Thaw and prepare the sequencing kit and NEB Blunt/TA Ligase Master Mix reagents as follows:

\begin{tabular}{|lr|}
\hline Reagent & Temperature \\
\hline ABB Buffer (ABB) & RT \\
Elution Buffer (ELB) & RT \\
Barcode Adapter Mix (BAM) & on ice \\
Running Buffer with Fuel Mix (RBF) & on ice \\
NEB Blunt/TA Ligase Master Mix & on ice \\
\hline
\end{tabular}

22. Take the pooled and barcoded DNA and perform adapter ligation as follows, mixing by flicking the tube between each sequential addition.

\begin{tabular}{|lr|}
\hline Reagent & Volume \\
\hline 700 ng pooled barcoded sample & $50 \mu \mathrm{l}$ \\
Barcode Adapter Mix (BAM) & $20 \mu \mathrm{l}$ \\
NEB Blunt/TA Ligase Master Mix & $25 \mu \mathrm{l}$ \\
\hline Total & $95 \mu \mathrm{l}$ \\
\hline
\end{tabular}

23. Mix gently by flicking the tube, and spin down.

24. Incubate the reaction for 10 minutes at RT.

25. Add $40 \mu \mathrm{l}$ of resuspended AMPure XP beads to the adapter ligation reaction from the previous step and mix by pipetting.

26. Incubate on a Hula mixer (rotator mixer) for 5 minutes at RT.

27. Place on magnetic rack, allow beads to pellet and pipette off supernatant.

28. Add $140 \mu \mathrm{l}$ of the ABB buffer to the beads. Close the tube lid, and resuspend the beads by flicking the tube.

29. Return the tube to the magnetic rack, allow beads to pellet and pipette off the supernatant.

30. Repeat.

31. Remove the tube from the magnetic rack and resuspend pellet in $12 \mu$ l Elution Buffer (ELB).

32. Pellet beads on magnet until the eluate is clear and colourless. 
33. Remove and retain $12 \mu \mathrm{l}$ of eluate into a clean $1.5 \mathrm{ml}$ Eppendorf DNA LoBind tube.

34. Dispose the pelleted beads

35. The prepared library is used for loading into the MinION Flow Cell. Store the library on ice until ready to load.

E) Priming the Flowcell R9.4

1. Before sequencing checklist.

2. Prepared library on ice.

3. Set up computer to run MinKNOW Hardware check complete.

4. Connect sequencing device to computer with SpotON Flow Cell inserted.

5. Set up Desktop Agent (if applicable) and complete Flow cell check.

6. Prepare the flow cell priming mix in a clean $1.5 \mathrm{ml}$ Eppendorf DNA LoBind tube:

\begin{tabular}{|ll|}
\hline Reagent & Volume \\
\hline RBF & $576 \mu \mathrm{l}$ \\
Nuclease-free water & $624 \mu \mathrm{l}$ \\
\hline Total & $\mathbf{1 2 0 0} \boldsymbol{\mu l}$ \\
\hline
\end{tabular}

7. Load $800 \mu \mathrm{l}$ of the priming mix into the flow cell via the priming port, avoiding the introduction of air bubbles. Wait for 5 minutes.

8. Prepare the library for loading as follows:

\begin{tabular}{|lr|}
\hline Reagent & Volume \\
\hline RBF & $35.0 \mu \mathrm{l}$ \\
\hline LLB & $25.5 \mu \mathrm{l}$ \\
\hline Nuclease-free water & $2.5 \mu \mathrm{l}$ \\
DNA library & $12 \mu \mathrm{l}$ \\
\hline Total & $\mathbf{7 5 \mu \mathrm { l }}$ \\
\hline
\end{tabular}

9. Complete the flow cell priming:

10. Gently lift the SpotON sample port cover to make the SpotON sample port accessible.

11. Load $200 \mu \mathrm{l}$ of the priming mix into the flow cell via the priming port (not the SpotON sample port), avoiding the introduction of air bubbles.

12. Mix the prepared library gently by pipetting up and down just prior to loading. 
13. Add of $75 \mu \mathrm{l}$ sample to the flow cell via the SpotON sample port in a dropwise fashion.

14. Ensure each drop flows into the port before adding the next.

15. Gently replace the SpotON sample port cover, making sure the bung enters the SpotON port, close the priming port and replace the MinION lid.

F) Start the sequencing run 
Additional file 2: List of sequences in the offline database for FMDV serotyping

Data Base Accession,Serotype

P1
DQ009742,FMDV-SAT3

DQ009741,FMDV-SAT3

DQ009740,FMDV-SAT3

DQ009739,FMDV-SAT3

KJ999944,FMDV-SAT2

KJ999943,FMDV-SAT2

KJ999942,FMDV-SAT2

KJ999941,FMDV-SAT2

KJ999940,FMDV-SAT2

KJ999939,FMDV-SAT2

KJ999938,FMDV-SAT2

KJ999937,FMDV-SAT2

KJ999936,FMDV-SAT2

KJ999935,FMDV-SAT2

KJ999934,FMDV-SAT2

KJ999933,FMDV-SAT2

KJ999932,FMDV-SAT2

JQ639296,FMDV-SAT2

JQ639295,FMDV-SAT2

JQ639294,FMDV-SAT2

JQ639293,FMDV-SAT2

JQ639292,FMDV-SAT2

JQ639291,FMDV-SAT2

JQ639290,FMDV-SAT2

JQ639289,FMDV-SAT2

GU194494,FMDV-SAT2

GU194493,FMDV-SAT2

GU194492,FMDV-SAT2

GU194491,FMDV-SAT2

GU194490,FMDV-SAT2

GU194489,FMDV-SAT2

GU194488,FMDV-SAT2

DQ009738,FMDV-SAT2

DQ009737,FMDV-SAT2

DQ009736,FMDV-SAT2

DQ009735,FMDV-SAT2

DQ009734,FMDV-SAT2

DQ009733,FMDV-SAT2

DQ009732,FMDV-SAT2

DQ009731,FMDV-SAT2

DQ009730,FMDV-SAT2

DQ009729,FMDV-SAT2

DQ009728,FMDV-SAT2

DQ009727,FMDV-SAT2

DQ009726,FMDV-SAT2
KJ999931,FMDV-SAT1

KJ999930,FMDV-SAT1

KJ999929,FMDV-SAT1

KJ999928,FMDV-SAT1

KJ999927,FMDV-SAT1

KJ999926,FMDV-SAT1

KJ999925,FMDV-SAT1

KJ999924,FMDV-SAT1

KJ999923,FMDV-SAT1

KJ999922,FMDV-SAT1

KJ999921,FMDV-SAT1

KJ999920,FMDV-SAT1

KJ999919,FMDV-SAT1

KJ999918,FMDV-SAT1

KJ999917,FMDV-SAT1

KJ999916,FMDV-SAT1

KJ999915,FMDV-SAT1

KJ999914,FMDV-SAT1

KJ999913,FMDV-SAT1

KJ999912,FMDV-SAT1

KJ999911,FMDV-SAT1

KJ999910,FMDV-SAT1

KJ999909,FMDV-SAT1

KJ999908,FMDV-SAT1

JQ692596,FMDV-SAT1

JQ692595,FMDV-SAT1

GU194503,FMDV-SAT1

GU194502,FMDV-SAT1

GU194501,FMDV-SAT1

GU194500,FMDV-SAT1

GU194499,FMDV-SAT1

GU194498,FMDV-SAT1

GU194497,FMDV-SAT1

GU194496,FMDV-SAT1

GU194495,FMDV-SAT1

DQ009725,FMDV-SAT1

DQ009724,FMDV-SAT1

DQ009723,FMDV-SAT1

DQ009722,FMDV-SAT1

DQ009721,FMDV-SAT1

DQ009720,FMDV-SAT1

DQ009719,FMDV-SAT1

DQ009718,FMDV-SAT1

DQ009717,FMDV-SAT1

DQ009716,FMDV-SAT1

DQ009715,FMDV-SAT1 


\begin{tabular}{|c|c|}
\hline AY770519,FMDV-SAT1 & KP202877,FMDV-O \\
\hline AF283429,FMDV-SAT1 & KJ831747,FMDV-O \\
\hline KU530146,FMDV-O & KJ831746,FMDV-O \\
\hline KU365846,FMDV-O & KJ831745,FMDV-O \\
\hline KU365845,FMDV-O & KJ831744,FMDV-O \\
\hline KU365844,FMDV-O & KJ831743,FMDV-O \\
\hline KR149728,FMDV-O & KJ831742,FMDV-O \\
\hline KR149727,FMDV-O & KJ831741,FMDV-O \\
\hline KR149726,FMDV-O & KJ831740,FMDV-O \\
\hline KR149725,FMDV-O & KJ831739,FMDV-O \\
\hline KR149724,FMDV-O & KJ831738,FMDV-O \\
\hline KR149723,FMDV-O & KJ831737,FMDV-O \\
\hline KR149722,FMDV-O & KJ831736,FMDV-O \\
\hline KR149721,FMDV-O & KJ831735,FMDV-O \\
\hline KR149720,FMDV-O & KJ831734,FMDV-O \\
\hline KR149719,FMDV-O & KJ831733,FMDV-O \\
\hline KR149718,FMDV-O & KJ831732,FMDV-O \\
\hline KR149717,FMDV-O & KJ831731,FMDV-O \\
\hline KR149716,FMDV-O & KJ831730,FMDV-O \\
\hline KR149715,FMDV-O & KJ831729,FMDV-O \\
\hline KR149714,FMDV-O & KJ831728,FMDV-O \\
\hline KR149713,FMDV-O & KJ831727,FMDV-O \\
\hline KR149712,FMDV-O & KJ831726,FMDV-O \\
\hline KR149711,FMDV-O & KJ831725,FMDV-O \\
\hline KR149710,FMDV-O & KJ831724,FMDV-O \\
\hline KR149709,FMDV-O & KJ831723,FMDV-O \\
\hline KR149708,FMDV-O & KJ831722,FMDV-O \\
\hline KR149707,FMDV-O & KJ831721,FMDV-O \\
\hline KR149706,FMDV-O & KJ831720,FMDV-O \\
\hline KR149705,FMDV-O & KJ831719,FMDV-O \\
\hline KR149704,FMDV-O & KJ831718,FMDV-O \\
\hline KR149703,FMDV-O & KJ831717,FMDV-O \\
\hline KR149702,FMDV-O & KJ831714,FMDV-O \\
\hline KR149701,FMDV-O & KJ831713,FMDV-O \\
\hline KR149700,FMDV-O & KJ831712,FMDV-O \\
\hline KR149699,FMDV-O & KJ831711,FMDV-O \\
\hline KR149698,FMDV-O & KJ831710,FMDV-O \\
\hline KR149697,FMDV-O & KJ831709,FMDV-O \\
\hline KP835578,FMDV-O & KJ831708,FMDV-O \\
\hline KP822947,FMDV-O & KJ831707,FMDV-O \\
\hline KP822946,FMDV-O & KJ831706,FMDV-O \\
\hline KP822945,FMDV-O & KJ831705,FMDV-O \\
\hline KP822942,FMDV-O & KJ831704,FMDV-O \\
\hline KP720595,FMDV-O & KJ831703,FMDV-O \\
\hline KP720594,FMDV-O & KJ831702,FMDV-O \\
\hline KP202878,FMDV-O & KJ831701,FMDV-O \\
\hline
\end{tabular}




\begin{tabular}{|c|c|}
\hline KJ831700,FMDV-O & AF292107,FMDV-O \\
\hline KJ831699,FMDV-O & M90372,FMDV-C \\
\hline KJ831698,FMDV-O & M90381,FMDV-C \\
\hline KJ831697,FMDV-O & M90376,FMDV-C \\
\hline KJ831696,FMDV-O & M90367,FMDV-C \\
\hline KJ831695,FMDV-O & M90368,FMDV-C \\
\hline KJ831694,FMDV-O & L29061,FMDV-C \\
\hline KJ831693,FMDV-O & L29062,FMDV-C \\
\hline KJ831692,FMDV-O & KU380332,FMDV-Asia1 \\
\hline KJ831691,FMDV-O & KU365794,FMDV-Asia1 \\
\hline KJ831690,FMDV-O & KU365793,FMDV-Asia1 \\
\hline KJ831689,FMDV-O & KU365792,FMDV-Asia1 \\
\hline KJ831688,FMDV-O & KU365791,FMDV-Asia1 \\
\hline KJ831687,FMDV-O & KU365790,FMDV-Asia1 \\
\hline KJ831686,FMDV-O & KU365789,FMDV-Asia1 \\
\hline KJ831684,FMDV-O & KU365788,FMDV-Asia1 \\
\hline KJ831683,FMDV-O & KU365787,FMDV-Asia1 \\
\hline KJ831682,FMDV-O & KU365786,FMDV-Asia1 \\
\hline KJ831681,FMDV-O & KU365785,FMDV-Asia1 \\
\hline KJ831680,FMDV-O & KU365784,FMDV-Asia1 \\
\hline KJ831679,FMDV-O & KU365783,FMDV-Asia1 \\
\hline KJ831678,FMDV-O & KU365782,FMDV-Asia1 \\
\hline KJ831677,FMDV-O & JQ818553,FMDV-Asia1 \\
\hline KJ831676,FMDV-O & AF292106,FMDV-Asia1 \\
\hline KJ831675,FMDV-O & KU737534,FMDV-A \\
\hline KJ831674,FMDV-O & KU737533,FMDV-A \\
\hline KJ831671,FMDV-O & KU737532,FMDV-A \\
\hline KJ831670,FMDV-O & KU737531,FMDV-A \\
\hline KJ831669,FMDV-O & KU737530,FMDV-A \\
\hline KJ831668,FMDV-O & KU737529,FMDV-A \\
\hline KJ831667,FMDV-O & KU737528,FMDV-A \\
\hline KJ831666,FMDV-O & KU737527,FMDV-A \\
\hline KJ831665,FMDV-O & KU612884,FMDV-A \\
\hline KJ831664,FMDV-O & KU612883,FMDV-A \\
\hline KJ831663,FMDV-O & KU612882,FMDV-A \\
\hline KJ606984,FMDV-O & KU612881,FMDV-A \\
\hline KJ606983,FMDV-O & KU612880,FMDV-A \\
\hline KJ606982,FMDV-O & KU612879,FMDV-A \\
\hline KJ606981,FMDV-O & KU612878,FMDV-A \\
\hline KJ606980,FMDV-O & KU612877,FMDV-A \\
\hline KJ606979,FMDV-O & KU612876,FMDV-A \\
\hline KJ606978,FMDV-O & KU612875,FMDV-A \\
\hline KJ606977,FMDV-O & KU612874,FMDV-A \\
\hline JQ818555,FMDV-O & KU612873,FMDV-A \\
\hline HQ663879,FMDV-O & KU612872,FMDV-A \\
\hline U82271,FMDV-O & KU612871,FMDV-A \\
\hline
\end{tabular}




\begin{tabular}{|c|c|}
\hline KU612870,FMDV-A & KU612824,FMDV-A \\
\hline KU612869,FMDV-A & KU612823,FMDV-A \\
\hline KU612868,FMDV-A & KU612822,FMDV-A \\
\hline KU612867,FMDV-A & KU612821,FMDV-A \\
\hline KU612866,FMDV-A & KU612820,FMDV-A \\
\hline KU612865,FMDV-A & KU612818,FMDV-A \\
\hline KU612864,FMDV-A & KU612817,FMDV-A \\
\hline KU612863,FMDV-A & KU612816,FMDV-A \\
\hline KU612862,FMDV-A & KU291465,FMDV-A \\
\hline KU612861,FMDV-A & KU291464,FMDV-A \\
\hline KU612860,FMDV-A & KU291463,FMDV-A \\
\hline KU612859,FMDV-A & KU291462,FMDV-A \\
\hline KU612858,FMDV-A & KU291461,FMDV-A \\
\hline KU612857,FMDV-A & KU291460,FMDV-A \\
\hline KU612856,FMDV-A & KU208000,FMDV-A \\
\hline KU612855,FMDV-A & KR188514,FMDV-A \\
\hline KU612854,FMDV-A & KR188513,FMDV-A \\
\hline KU612853,FMDV-A & KM189191,FMDV-A \\
\hline KU612852,FMDV-A & KM189190,FMDV-A \\
\hline KU612851,FMDV-A & KM189189,FMDV-A \\
\hline KU612850,FMDV-A & KM189188,FMDV-A \\
\hline KU612849,FMDV-A & KM189187,FMDV-A \\
\hline KU612848,FMDV-A & KM189186,FMDV-A \\
\hline KU612847,FMDV-A & KM189185,FMDV-A \\
\hline KU612846,FMDV-A & KM189184,FMDV-A \\
\hline KU612845,FMDV-A & KM189183,FMDV-A \\
\hline KU612844,FMDV-A & KJ146961,FMDV-A \\
\hline KU612843,FMDV-A & KJ146960,FMDV-A \\
\hline KU612842,FMDV-A & KJ146959,FMDV-A \\
\hline KU612841,FMDV-A & KJ146958,FMDV-A \\
\hline KU612840,FMDV-A & KJ146957,FMDV-A \\
\hline KU612839,FMDV-A & KJ146956,FMDV-A \\
\hline KU612838,FMDV-A & KJ146955,FMDV-A \\
\hline KU612837,FMDV-A & KJ146954,FMDV-A \\
\hline KU612836,FMDV-A & KJ146953,FMDV-A \\
\hline KU612835,FMDV-A & KJ146952,FMDV-A \\
\hline KU612834,FMDV-A & KJ146951,FMDV-A \\
\hline KU612833,FMDV-A & KJ146950,FMDV-A \\
\hline KU612832,FMDV-A & KJ146949,FMDV-A \\
\hline KU612831,FMDV-A & KJ146948,FMDV-A \\
\hline KU612830,FMDV-A & KJ146947,FMDV-A \\
\hline KU612829,FMDV-A & KJ146946,FMDV-A \\
\hline KU612828,FMDV-A & KJ146945,FMDV-A \\
\hline KU612827,FMDV-A & KJ146944,FMDV-A \\
\hline KU612826,FMDV-A & KJ146943,FMDV-A \\
\hline KU612825,FMDV-A & KJ146942,FMDV-A \\
\hline
\end{tabular}




\begin{tabular}{|c|c|}
\hline KJ146941,FMDV-A & KJ146895,FMDV-A \\
\hline KJ146940,FMDV-A & KJ146894,FMDV-A \\
\hline KJ146939,FMDV-A & KJ146893,FMDV-A \\
\hline KJ146938,FMDV-A & KJ146892,FMDV-A \\
\hline KJ146937,FMDV-A & KJ146891,FMDV-A \\
\hline KJ146936,FMDV-A & KJ146890,FMDV-A \\
\hline KJ146935,FMDV-A & KJ146889,FMDV-A \\
\hline KJ146934,FMDV-A & KJ146888,FMDV-A \\
\hline KJ146933,FMDV-A & KJ146887,FMDV-A \\
\hline KJ146932,FMDV-A & KJ146886,FMDV-A \\
\hline KJ146931,FMDV-A & KJ146885,FMDV-A \\
\hline KJ146930,FMDV-A & KJ146884,FMDV-A \\
\hline KJ146929,FMDV-A & KJ146883,FMDV-A \\
\hline KJ146928,FMDV-A & KJ146882,FMDV-A \\
\hline KJ146927,FMDV-A & KJ146881,FMDV-A \\
\hline KJ146926,FMDV-A & KJ146880,FMDV-A \\
\hline KJ146925,FMDV-A & KJ146879,FMDV-A \\
\hline KJ146924,FMDV-A & KJ146878,FMDV-A \\
\hline KJ146923,FMDV-A & KJ146877,FMDV-A \\
\hline KJ146922,FMDV-A & KJ146876,FMDV-A \\
\hline KJ146921,FMDV-A & KJ146875,FMDV-A \\
\hline KJ146920,FMDV-A & KJ146874,FMDV-A \\
\hline KJ146919,FMDV-A & KJ146873,FMDV-A \\
\hline KJ146918,FMDV-A & KJ146872,FMDV-A \\
\hline KJ146917,FMDV-A & KJ146871,FMDV-A \\
\hline KJ146916,FMDV-A & KJ146870,FMDV-A \\
\hline KJ146915,FMDV-A & KJ146869,FMDV-A \\
\hline KJ146914,FMDV-A & KJ146868,FMDV-A \\
\hline KJ146913,FMDV-A & KJ146867,FMDV-A \\
\hline KJ146912,FMDV-A & KF152935,FMDV-A \\
\hline KJ146911,FMDV-A & JQ818554,FMDV-A \\
\hline KJ146910,FMDV-A & HQ666887,FMDV-A \\
\hline KJ146909,FMDV-A & HQ666886,FMDV-A \\
\hline KJ146908,FMDV-A & HQ666885,FMDV-A \\
\hline KJ146907,FMDV-A & HQ666884,FMDV-A \\
\hline KJ146906,FMDV-A & HQ666883,FMDV-A \\
\hline KJ146905,FMDV-A & HQ666882,FMDV-A \\
\hline KJ146904,FMDV-A & HQ666881,FMDV-A \\
\hline KJ146903,FMDV-A & HQ666880,FMDV-A \\
\hline KJ146902,FMDV-A & HQ666879,FMDV-A \\
\hline KJ146901,FMDV-A & HQ666878,FMDV-A \\
\hline KJ146900,FMDV-A & HQ666877,FMDV-A \\
\hline KJ146899,FMDV-A & HQ666876,FMDV-A \\
\hline KJ146898,FMDV-A & HQ666875,FMDV-A \\
\hline KJ146897,FMDV-A & HQ666874,FMDV-A \\
\hline KJ146896,FMDV-A & HQ666873,FMDV-A \\
\hline
\end{tabular}


Data Base Accession,Serotype

full genome AY593771,FMDV-A

AY593772,FMDV-A

AY593773,FMDV-A

AY593775,FMDV-A

AY593782,FMDV-A

AY593783,FMDV-A

AY593784,FMDV-A

AY593785,FMDV-A

AY593786,FMDV-A

AY593787,FMDV-A

AY593788,FMDV-A

AY593789,FMDV-A

AY593790,FMDV-A

AY593791,FMDV-A

AY593792,FMDV-A

AY593793,FMDV-A

AY593794,FMDV-A

AY593801,FMDV-A

AY593802,FMDV-A

AY593803,FMDV-A

AY304994,FMDV-Asia1

AY390432,FMDV-Asia1

AY593795,FMDV-Asia1

AY593796,FMDV-Asia1

AY593797,FMDV-Asia1

AY593798,FMDV-Asia1

AY593799,FMDV-Asia1

AY593800,FMDV-Asia1

AY687334,FMDV-Asia1

DQ533483,FMDV-Asia1

DQ989319,FMDV-Asia1

DQ989321,FMDV-Asia1

EF149009,FMDV-Asia1

EF149010,FMDV-Asia1

EF614458,FMDV-Asia1

FJ906802,FMDV-Asia1

HQ632774,FMDV-Asia1

JF739177,FMDV-Asia1

KM268898,FMDV-Asia1

KR073010,FMDV-Asia1

KY446901,FMDV-Asia1

AF274010,FMDV-C

AM409325,FMDV-C

AY593810,FMDV-C

DQ409183,FMDV-C
DQ409184,FMDV-C

DQ409185,FMDV-C

DQ409186,FMDV-C

DQ409187,FMDV-C

DQ409188,FMDV-C

DQ409189,FMDV-C

DQ409190,FMDV-C

DQ409191,FMDV-C

FJ824812,FMDV-C

AY593804,FMDV-C

AY593805,FMDV-C

AY593806,FMDV-C

AY593807,FMDV-C

AY593808,FMDV-C

AY593809,FMDV-C

KM268897,FMDV-C

AF026168,FMDV-O

AF506822,FMDV-O

AJ633821,FMDV-O

AY333431,FMDV-O

AY359854,FMDV-O

AY593811,FMDV-O

AY593812,FMDV-O

AY593813,FMDV-O

AY593826,FMDV-O

AY593827,FMDV-O

AY593828,FMDV-O

AY593829,FMDV-O

AY593830,FMDV-O

AY593831,FMDV-O

AY593832,FMDV-O

AY593833,FMDV-O

AY593834,FMDV-O

AY593835,FMDV-O

AY593836,FMDV-O

AY593837,FMDV-O

AY593838,FMDV-SAT1

AY593839,FMDV-SAT1

AY593840,FMDV-SAT1

AY593841,FMDV-SAT1

AY593842,FMDV-SAT1

AY593843,FMDV-SAT1

AY593844,FMDV-SAT1

AY593845,FMDV-SAT1

AY593846,FMDV-SAT1

JF749860,FMDV-SAT1 
KM268899,FMDV-SAT1

KU821590,FMDV-SAT1

MF678823,FMDV-SAT1

MF678824,FMDV-SAT1

MF678825,FMDV-SAT1

MF678826,FMDV-SAT1

AF540910,FMDV-SAT2

AY593847,FMDV-SAT2

AY593848,FMDV-SAT2

AY593849,FMDV-SAT2

JF749862,FMDV-SAT2

JX014255,FMDV-SAT2

JX014256,FMDV-SAT2

KC440884,FMDV-SAT2

KM268900,FMDV-SAT2

KU821592,FMDV-SAT2

AY593850,FMDV-SAT3

AY593851,FMDV-SAT3

AY593852,FMDV-SAT3

AY593853,FMDV-SAT3

KJ820999,FMDV-SAT3

KM268901,FMDV-SAT3

KX375417,FMDV-SAT3 


\section{Data analysis Step I}

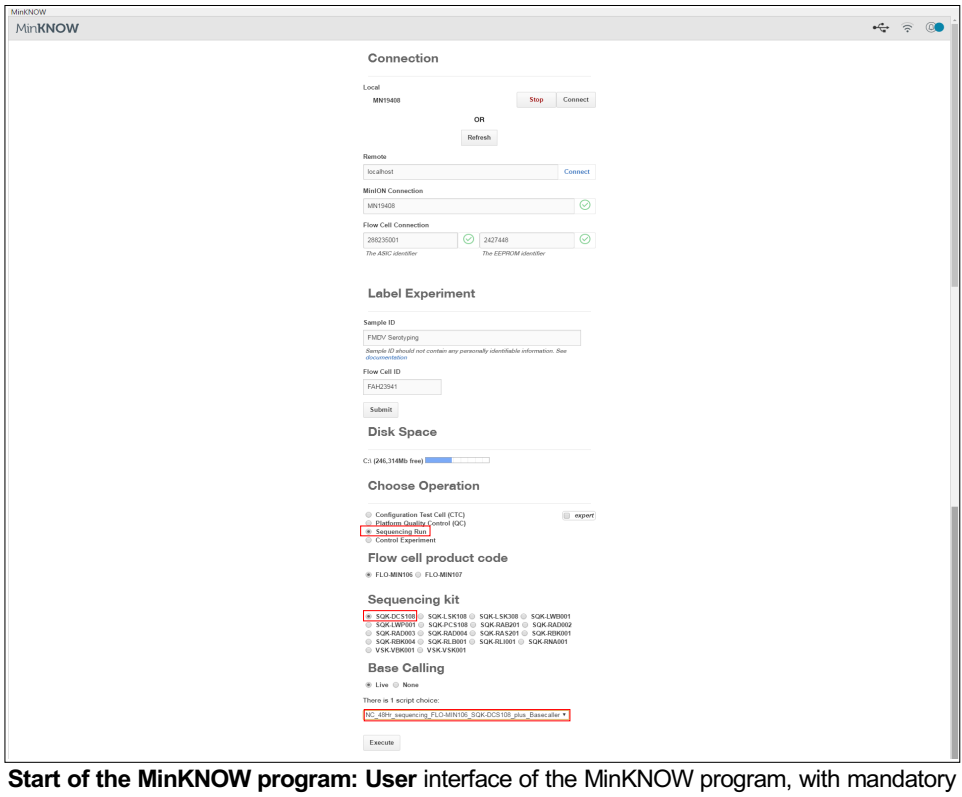

\section{Data analysis Step II}

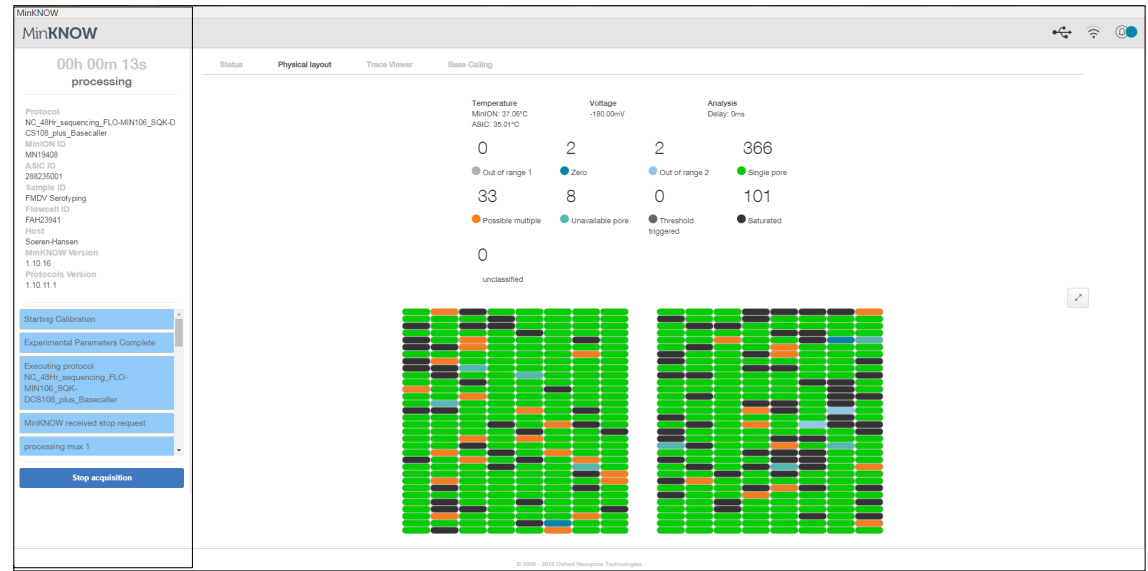

Sequencing run: MinKNOW interface for monitoring of sequencing run. Green dots show active nanopores for sequencing. 


\section{Data analysis Step III}

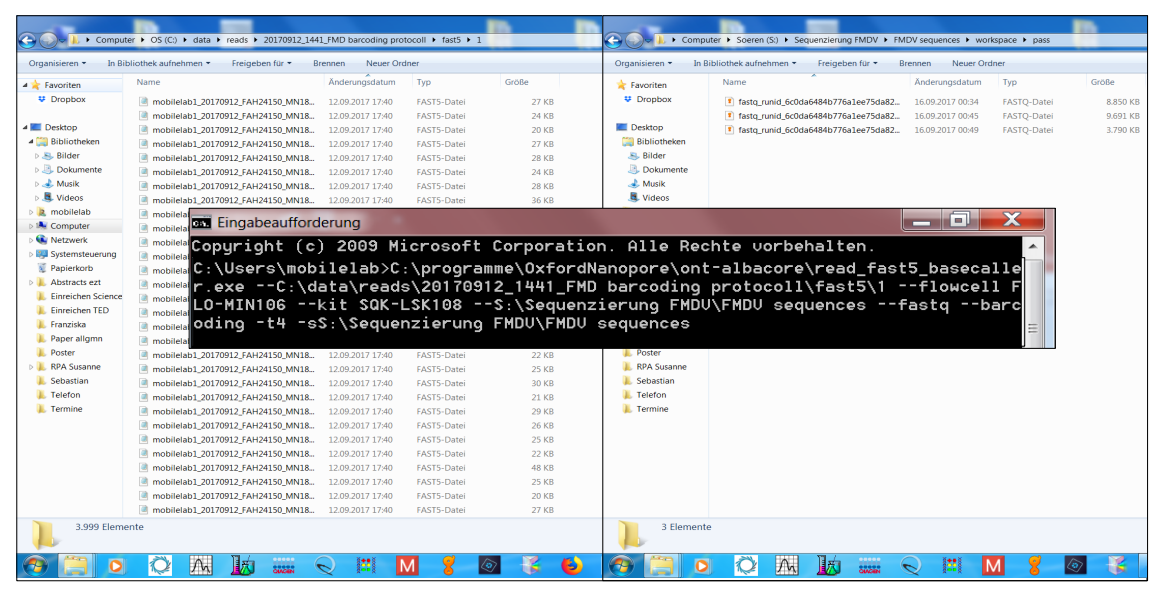

Processing sequencing files: from Fast5 to FASTA reads using ALBACORE.

\section{Data analysis Step IV}

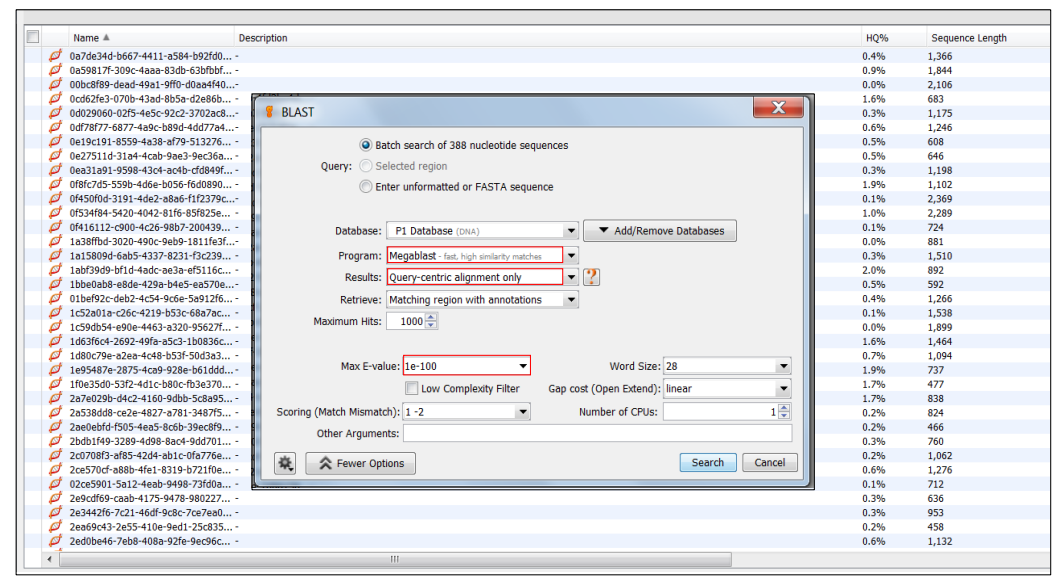

BLAST search: Reads yielded from Barcode 1 sequencing files. In red frame, settings for MEGABLAST algorithm in Geneious. 


\section{Data analysis Step V}

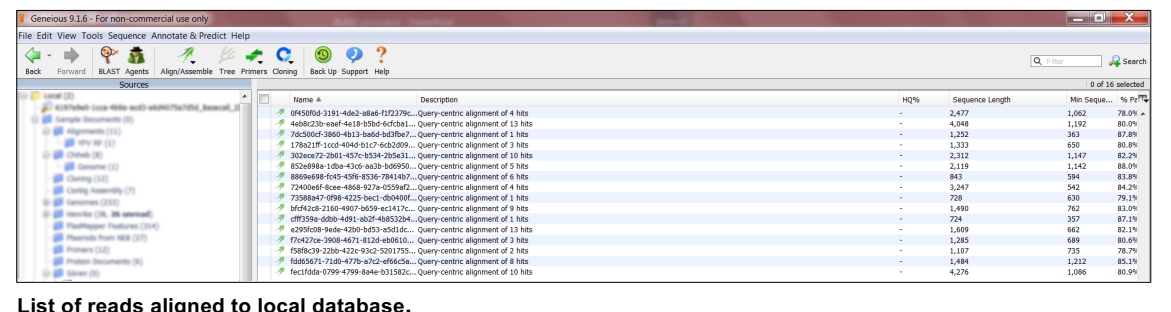

List of reads aligned to local database.

\section{Data analysis Step VI}

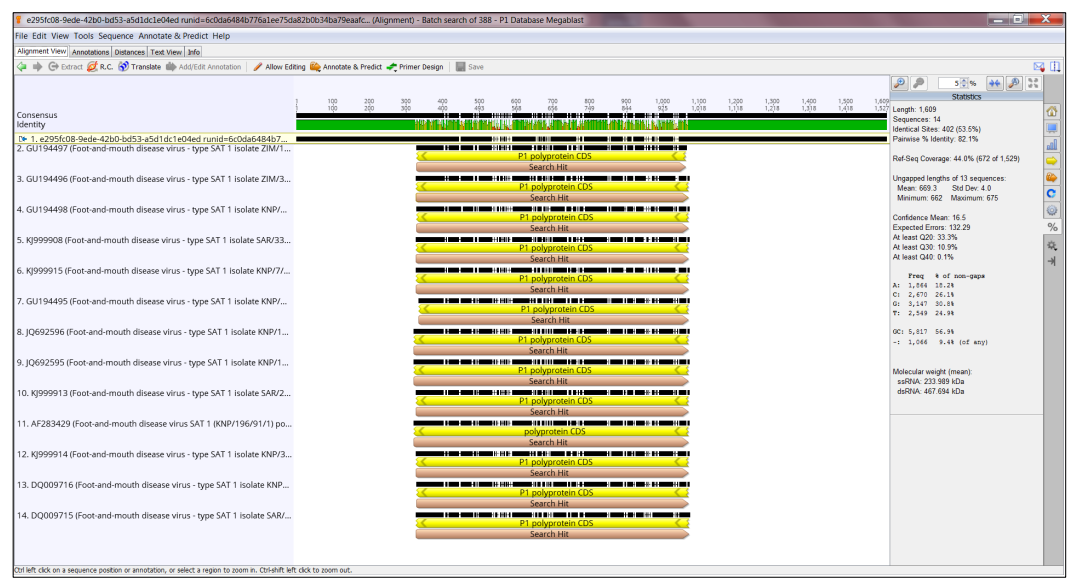

View on Query-centric alignment of sequences from local database to a single read. 


\section{Data analysis Step VII}

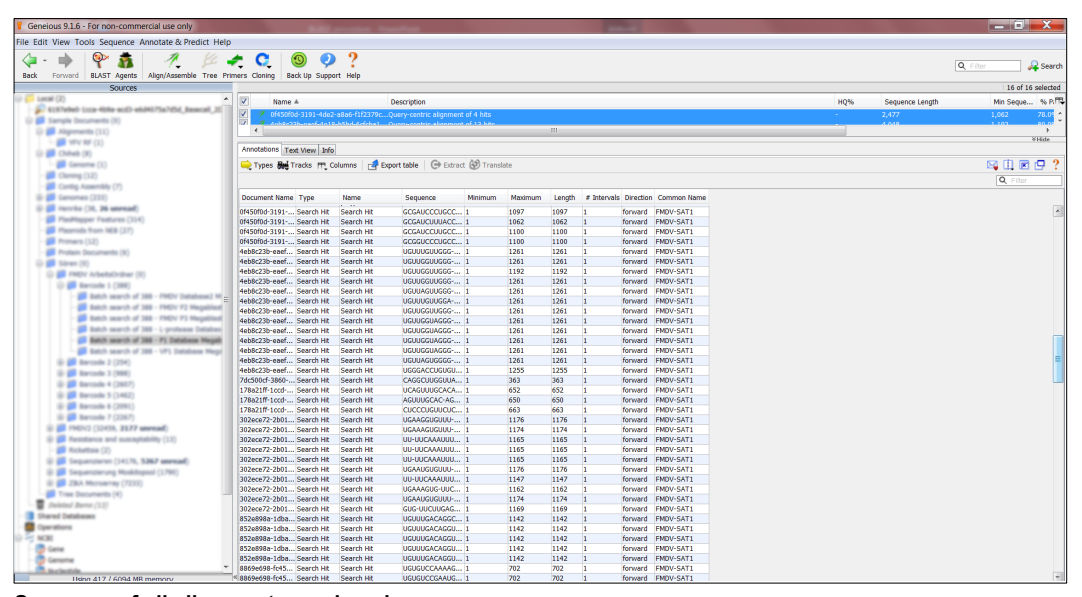

Summary of all alignments produced. 


\title{
Supplementary Material
}

\section{Diagnosing Zika virus infection against a background of other flaviviruses:}

\section{Studies in high resolution serological analysis}

\author{
Sören Hansen ${ }^{1 \#}$, Sven-Kevin Hotop ${ }^{2 \& 3 \#}$, Oumar Faye ${ }^{4}$, Oumar Ndiaye ${ }^{4}$, Susanne \\ Böhlken-Fascher ${ }^{1}$, Rodrigo Pessôa ${ }^{5}$, Frank Hufert ${ }^{6}$, Christiane Stahl-Hennig ${ }^{7}$, Ronald \\ Frank $^{8}$, Claus-Peter Czerny ${ }^{1}$, Jonas Schmidt-Chanasit ${ }^{9 \& 10}$, Sabri S. Sanabani ${ }^{5}$, \\ Amadou A. Sall ${ }^{4}$, Matthias Niedrig ${ }^{11}$, Mark Brönstrup ${ }^{2 \& 3}$, Hans-Joachim Fritz ${ }^{12 \pi}$, Ahmed
} Abd El Wahed ${ }^{1 \pi^{*}}$

1) Division of Microbiology and Animal Hygiene, University of Goettingen, 37075, Germany

2) Department of Chemical Biology, Helmholtz-Zentrum für Infektionsforschung, Inhoffenstrasse 7, 38124 Braunschweig,

Germany

3) Deutsches Zentrum für Infektionsforschung (DZIF), Standort Hannover-Braunschweig, Germany

4) Institut Pasteur de Dakar, Dakar, Senegal

5) Laboratory of Dermatology and Immunodeficiencies, LIM-56, Department of Dermatology, Tropical Medicine Institute of São Paulo, University of São Paulo, São Paulo, Brazil

6) Institute of Microbiology and Virology, Brandenburg Medical School Fontane, Senftenberg, Germany

7) Deutsches Primatenzentrum GmbH, Leibniz-Institut für Primatenforschung, Unit of Infection Models, Göttingen, Germany

8) AIMS Scientific Products GmbH, Berlin, Germany

9) Bernhard Nocht Institute for Tropical Medicine, WHO Collaborating Centre for Arbovirus and Hemorrhagic Fever Reference and Research, Hamburg, Germany

10) German Centre for Infection Research (DZIF), partner site Hamburg-Luebeck-Borstel, Hamburg, Germany

11) Robert Koch Institut, Nordufer 20, 13353 Berlin, Germany

12) Akademie der Wissenschaften zu Göttingen, Theaterstraße 7, 37073 Göttingen, Germany

\#Equal contribution

"Joint senior authors

*Corresponding author 
Table S1: Experimentally identified ATRs: Comprehensive List.
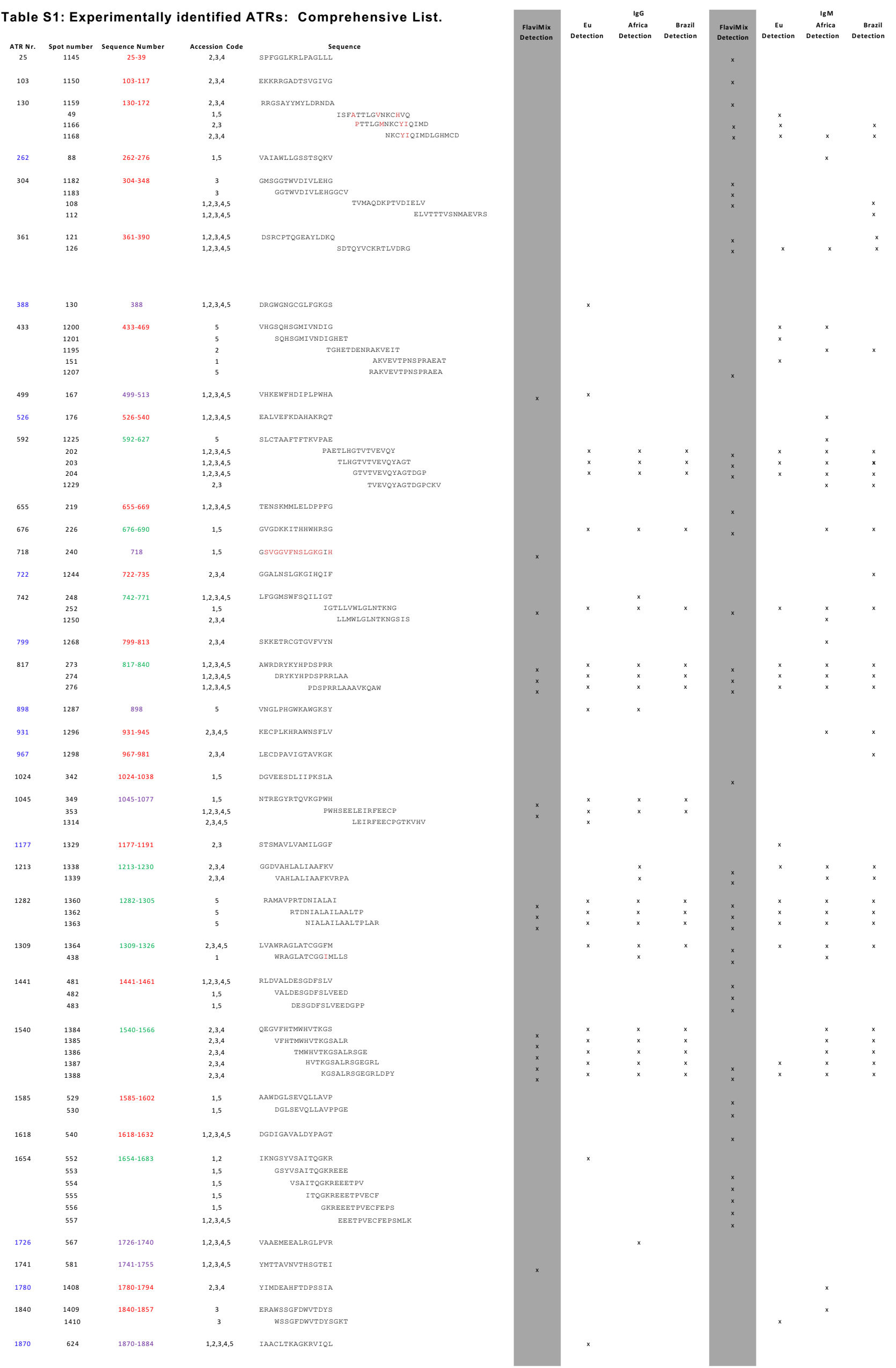


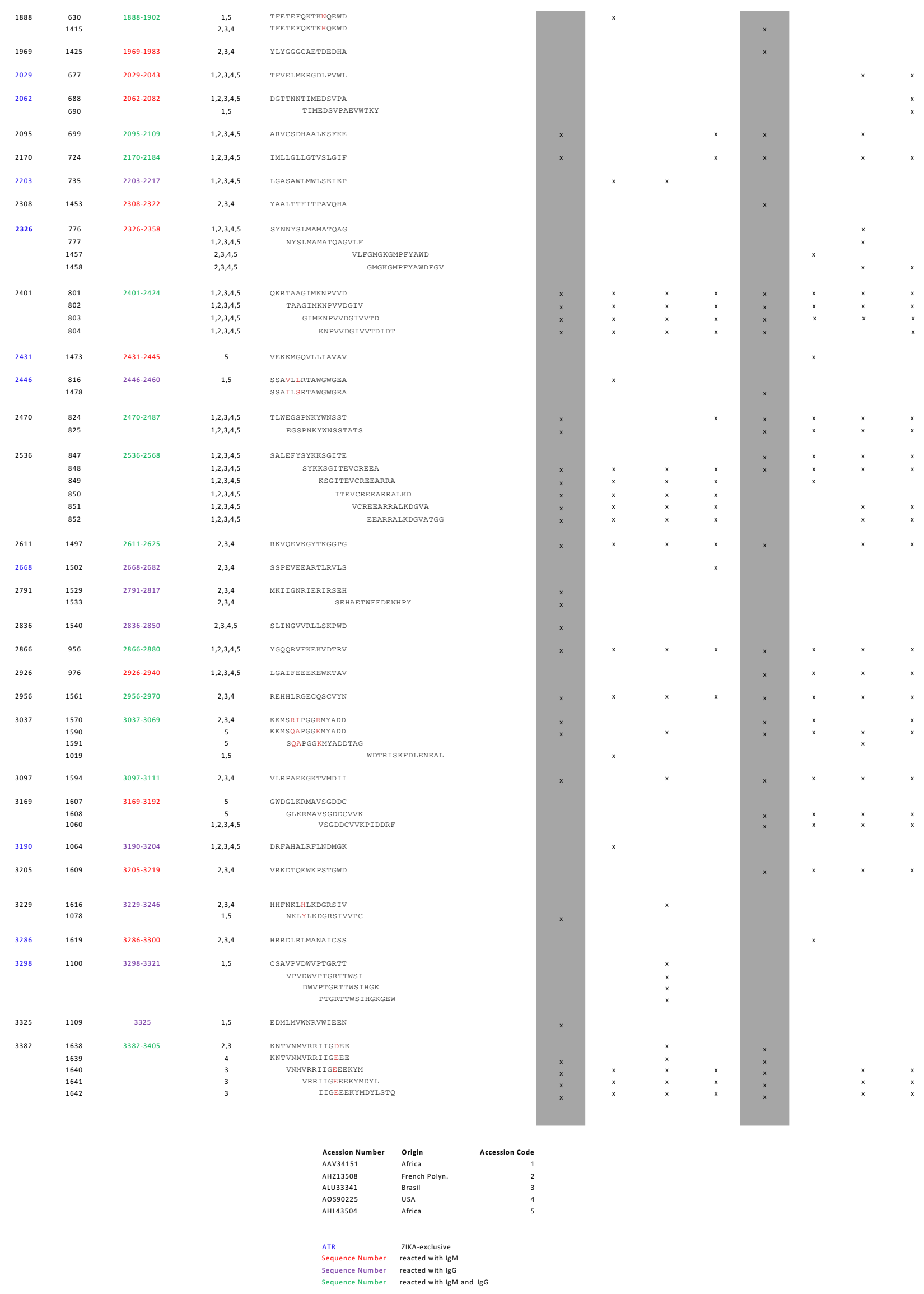



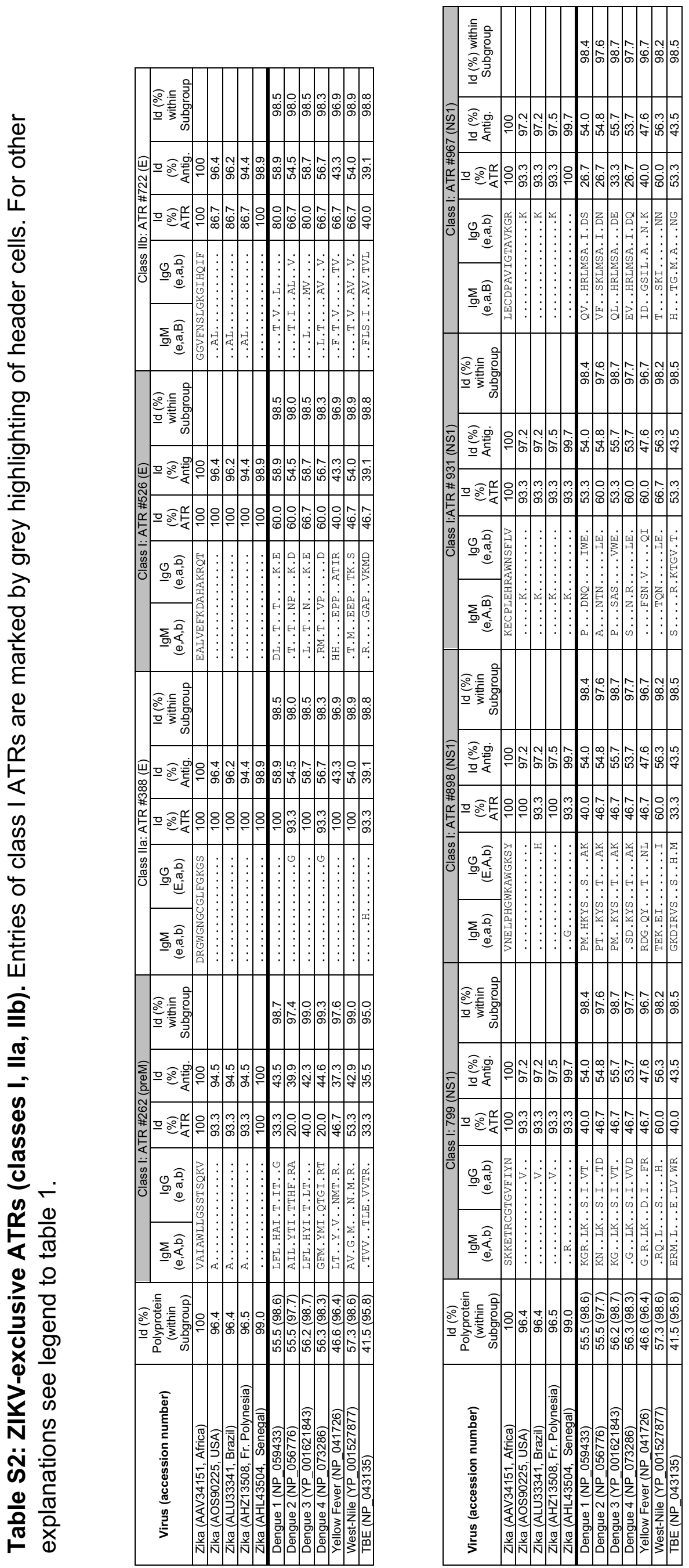


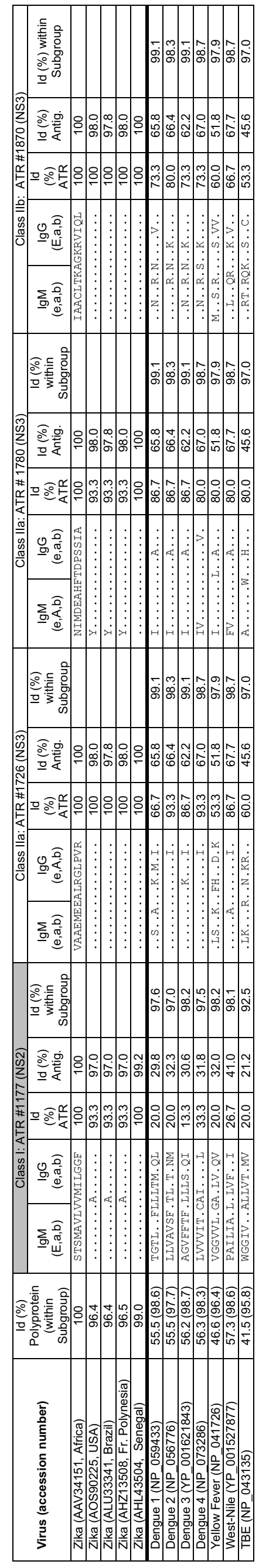
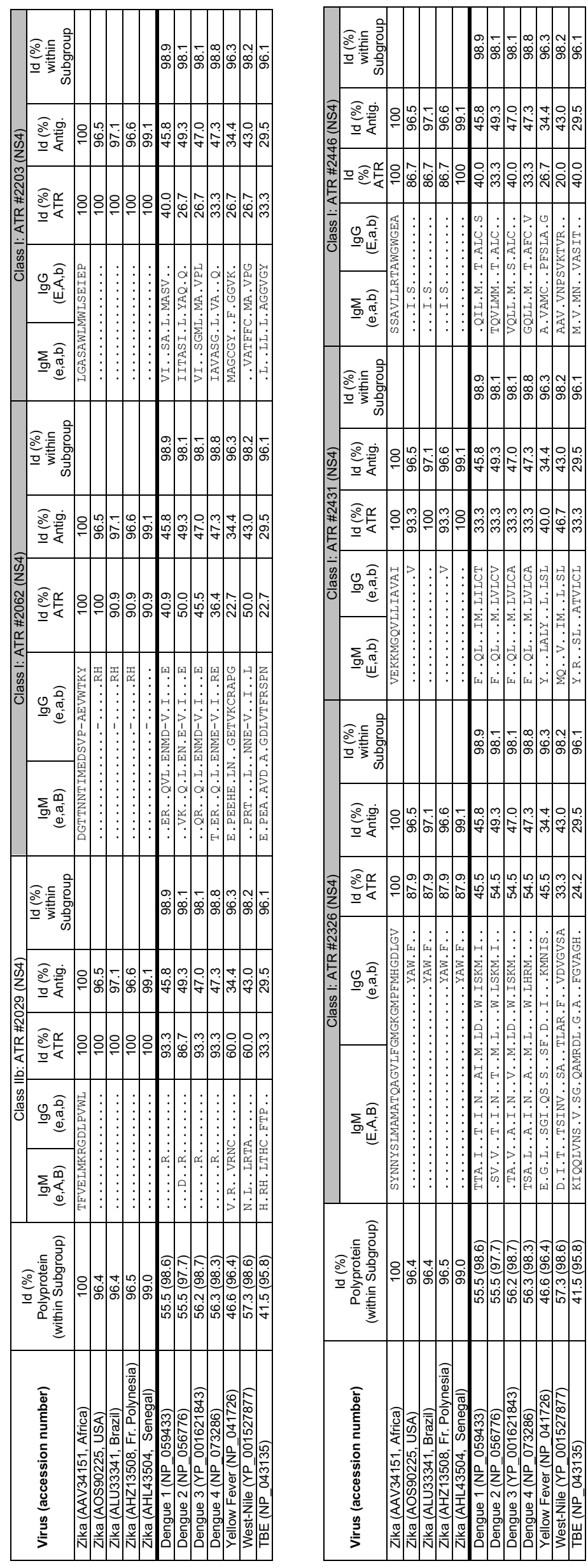

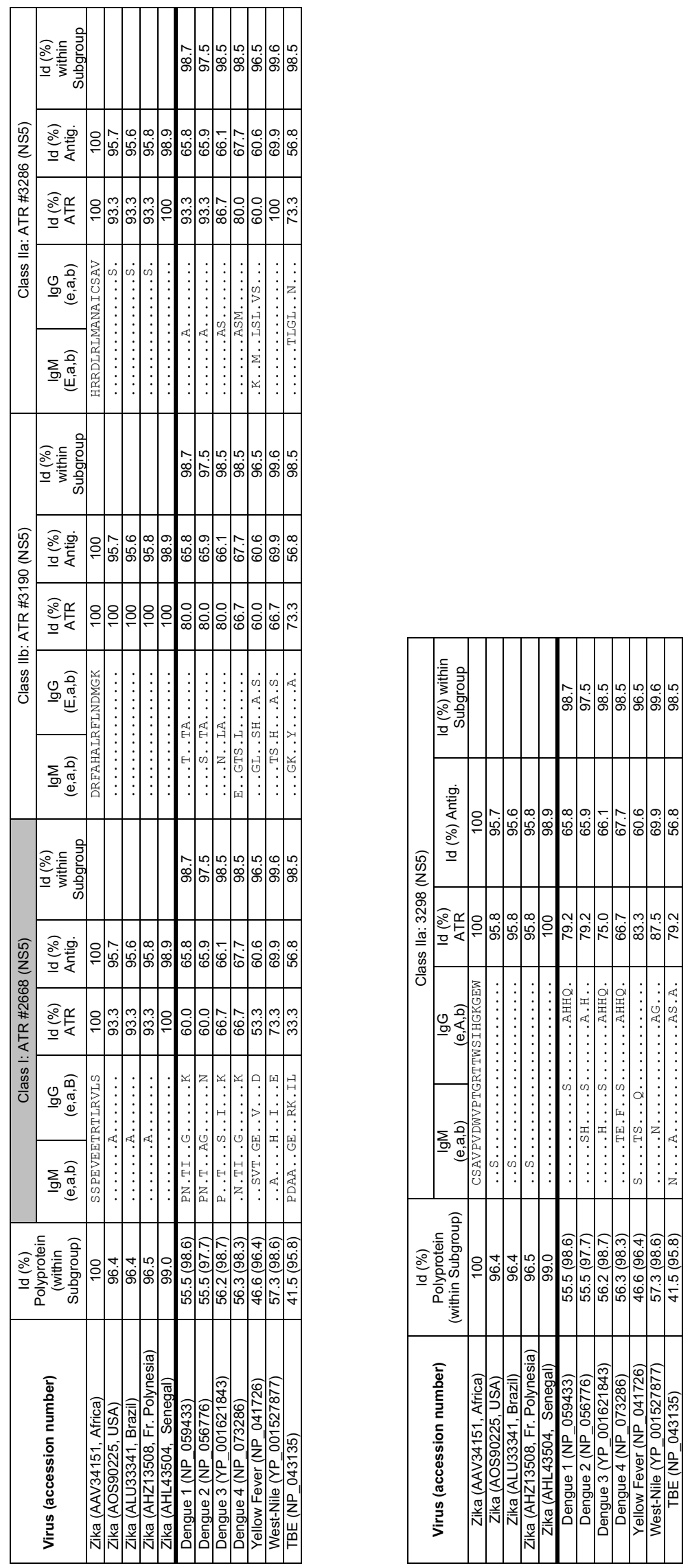

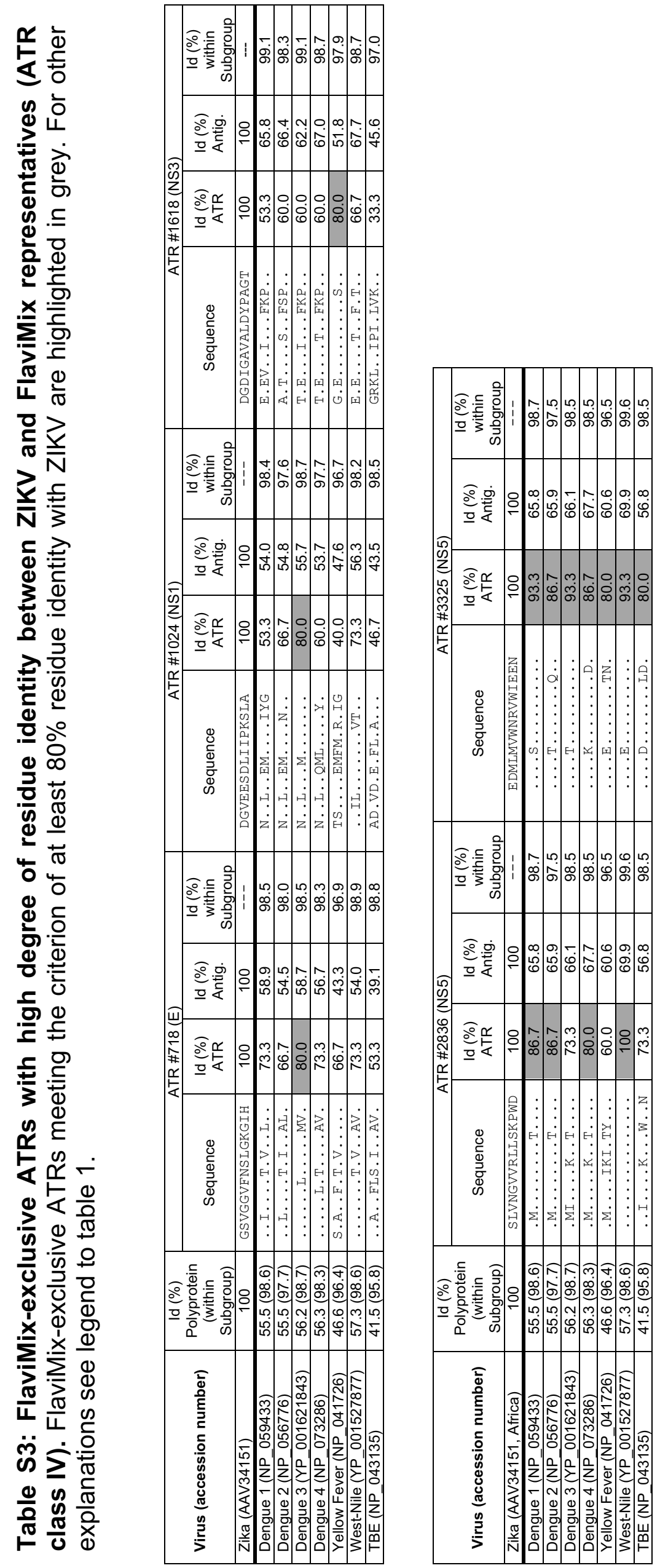
Table S4: Comparing sets of experimentally identified ATRs (this study) and of those predicted on the basis by theoretical considerations ${ }^{a, b}$

\begin{tabular}{|c|c|c|c|c|c|c|}
\hline \multirow{2}{*}{ ATR } & \multirow{2}{*}{ Sequence } & \multirow{2}{*}{ Region } & \multicolumn{2}{|c|}{ Position } & \multicolumn{2}{|c|}{ Epitope prediction } \\
\cline { 5 - 7 } & & & AMQ48981.1/ & $\begin{array}{c}\text { Xu et. al, } \\
\text { (AMQ48981.1) } \\
\text { (a) }\end{array}$ & $\begin{array}{c}\text { Homan et. al, } \\
\text { (KF383119.1) }\end{array}$ \\
\hline
\end{tabular}

ATRs identified via ZIKV microarray chips, not predicted by Xu et. al., and Homan et. al.

\begin{tabular}{|c|c|}
\hline 25 & SPFGGLKRLPAGLLL \\
\hline 361 & DSRCPTQGEAYLDKQSDTQYVCKRTLVDRG \\
\hline 799 & SKKETRCGTGVFVYN \\
\hline 817 & AWRDRYKYHPDSPRRLAAAVKQAW \\
\hline 1024 & DGVEESDLIIPKSLA \\
\hline 1177 & STSMAVLVAMILGGF \\
\hline 1213 & GGDVAHLALIAAFKVRPA \\
\hline 1282 & RAMAVPRTDNIALAILAALTPLAR \\
\hline 1309 & LVAWRAGLATCGGFMLLS \\
\hline 1441 & RLDVALDESGDFSLVEEDGPP \\
\hline 1540 & QEGVFHTMWHVTKGSALRSGEGRLDPY \\
\hline 1585 & AAWDGLSEVQLLAVPPGE \\
\hline 1618 & DGDIGAVALDYPAGT \\
\hline 1654 & IKNGSYVSAITQGKREEETPVECFEPSMLK \\
\hline 1726 & VAAEMEEALRGLPVR \\
\hline 1741 & YMTTAVNVTHSGTEI \\
\hline 1780 & YIMDEAHFTDPSSIA \\
\hline 1840 & ERAWSSGFDWVTDYSGKT \\
\hline 1870 & IAACLTKAGKRVIQL \\
\hline 1888 & TFETEFQKTK (N/H) QEWD \\
\hline 1969 & YLYGGGCAETDEDHA \\
\hline 2029 & TFVELMKRGDLPVWL \\
\hline 2062 & DGTTNNTIMEDSVPAEVWTKY \\
\hline 2095 & ARVCSDHAALKSFKE \\
\hline 2170 & IMLLGLLGTVSLGIF \\
\hline 2203 & LGASAWLMWLSEIEP \\
\hline 2308 & YAALTTFITPAVQHA \\
\hline 2326 & SYNNYSLMAMATQAGVLFGMGKGMPFYAWDFGV \\
\hline 2401 & QKRTAAGIMKNPVVDGIVVTDIDT \\
\hline 2431 & VEKKMGQVLLIAVAV \\
\hline 2446 & SSA (V/I) L (S/L) RTAWGWGEA \\
\hline 2470 & TLWEGSPNKYWNSSTATS \\
\hline 2536 & SALEFYSYKKSGITEVCREEARRALKDGVATGG \\
\hline 2611 & RKVQEVKGYTKGGPG \\
\hline 2668 & SSPEVEEARTLRVLS \\
\hline 2791 & MKIIGNRIERIRSEHAETWFFDENHPY \\
\hline 2836 & SLINGVVRLLSKPWD \\
\hline 2866 & YGQQRVFKEKVDTRV \\
\hline 2926 & LGAIFEEEKEWKTAV \\
\hline 2956 & REHHLRGECQSCVYN \\
\hline 3037 & $\begin{array}{l}\text { EEMS (R/Q) (I/A) PGG (R/K) MYADDTAGWDTRISKFDL } \\
\text { ENEAL }\end{array}$ \\
\hline 3097 & VLRPAEKGKTVMDII \\
\hline 3169 & GWDGLKRMAVSGDDCVVKP IDDRF \\
\hline 3190 & DRFAHALRFLNDMGK \\
\hline 3205 & VRKDTQEWKPSTGWD \\
\hline
\end{tabular}

\begin{tabular}{|c|c|c|}
\hline $\mathrm{C}$ & $25-39$ & $25-39$ \\
\hline$E$ & $361-390$ & $361-390$ \\
\hline NS1 & $799-813$ & $803-817$ \\
\hline NS1 & $817-840$ & $821-844$ \\
\hline NS1 & $1024-1038$ & $1028-1042$ \\
\hline NS2 & 1177-1191 & $1181-1195$ \\
\hline NS2 & $1213-1230$ & $1217-1234$ \\
\hline NS2 & $1282-1305$ & $1286-1309$ \\
\hline NS2 & $1309-1326$ & $1313-1330$ \\
\hline NS2 & $1441-1461$ & $1445-1465$ \\
\hline NS3 & $1540-1566$ & $1544-1570$ \\
\hline NS3 & $1585-1602$ & $1589-1606$ \\
\hline NS3 & $1618-1632$ & $1622-1636$ \\
\hline NS3 & $1654-1683$ & $1658-1687$ \\
\hline NS3 & $1726-1740$ & $1730-1744$ \\
\hline NS3 & $1741-1755$ & $1745-1759$ \\
\hline NS3 & $1780-1794$ & $1784-1798$ \\
\hline NS3 & $1840-1857$ & $1844-1861$ \\
\hline NS3 & $1870-1884$ & $1874-1888$ \\
\hline NS3 & 1888-1902 & $1892-1906$ \\
\hline NS4 & 1969-1983 & $1973-1987$ \\
\hline NS4 & $2029-2043$ & 2033-2047 \\
\hline NS4 & 2062-2082 & 2066-2086 \\
\hline NS4 & $2095-2109$ & $2099-2113$ \\
\hline NS4 & $2170-2184$ & $2174-2188$ \\
\hline NS4 & $2203-2217$ & $2207-2221$ \\
\hline NS4 & $2308-2322$ & $2312-2326$ \\
\hline NS4 & $2326-2358$ & $2330-2362$ \\
\hline NS4 & $2401-2424$ & $2405-2428$ \\
\hline NS4 & $2431-2445$ & $2435-2449$ \\
\hline NS4 & $2446-2460$ & $2450-2464$ \\
\hline NS4 & $2470-2487$ & $2474-2491$ \\
\hline NS5 & $2536-2568$ & $2540-2572$ \\
\hline NS5 & $2611-2625$ & $2615-2629$ \\
\hline NS5 & $2668-2682$ & $2672-2686$ \\
\hline NS5 & $2791-2817$ & $2795-282$ \\
\hline NS5 & $2836-2850$ & $2840-2854$ \\
\hline NS5 & $2866-2880$ & $2870-288$ \\
\hline NS5 & $2926-2940$ & $2930-2944$ \\
\hline NS5 & $2956-2970$ & $2960-2974$ \\
\hline NS5 & $3037-3054$ & $3041-3058$ \\
\hline NS5 & $3097-3111$ & $3101-3115$ \\
\hline NS5 & $3169-3192$ & $3173-3196$ \\
\hline NS5 & $3190-3204$ & $3194-3208$ \\
\hline NS5 & $3205-3219$ & $3209-322$ \\
\hline
\end{tabular}

\begin{tabular}{l|l}
\hline & \\
\hline
\end{tabular}




\begin{tabular}{|c|c|c|c|c|c|c|}
\hline 3229 & HHFNKL (H/Y) LKDGRS IVVPC & NS5 & $3229-3246$ & $3233-3250$ & & \\
\hline 3286 & HRRDLRLMANAICSS & NS5 & $3286-3300$ & $3290-3304$ & & \\
\hline 3298 & CSAVPVDWVPTGRTTWSIHGKGEW & NS5 & $3298-3321$ & $3302-3325$ & & \\
\hline 3325 & EDMLMVWNRVWIEEN & NS5 & $3325-3339$ & $3329-3343$ & & \\
\hline 3382 & KNTVNMVRRIIG (D/E) EEKYMDYLSTQ & NS5 & $3382-3405$ & $3386-3409$ & & \\
\hline \multicolumn{7}{|c|}{ ATRs identified via ZIKV microarray chip and predicted by $\mathrm{Xu}$ et. al., and/or Homan et. al. } \\
\hline 103 & EKKRRGADTSVGIVG & C & $103-117$ & $100-114$ & $100-110$ & $100-107$ \\
\hline 130 & $\begin{array}{l}\text { RRGSAYYMYLDRNDAISF (A/P) TTLG (V/M) NKC (H/Y) } \\
\text { (V/I) QIMDLGHMCD }\end{array}$ & prM & $130-172$ & $127-169$ & $135-139 / 143-148$ & $139-143$ \\
\hline 262 & VAIAWLLGSSTSQKV & prM & $262-276$ & $262-276$ & & $269-273$ \\
\hline 304 & $\begin{array}{l}\text { GMSGGTWVDIVLEHGGCVTVMAQDKPTVDIELVTTTVSNM } \\
\text { AEVRS }\end{array}$ & $E$ & $304-348$ & $304-348$ & $344-349$ & $305-309$ \\
\hline 388 & DRGWGNGCGLFGKGS & $\mathrm{E}$ & $388-402$ & $388-402$ & $387-427$ & \\
\hline 433 & VHGSQHSGMIVNDIGHETDENRAKVEITPNSPRAEAT & $E$ & $433-469$ & $433-469$ & $433-435 / 436-448$ & $455-459 / 464-472$ \\
\hline 499 & VHKEWFHDI PLPWHA & $E$ & $499-513$ & $503-517$ & $494-515$ & \\
\hline 526 & EALVEFKDAHAKRQT & $\mathrm{E}$ & $526-540$ & $530-544$ & $532-536$ & \\
\hline 592 & SLCTAAFTFTKVPAETLHGTVTVEVQYAGTDGPCKV & $\mathrm{E}$ & $592-627$ & $596-631$ & $608-616$ & \\
\hline 655 & TENSKMMLELDPPFG & $E$ & $655-669$ & $659-673$ & $655-666 / 670-682$ & $653-663$ \\
\hline 676 & GVGDKKITHHWHRSG & $\mathrm{E}$ & $676-690$ & $680-694$ & $670-682$ & \\
\hline 718 & GSVGGVFNSLGKGIH & $\mathrm{E}$ & $718-732$ & $722-736$ & $717-726$ & $725-729$ \\
\hline 722 & GGALNSLGKGIHQIF & $E$ & $722-735$ & $726-739$ & & $725-729$ \\
\hline 742 & LFGGMSWFSQILIGTLLMWLGLNTKNGS IS & $\mathrm{E}$ & $742-771$ & $746-775$ & $746-752 / 756-759$ & \\
\hline 898 & VNGLPHGWKAWGKSY & NS1 & $898-912$ & $902-916$ & $899-904 / 912-915$ & \\
\hline 931 & KECPLKHRAWNSFLV & NS1 & $931-945$ & $935-949$ & $932-935 / 935-938$ & \\
\hline 967 & LECDPAVIGTAVKGK & NS1 & $967-981$ & $971-985$ & $968-972$ & \\
\hline 1045 & NTREGYRTQVKGPWHSEELEIRFEECPGTKVHV & NS1 & $1045-1077$ & $1049-1081$ & $1043-1051$ & \\
\hline
\end{tabular}

\section{ATRs only predicted by $\mathrm{Xu}$ et. al., and/or Homan et. al. but not found in this study}

\begin{tabular}{|c|c|c|c|c|c|c|}
\hline / & GGFRIVNMLKR & C & $9-19$ & $9-19$ & $9-19$ & \\
\hline / & SVGKK & C & $71-75$ & $71-75$ & & $71-75$ \\
\hline / & NARK & C & $96-99$ & $96-99$ & $96-99$ & \\
\hline / & DEGVEPDDV & prM & $179-187$ & $179-187$ & $179-187$ & \\
\hline / & ARRSRRA & prM & $210-216$ & $210-216$ & & $210-216$ \\
\hline / & SHSTRKL & prM & $221-227$ & $221-227$ & & $221-227$ \\
\hline / & IGVSNRDFVEGMSGGTWVDVVL & prM & $294-315$ & $294-315$ & $294-315$ & \\
\hline / & QPENL & $E$ & $421-424$ & $421-424$ & & $421-424$ \\
\hline / & RIMLS & $E$ & $428-432$ & $428-432$ & $428-432$ & \\
\hline / & SQHSGMIVNDTG & $E$ & $436-448$ & $436-448$ & $436-448$ & \\
\hline / & ATLGG & $E$ & $464-468$ & $468-472$ & & $468-472$ \\
\hline / & AGADTG & $E$ & $513-518$ & $517-522$ & & $517-522$ \\
\hline / & GTPH & $E$ & $518-521$ & $522-525$ & $522-525$ & \\
\hline / & VVLGSQEGAVHTALAGAL & $E$ & $542-559$ & $545-562$ & $545-562$ & \\
\hline / & EMDGAKGRLS & $E$ & $562-570$ & $565-573$ & $565-573$ & $571-579$ \\
\hline / & HLKCRLKMDKL & $E$ & $574-584$ & $577-587$ & $577-587$ & \\
\hline / & PCKVPAQMAV & $E$ & $624-633$ & $628-637$ & $628-637$ & \\
\hline / & VGRLITANP & $E$ & $641-649$ & $645-653$ & $645-653$ & \\
\hline I & HQIFG & $E$ & $732-736$ & $736-740$ & $736-740$ & \\
\hline / & DVGC & NS1 & $791-794$ & $795-798$ & $795-798$ & \\
\hline I & PVPVNE & NS1 & $895-900$ & $899-904$ & $899-904$ & \\
\hline / & SYFVRAAKT & NS1 & $911-919$ & $915-923$ & $915-923$ & \\
\hline / & DTLK & NS1 & $928-931$ & $932-935$ & $932-935$ & \\
\hline / & LKVREDYSLE & NS1 & $959-968$ & $963-972$ & $963-972$ & \\
\hline
\end{tabular}




\begin{tabular}{|l|l|c|c|c|c|c}
\hline$/$ & WYGMEIRP & NS1 & $1120-1127$ & $1124-1131$ & $1124-1131$ & $1474-1482$ \\
\hline$/$ & VLMTICGMN & NS2B & $1470-1478$ & $1474-1482$ & $1487-1490$ & \\
\hline$/$ & PFAA & NS2B & $1483-1486$ & $1487-1490$ & $3130-3148$ & \\
\hline
\end{tabular}

a) Xu X, Vaughan K, Weiskopf D, Grifoni A, Diamond MS, Sette A, Peters B. 2016. Identifying Candidate Targets of Immune Responses in Zika Virus Based on Homology to Epitopes in Other Flavivirus Species. PLoS Curr 8.

b) Homan EJ, Malone RW, Darnell SJ, Bremel RD. 2016. Antibody mediated epitope mimicry in the pathogenesis of Zika virus related disease. bioRxiv doi:doi.org/10.1101/044834. 


\section{Author's contribution to the publications enclosed in this PhD thesis}

Development of Rapid Extraction Method of Mycobacterium avium subspecies paratuberculosis DNA from Bovine Stool Samples

Conceptualization, S.H., A.A.E.W., and C.-P.C.; methodology, S.H., K.F., C.-P.C., and A.A.E.W.; software, S.H.; M.R., S.B.-F., and L.M.A.A.; validation, S.H., M.R., S.B.-F., and L.M.A.A.; formal analysis, S.H., M.R., S.B.-F., and L.M.A.A.; investigation, S.H., K.F., and A.A.E.W.; resources, A.A.E.W. and C.-P.C.; data curation, S.H., M.R., and A.A.E.W.; writing original draft preparation, S.H. and A.A.E.W.; writing, review and editing, S.H., M.R., L.M.A.A., S.B.-F., K.F., C.-P.C., and A.A.E.W.; visualization, S.H., M.R., and A.A.E.W.; supervision, A.A.E.W. and C.-P.C.

Combination random isothermal amplification and nanopore sequencing for rapid identification of the causative agent of an outbreak

Conceptualization: SH and AAEW.; Data curation: SH SBF AAEW.; Formal analysis: SH SBF MW MB AAEW.; Investigation: SH MB MW AAEW.; Methodology: SH MB MW AAEW.; Resources: SH OF MF OuF AAS CPC AAEW.; Supervision: AAWE SSS AAS.; Validation: SH OF AAEW.; Visualization: SH AAWE.; Writing - original draft: SH AAEW.; Writing - review \& editing: SH OF SSS MF SBF OUF AAS MB MW CPC AAEW.

Serotyping of foot-and-mouth disease virus using oxford nanopore sequencing

S.H. and A.A.E.W. designed the study. S.H., VD., S.B.F. collected the samples and/or did laboratory work. S.H. and A.A.E.W. analysed and interpreted the data. S.H. and A.A.E.W wrote the manuscript. The manuscript was critically reviewed, edited, and approved by all authors.

Diagnosing Zika virus infection against a background of other flaviviruses: Studies in high resolution serological analysis

S.H., S.K.H., F.H., M.B., H.J.F. and A.A.E.W. designed the study. S.H., S.K.H., O.F., O.N., S.B.F., R.P., F.H., C.S.H., R.F., C.P.C., J.S.C., S.S.S., A.A.S., M.N., M.B., H.J.F. and A.A.E.W. collected the samples and/or did laboratory work. S.H., S.K.H., F.H., M.B. and A.A.E.W. analysed and interpreted the data. S.H., H.J.F. and A.A.E.W. wrote the manuscript. The manuscript was critically reviewed, edited, and approved by all authors. 


\section{List of Publications}

This thesis is based on the work conducted in the following studies:

I. Sören Hansen, Marco Roller, Lamia Alslim, Susanne Böhlken-Fascher, Kim Fechner, ClausPeter Czerny, Ahmed Abd El Wahed. Development of Rapid Extraction Method of Mycobacterium avium subspecies paratuberculosis DNA from Bovine Stool Samples. (DOI: 10.3390/diagnostics9020036)

II. Sören Hansen, Oumar Faye, Sabri S. Sanabani, Martin Faye, Susanne Böhlken-Fascher, Ousmane Faye, Amadou A. Sall, Michaël Bekaert, Manfred Weidmann, Claus-Peter Czerny and Ahmed Abd El Wahed. Combination random isothermal amplification and nanopore sequencing for rapid identification of the causative agent of an outbreak. (DOI:10.1016/j.jcv.2018.07.001)

III. Sören Hansen, Veronika Dill, Mohamed A. Shalaby, Michael Eschbaumer, Susanne Böhlken-Fascher, Bernd Hoffmann, Claus-Peter Czerny, Ahmed Abd El Wahed. Serotyping of foot-and-mouth disease virus using nanopore sequencing. (DOI:10.1016/j.jviromet.2018.10.020)

IV. Sören Hansen, Sven-Kevin Hotop, Omar Faye, Oumar Ndiaye, Susanne Böhlken-Fascher, Rodrigo Pessôa, Frank Hufert, Christiane Stahl-Hennig, Ronald Frank, Claus-Peter Czerny, Jonas Schmidt-Chanasit, Sabri S. Sanabani, Amadou A. Sall, Matthias Niedrig, Mark Brönstrup, Hans-Joachim Fritz, Ahmed Abd El Wahed. Diagnosing Zika virus infection against a background of other flaviviruses: Studies in high resolution serological analysis. (DOI:10.1038/s41598-019-40224-2)

In addition to the previous mentioned work, the following studies were also conducted during the course of this PhD work:

I. Jonas Kissenkötter, Sören Hansen, Susanne Böhlken-Fascher, Olusegun George Ademowo, Oladapo Elijah Oyinloye, Adeleye Solomon Bakarey, Gerhard Dobler, Dennis Tappe, Pranav Patel, Claus-Peter Czerny, Ahmed Abd El Wahed. Development of a panrickettsial molecular diagnostic test based on recombinase polymerase amplification assay (DOI: 10.1016/j.ab.2017.12.018). 
II. Sören Hansen, Rodrigo Pessôa, Andrezza Nascimento, Mohamed El-Tholoth, Ahmed Abd El Wahed, Sabri S. Sanabani Characterization of the microbiome composition in skin lesions caused by lumpy skin disease virus via 16s rRNA massive parallel sequencing (in preparation).

III. Sören Hansen, Abubakr Saad, Rehab Elameer, Mahmoud Elgamal, Mohamed Shalaby, Ahmed Abd El Wahed. Microbial diversity in Ancient Egyptian tomb. (in preparation).

\section{List of presentations}

I. Sören Hansen, Oumar Faye, Sabri S. Sanabani, Martin Faye, Susanne Böhlken-Fascher, Ousmane Faye, Amadou A. Sall, Michaël Bekaert, Manfred Weidmann, Claus-Peter Czerny and Ahmed Abd El Wahed. Rapid Outbreak Identification Using Point Of Need Nanopore Sequencing. (Poster) IMED 2018 Hilton Vienna, Vienna, Austria from 09-12 November 2018

II. Sören Hansen, Sven-Kevin Hotop, Oumar Faye, Oumar Ndiaye, Susanne BöhlkenFascher, Rodrigo Pessôa, Frank Hufert, Christiane Stahl-Hennig, Ronald Frank, ClausPeter Czerny, Jonas Schmidt-Chanasit, Sabri S. Sanabani, Amadou A. Sall, Matthias Niedrig, Mark Brönstrup, Hans-Joachim Fritz, Ahmed Abd El Wahed. Elucidation of specific and cross reactive Zika virus antibody target regions. (Poster) IMED 2018 Hilton Vienna, Vienna, Austria from 09-12 November 2018

III. Gayana Gunaratna, Shalindra Ranasinghe, Aresha Manamperi, Nishantha Pathirana, Hasantha KP, Renu Wickremasinghe, Nilanthi de Silva,Monica Sooriyaarachchi, Sujai Senarathne, Hasini Bannaheke, Janakie Abeynayake, Wardha Refai, Mallawarachchi $\mathrm{CH}$, Mangala Sudarshani, Dinesh Mondal, Sören Hansen, Ahmed Abd El Wahed. Rapid extraction and detection of Leishmania donovani DNA from skin lesions of suspected cases in Sri Lanka. (Poster) ASTMH 66th Annual Meeting at Baltimore Convention Center in Baltimore, MD, USA from 05-09 November 2017.

IV. George Olusegun Ademowo, Oladapo Elija Oyinloye, Sören Hansen, Muideen Kolawole Raifu, Susanne Böhlken-Fascher, Adeleye Solomon Bakarey, Claus-Peter Czerny, Ahmed Abd El Wahed. Mobile suitcase laboratory for rapid detection of Plasmodium DNA at low resource settings. (Poster) ASTMH 66th Annual Meeting at Baltimore Convention Center in Baltimore, MD, USA from 05-09 November 2017. 
V. Sören Hansen, Kim Fechner, Jenny Schäfer, Claus-Peter Cherny. Nachweis von Mycobacterium avium subsp. paratuberculosis DNA mittels eines mobilen Kofferlabors in 15 Minuten. (Oral) 36. Arbeits- und Fortbildungstagung der FG AVID „Bakteriologie“ in Kloster Banz from 13-15 September 2017.

VI. Sören Hansen, Jenny Schäfer, Ahmed Abd EI Wahed, Claus-Peter Cherny. Molecular detection of Mycobacterium avium subspecies paratuberculosis within 15 minutes. (Poster) 13. Internationale Colloquium to Paratuberculosis in Nantes, France (ICP16 Nantes), from 20-24 June 2016. 


\section{Acknowledgements}

First of all, I give my deepest thanks and greatest appreciation to Dr. Ahmed Abd El Wahed and Prof. Dr. Dr. Claus-Peter Czerny for accepting and supervising me as a PhD student at the Division of Microbiology and Animal Hygiene, University of Göttingen.

I would like to thank my colleagues at the Division of Microbiology and Animal Hygiene, especially Susanne Böhlken-Fascher, Caroline Bierschenk, Sebastian Schimkowiak, and Martin Seeländer for supporting me in my laboratory work.

A special thanks to my PhD fellows Franziska Pilger, Jonas Kissenkötter, and Henrike Ahsendorf for their accompaniment, in private and in science.

I am grateful to all co-authors of the publications this $\mathrm{PhD}$ thesis is based on, particularly to Dr. Hans Joachim Fritz and Dr. Mohamed A. Shalaby, as my scientific "Grandfathers", Dr. Kamal Eltom, Dr. Christiane Stahl-Hennig, Dr. Manfred Weidmann, Dr. Sven-Kevin Hotop, Dr. Oumar Faye, Dr. Kim Fechner, and Marco Roller for the fruitful cooperation. In addition, I would like to thank Dr. Wilhelm Wehmheuer for is great support and advise.

I can barely express my deep sense of gratitude to my family, especially to my parents and my beloved wife Marie, who has supported me during my PhD studies with love, confidence and encouragement. Thank you. 


\section{Declarations}

1. Hereby, I declare that this Ph.D. thesis has not been presented to any other examination body either in its present or a similar form.

Furthermore, I also affirm that I have not applied for a Ph.D. at any other higher school of education.

Göttingen,

(Sören Hansen)

2. Hereby, I declare that this dissertation was undertaken independently and without any unauthorised aid.

Göttingen,

(Sören Hansen) 